MICROFLUIDIC DEVICES FOR CELL BASED HIGH THROUGHPUT SCREENING 


\title{
MICROFLUIDIC DEVICES FOR CELL BASED HIGH THROUGHPUT SCREENING
}

\author{
By \\ Sarvesh Upadhyaya \\ B.Tech (Institute of Technology, Banaras Hindu University, India) \\ A Thesis \\ Submitted to the School of Graduate Studies \\ in Partial Fulfillment of the Requirements for the Degree of \\ Masters of Applied Science \\ McMaster University
}

(C) Copyright by Sarvesh Upadhyaya, April 2008. 
MASTER OF APPLIED SCIENCE (2008)

(Mechanical Engineering)
McMaster University

Hamilton, Ontario

TITLE: $\quad$ Microfluidic Devices for Cell Based High Throughput Screening

AUTHOR: $\quad$ Sarvesh Upadhyaya,

B.Tech (Institute of Technology, Banaras Hindu University, India)

SUPERVISOR: Dr. Ravi Selvaganapathy, Asst. Professor (Mechanical Engineering, McMaster University)

NUMBER OF PAGES: $\quad$ XVII, 135 


\section{ABSTRACT}

Cell based High Throughput Screening (HTS) has become a very important method in pharmaceutical drug discovery and presently carried out using robots and well plates. A microfluidics based device for cell based HTS using traditional cell culture protocol would be a significant addition to the field. In this thesis novel microfluidic HTS devices targeted for cell based assays having traditional non-compartmentalized agar gel as cell culture medium and electric control over drug dose is being reported.

The basic design of device consists of a gel layer supported by a nanoporous membrane that is bonded to microchannels underneath it. The pores of membrane are blocked everywhere except in selected regions that serve as fluidic interfaces between the microchannel below and the gel above. Upon application of electric field nanopores start to act as electrokinetic pumps. By selectively switching an array of such micropumps, a number of spots - containing drug molecules - are created simultaneously in the gel layer. By diffusion drugs reach to the top surface of gel where cells are to be grown. Based on this principle, a number of different devices are fabricated using microfabrication technology. The fabricated devices include, single drug spot forming device, multiple drug spot forming device and microarray of drug spots forming device.

By controlling pumping potential and duration spots sizes ranging from $200 \mu \mathrm{m}$ to $6 \mathrm{~mm}$ diameter and having inter-spot distances of $0.4 \mathrm{~mm}-10 \mathrm{~mm}$ have been created. Absence of diffusional transport through the nanoporous interfaces without electric field 
Master's Thesis - Sarvesh Upadhyaya - McMaster University - Mechanical Engineering

is demonstrated. A number of representative molecules, including surrogate drug molecules (trypan blue, and methylene blue) and biomolecules (DNA and protein) were selected for demonstration purpose. Dosing range of 50-3000 $\mu \mathrm{g}$ and spot density of 156 spots $/ \mathrm{cm}^{2}$ were achieved.

The drug spot density was found to be limited by molecular diffusion in gel and hence numerical study was carried to find out ways for density increase. Based on this simulation, a method for diffusion reduction called diffusion barrier was proposed. Diffusion barrier used specially dimensioned (having shallow grooves) gel sheet to reduce the diffusion. 


\section{ACKNOWLEDGEMENTS}

Leaving one's country of birth and going abroad for higher studies has not been as easy as it sounded to me. And now when I am nearing the finish line, I deeply feel that it would not have been at all possible without help, support, love and guidance of several people.

I would like to thank my advisor, Dr. Ravi Selvaganapathy - a fine professional and a nice person - for his constant support and guidance. I have found his way of supervising - supportive and encouraging to think and act- very useful for inculcating a habit of independent thinking, through analysis, risk taking and 'do it' attitude. His saying "Sarvesh, why don't you try this as well" would remain in my memory always. Without him it was impossible to complete the work and gain the learning which I have during my last two years.

I would also like to thank my lab friends, Bala, Arash, Geetha, Pouya and Wen. They have been of immense help in setting up my experiments and their quick tips were very useful. They have been wonderful colleagues and fun to work with. Support provided by Dorris and Zhillin (Engineering Physics) in microfabrication was very useful for timely completion of my work.

My parents, brother Somesh and close friend Sudha, the finest people I have ever known, undoubtedly gained my highest respect and appreciation when they allowed me to come to Canada bearing all the pain of sending me tens of thousands of mile 
Master's Thesis - Sarvesh Upadhyaya - McMaster University - Mechanical Engineering aways. They were always there when I needed them, to encourage me and to support me. I thank them all by my way of life and by becoming a better person and professional. 


\section{TABLE OF CONTENTS}

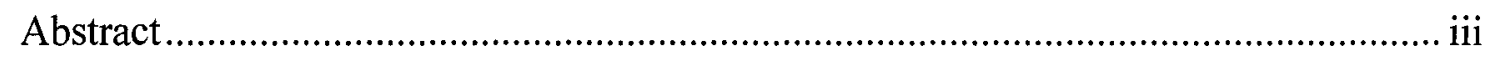

Acknowledgements........................................................................................

Table of Contents........................................................................................ vii

List of Figures .......................................................................................

List of Tables ........................................................................................... Xvii

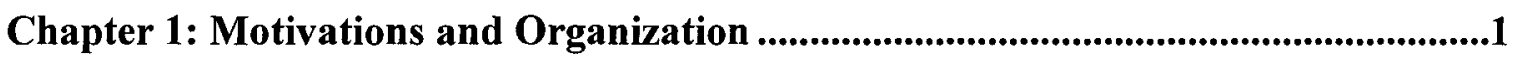

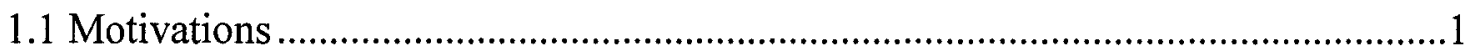

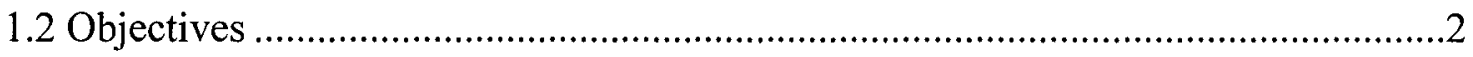

1.3 Thesis Organization ..................................................................................2

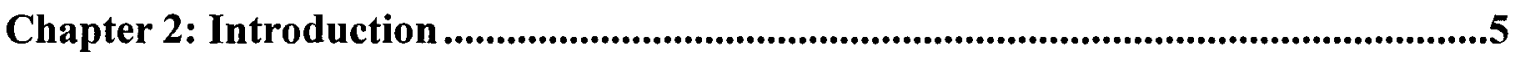

2.1 Introduction to Microfluidics......................................................................5

2.2 Microfluidics in Biological Studies ..........................................................6

2.3 Drug Discovery Process ..............................................................................

2.4 High Throughput Screening (HTS) ............................................................ 12

2.5 Cell Based HTS ..................................................................................... 14 
Master's Thesis - Sarvesh Upadhyaya - McMaster University - Mechanical Engineering

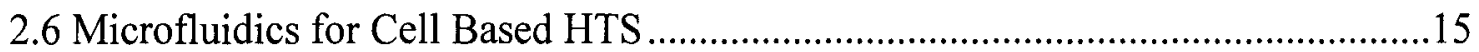

Chapter 3: Microfluidic Devices for Cell Based HTS .....................................................17

3.1 Previous Works for Cell Culture Based HTS ……...............................................18

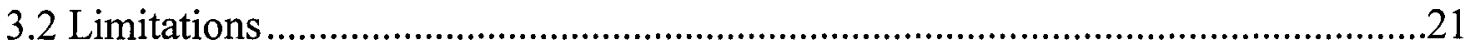

3.2.1 Downsizing of Macro World Approaches .........................................................21

3.2.2 Use of Non-Traditional Cell Culture Materials ........................................................22

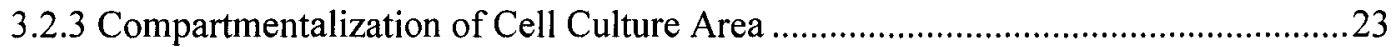

Chapter 4: Nanoporous Membrane Microfluidic Device for Cell Based HTS ...........25

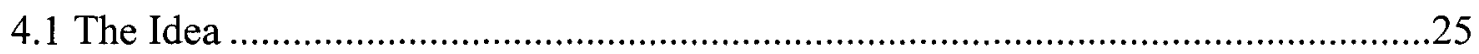

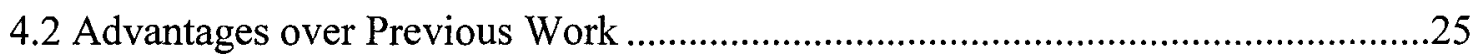

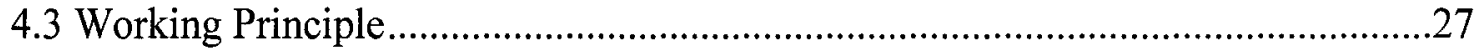

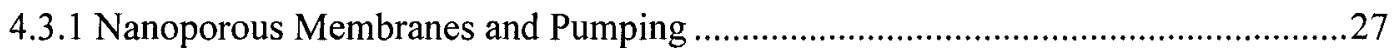

4.3.1.1 Electroosmotic Pumping .................................................................28

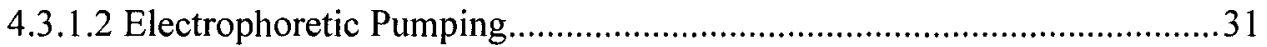

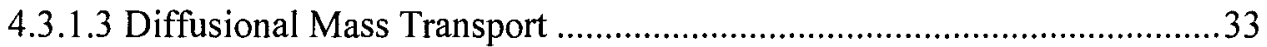

4.3.1.4 Relative Magnitude ff Various Pumping Modes ....................................34

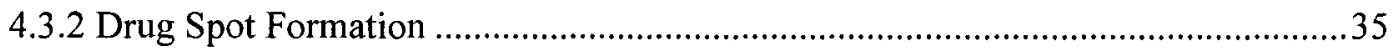


Master's Thesis - Sarvesh Upadhyaya - McMaster University - Mechanical Engineering

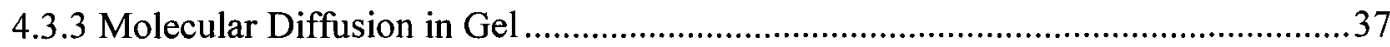

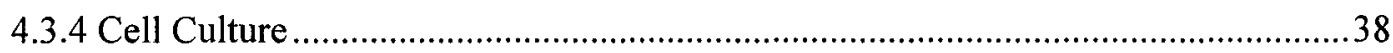

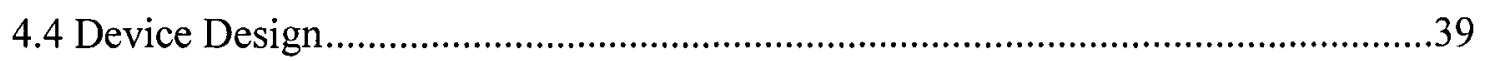

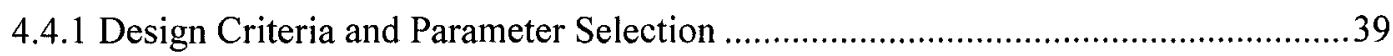

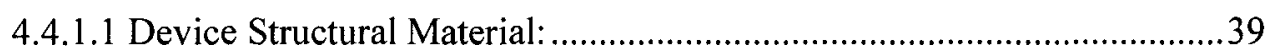

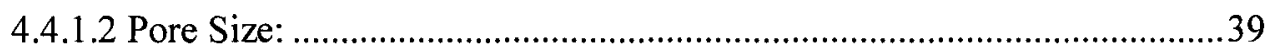

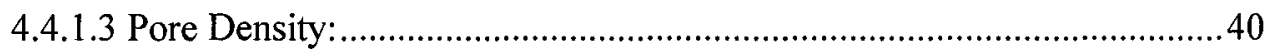

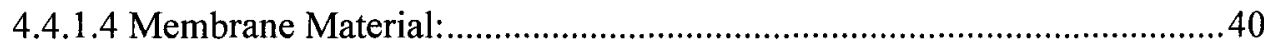

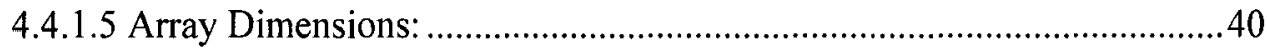

4.4.1.6 Gel Material And Dimensions: ...................................................... 40

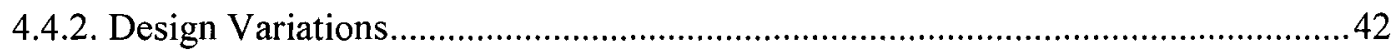

Chapter 5: Fabrication and Experimental Setup .........................................................43

5.1 Fabrication Materials .......................................................................................43

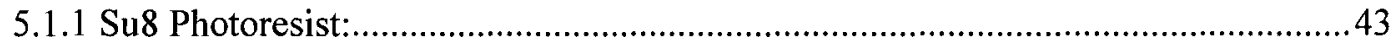

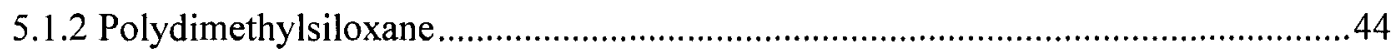

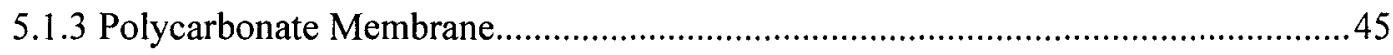

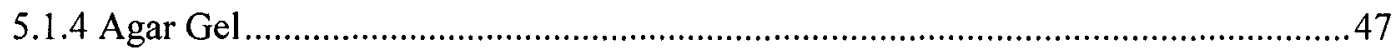


Master's Thesis - Sarvesh Upadhyaya - McMaster University - Mechanical Engineering

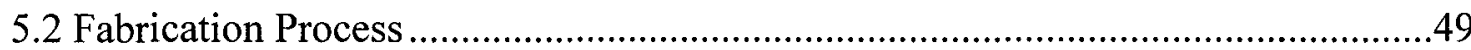

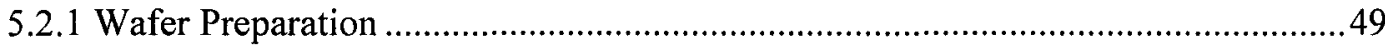

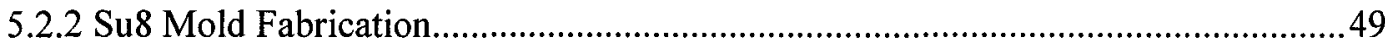

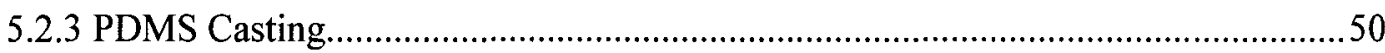

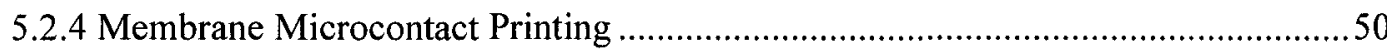

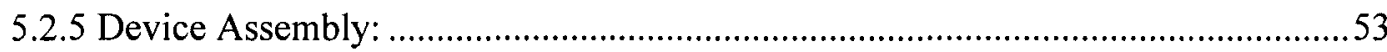

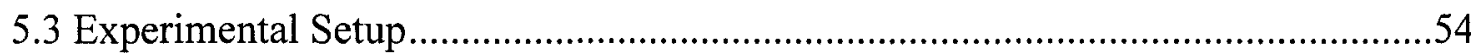

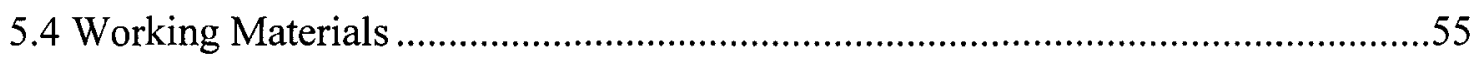

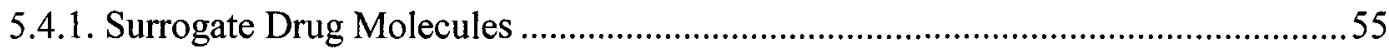

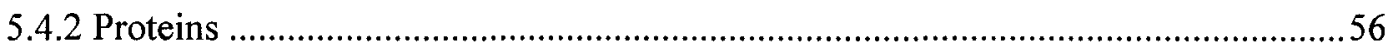

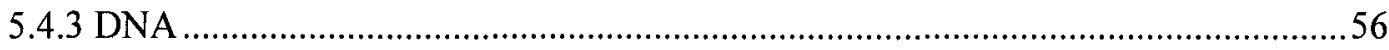

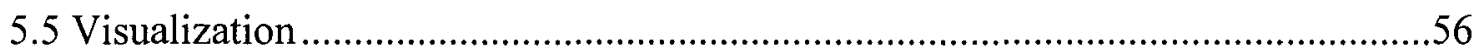

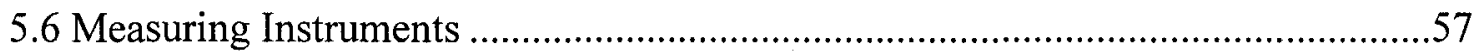

Chapter 6: Single Drug Spot Devices......................................................................59

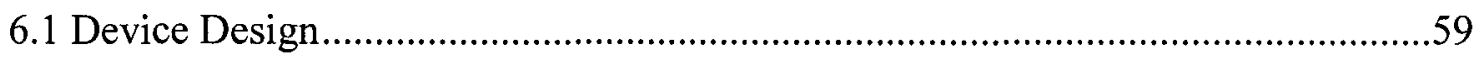

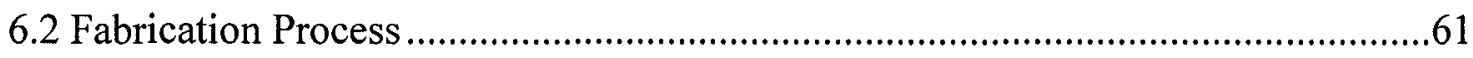

6.3 Fabricated Devices and Experimental Setup ..............................................62 
Master's Thesis - Sarvesh Upadhyaya - McMaster University - Mechanical Engineering

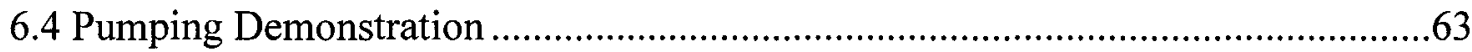

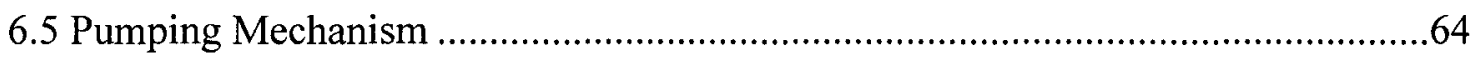

6.6 Post Pumping Drug Diffusion .........................................................................65

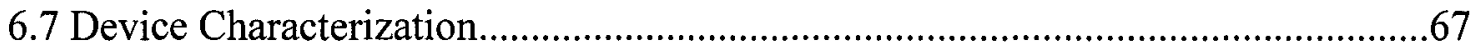

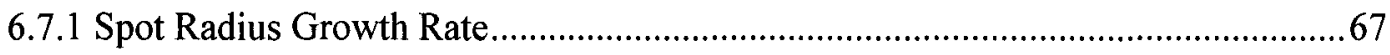

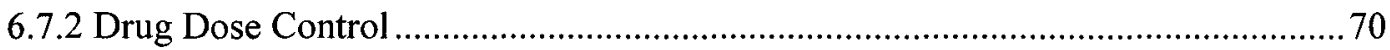

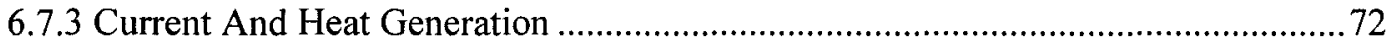

Chapter 7: Multiple Drug Spot Devices...........................................................................................74

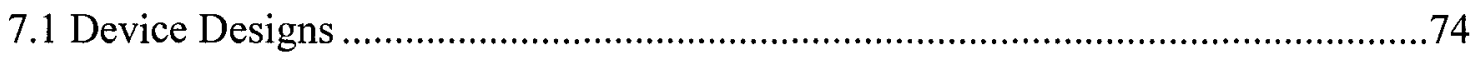

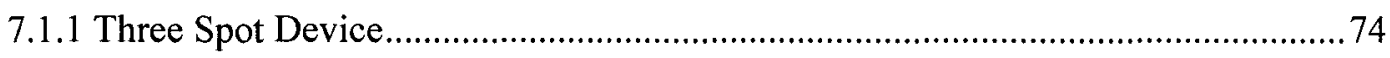

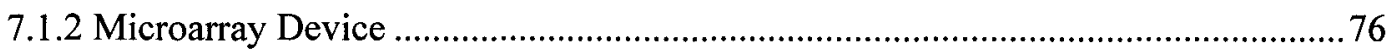

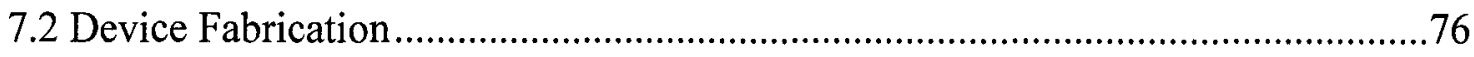

7.3 Creation of Independent Drug Spots ....................................................................

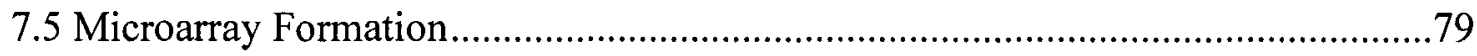

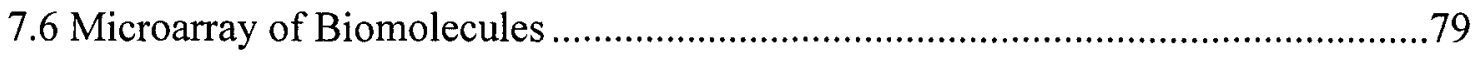

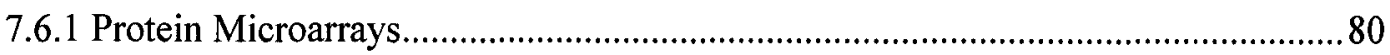

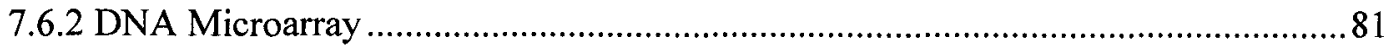


Master's Thesis - Sarvesh Upadhyaya - McMaster University - Mechanical Engineering

7.6 Spot-Spot Interaction due to Diffusion

Chapter 8: Molecular Diffussion Barrier For Gel Slab .84

7.1 The Physics of the Problem .84

7.2. Diffusional Mass Transport in Gel Slabs having Different Thicknesses. .86

7.2.1 The Problem Definition .87

7.2.1 Numerical Simulation In Comsol Multiphysics

7.2.3 Comparison Of Concentration Distribution .88

7.2.4 Comparison of Mass Transport .88

7.3 Diffusion Barrier in Gel Slab .92

7.3.1 Comparison with Simple Gel Slab .92

Chapter 9: Contributions and Future Work .96

9.1 Contributions .96

9.1.1 A Novel Idea for Cell Based HTS. .96

9.1.2 Design Fabrication and Testing of Microfluidic HTS Devices .97

9.1.3 Diffusion Barrier .97

9.2 Future Work .98 
Master's Thesis - Sarvesh Upadhyaya - McMaster University - Mechanical Engineering

Appendixes

Appendix 1: Su8 Mold Frabricataion

117

Appendix 2: PDMS Microcontact Printing

Appendix 3: Device Assmebly Process

Appendix 4: Visualization of Protein

Appendix 5: Visualization of DNA

Appendix 6: Microchem Corp. Su8-100 Data Sheet 126

6.1 Su-8 Spinning Speed vs. Film Thickness 126

6.2 Su-8 Film Thickness vs. Exposure Energy

Appendix 7: Polycarbonate Membrane Data Sheet 128

7.1 Product Characteristics 128

7.2 Performance Characteristics. 129

Appendix 8: Bovine Serum Albumin Properties Supplier Data Sheet 131 


\section{LIST OF FIGURES}

Figure 2.1 Schematic representation of drug discovery.

Figure 2.2 Drug discovery costs. .11

Figure 3.1 Schematic of continuous perfusion device for cell culture: .18

Figure 3.2 Schematic of Caliper Life Sciences microfluidic HTS device:........................19

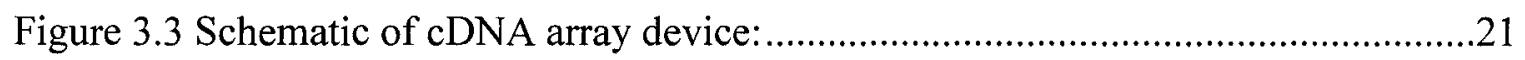

Figure 4.1 Schematic diagram of the approach for cell based HTS: ................................26

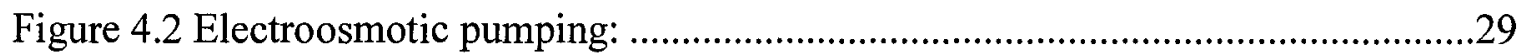

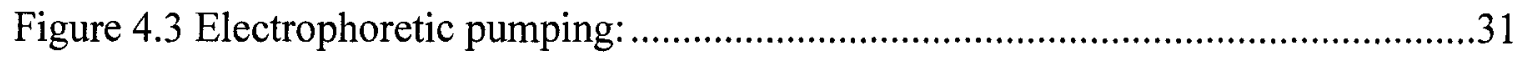

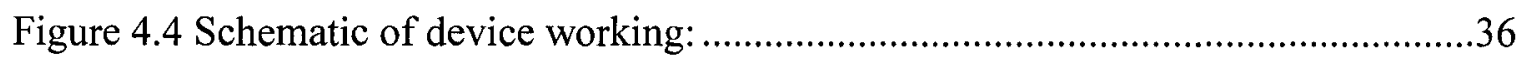

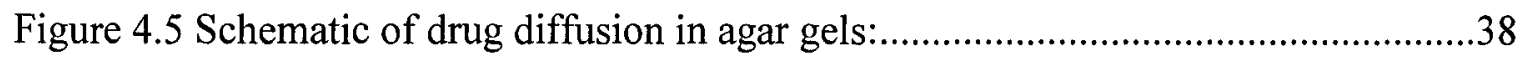

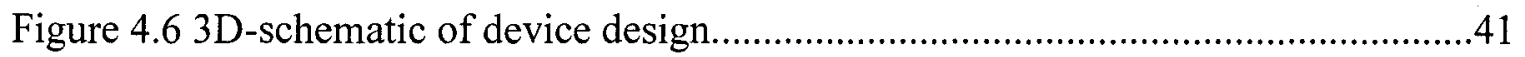

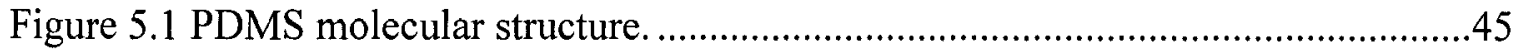

Figure 5.2. SEM image of nanoporous membrane: ....................................................46

Figure 5.3. General molecular structure of agar backbone:.............................................48

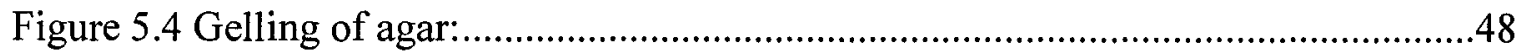

Figure 5.5 An example of PDMS sheet used for microcontact printing: ........................50

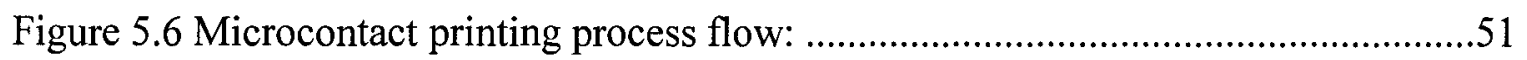

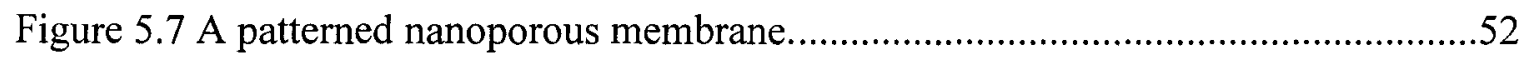

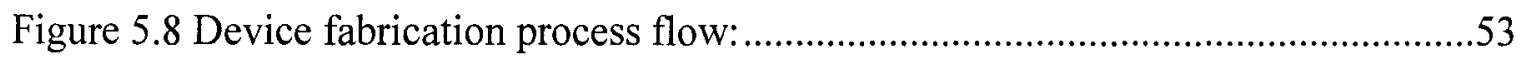

Figure 5.9 Schematic of general experimental setup.................................................5 
Master's Thesis - Sarvesh Upadhyaya - McMaster University - Mechanical Engineering

Figure 5.10 Calibration curve for trypan blue for photometric measurements:

Figure 6.1 Single spot forming device design:

Figure 6.2 One of the single spot devices:

Figure 6.3 Single spot forming device experimental setup:

Figure 6.4 Drug pumping demonstration:

Figure 6.5 Post pumping drug diffusional behavior (qualitative): .66

Figure 6.6 Post pumping drug diffusional behavior (quantitative):

Figure 6.7 Drug spot radius growth during pumping (qualitative): .68

Figure 6.8 Drug spot radius growth during pumping (quantitative):

Figure 6.9 Drug spot radius growth after pumping (quantitative): 70

Figure 6.10 Drug dose vs. time: .71

Figure 7.1 Three spot forming device design: 75

Figure 7.2 Microarray forming device design: .77

Figure 7.3 Fabrication process flow for microarray forming device design. .78

Figure 7.4 Independent spot creation.

Figure 7.5 Microarray of drug spots: .80

Figure 7.6 Microarray of protein spots. .81

Figure 7.7 Microarray of DNA spots. .82

Figure 7.8 Mixing of drug spots. .82

Figure 8.1 Average pore size vs. gel concentration for Agar gel. 85

Figure 8.2 Gel slabs for modeling. .86

Figure 8.3 Comparison of concentration distribution. .89 
Master's Thesis - Sarvesh Upadhyaya - McMaster University - Mechanical Engineering

Figure 8.4 Concentration profile comparison of thin and thick gel................................90

Figure 8.5 Concentration in $1 \mathrm{~mm}$ thick gel slab with diffusion barrier. ..........................91

Figure 8.6 Concentration profile comparison of gel with and without diffusion barrier: .93

Figure 8.7 Concentration profile comparison of gel with and without diffusion barrier: .94 
Master's Thesis - Sarvesh Upadhyaya - McMaster University - Mechanical Engineering

\section{LIST OF TABLES}

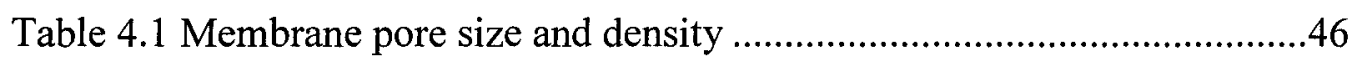

Table 6.1: Current and heat generation in a typical operating condition...........72 
Master's Thesis - Sarvesh Upadhyaya - McMaster University - Mechanical Engineering

\section{CHAPTER 1}

\section{MOTIVATIONS AND ORGANIZATION}

\subsection{MOTIVATIONS}

In recent years, cell based high throughput screening (HTS) has become a very important method in pharmaceutical drug discovery [Sundberg 2000, Khandurina 2002]. In HTS a large number $(100,000-1,000,000)$ of chemical compounds are tested against some selection criteria (e.g. tendency to bind to a protein) to select starting compounds for drug development. These compounds are further screened, modified and optimized to make a drug. Presently, in order to test such large number of compounds, HTS is performed using well plates and robotic arms. Well plates (typically a plastic plate having large number of wells in it) enables performing a number of experiments per plate and robotic arms are used for automated chemical and well plate handling [Drews 2000]. However, the main drawbacks of this approach are the high associated costs due to high volumetric throughputs [Dickson 2004]. Microfluidics, which encompasses fluid handling at smaller lengths and volumes, offers a number of advantages for HTS applications including higher number of assays per plate and lower reagent consumptions [Whitesides 2004]. As compared to other types of HTS (e.g. enzyme based), cell based HTS (a type of HTS which uses biological cells for testing) has got a number of unique advantages. Cell based HTS generate data which is better representation of human body 
Master's Thesis - Sarvesh Upadhyaya - McMaster University - Mechanical Engineering

and have greater physiological significance. The leads obtained have better probability to become a drug [Khandurina 2002]. A microfluidics based device for cell based HTS using traditional cell culture protocols and precise control of drug supplies would be a significant addition to the field. These developments are expected to significantly reduce costs in the current 11.3 billion USD HTS market [Gardner 2004].

\subsection{OBJECTIVES}

The objective of this thesis is to develop a microfluidics based method for cell based HTS. The objective is sub-divided into the following subobjectives:

1. Identification of a suitable method to supply drugs in a microarray format for cell culture in a gel medium.

2. Design, fabrication and test of device/devices; demonstration and characterization of device suitability for its intended application.

3. Execution of performance maximization studies including numerical simulations of the physical phenomena.

\subsection{THESIS ORGANIZATION}

The chapters in this thesis are organized as following:

Chapter 2 outlines an introduction to microfluidics followed by a discussion of contemporary drug discovery process and HTS followed by an introduction to cell culture based HTS. The relevance and application of microfluidics for HTS is also covered. 
Master's Thesis - Sarvesh Upadhyaya - McMaster University - Mechanical Engineering

In chapter 3, the current state of the art in cell based HTS is covered.

In chapter 4 the working principle of the device will be analyzed and discussed. Salient topics include nanoporous membranes and electrokinetic pumping behavior, drug spot formation, microarray formation and drug diffusion in gels. Also, the basic design of various devices is discussed. The details of individual device design are covered in their respective chapters.

Chapter 5 deals with device fabrication. The fabrication materials and process flows for fabrication of devices are discussed. The specific details regarding fabrication of individual devices are discussed in their respective chapters. Experimental setup, model drugs and measurement instrumentation are also discussed in this chapter.

Chapter 6 describes the first device, a single drug spot forming device. The design, fabrication process and testing results are covered. Device characterization, many of which are also applicable for other devices, is included in this chapter.

Chapter 7 also deals with an individual device but this time, a multi drug spot forming device. It covers design, fabrication, test results and the ability to work with biomolecules. This chapter also covers the number of spots that can be created per unit area (spot density), the desire to maximize this density and the limiting effects of extraneous diffusive processes.

Chapter 8 starts with the objective to analyze in detail the problem of drug diffusion which includes a numerical model to study drug diffusion behavior. Based on 
Master's Thesis - Sarvesh Upadhyaya - McMaster University - Mechanical Engineering

this numerical study, a method for the mitigation of diffusion (called diffusion barrier) is proposed.

Chapter 9 covers the main contributions of this thesis which includes an idea to accomplish cell culture based HTS in a traditional culture media without compartmentalization. This chapter ends with proposed future works. 
Master's Thesis - Sarvesh Upadhyaya - McMaster University - Mechanical Engineering

\section{CHAPTER 2}

\section{INTRODUCTION}

\subsection{INTRODUCTION TO MICROFLUIDICS}

Microfluidics describes the field of fluid handling at small length scales and volumes. Generally microfluidic devices are made by microfabrication technology with sub-millimeter size features with total device dimensions on the order of several millimeters. These devices work with volumes ranging from picoliters to milliliters. Although the popularity of the field has increased in last two decades [Whitesides 2006], the field itself is relatively older. For example, during the late 1970s, a microfluidic based chromatograph was developed at Stanford University [Terry 1979] while IBM developed ink jet micronozzles [Bassous 1977] for printing applications. The field remained mostly stagnant for a decade, but had transformed into a rapidly developing research area by the 1990s [Gravensen 1993]. Most of the early device incarnations, such as capillary electrophoresis devices, micropumps [Smits 1990, Laser 2004], microvalves [Yanagisawa 1993, Oh 2006] and micromixers [Miyake 1993, Nguyen 2005], were not application driven but were developed to demonstrate feasibilities [Whitesides 2006]. However, recently this trend has shifted to applications-based design of devices with an associated larger number of complete or near complete microfluidic devices being reported [Whitesides 2006]. For example, a complete microfluidic device for finding out 
Master's Thesis - Sarvesh Upadhyaya - McMaster University - Mechanical Engineering

the optimum conditions for protein crystallization by optimizing a number of variables (such as $\mathrm{pH}$, ionic strength and composition, co-solvents, and concentration) has been developed by Zheng et al. [Zheng 2004]. Lee et. al. [Lee 2005b] made a microfluidic device capable of synthesizing radioactive compound $\left({ }^{18} \mathrm{~F}\right)$ for positron emission tomography. A number of microfluidic devices useful in biological studies like high throughput screening [Pihl 2005, Dittrich 2006] and bioanalysis [Whitesides 2006] have also been developed.

\subsection{MICROFLUIDICS IN BIOLOGICAL STUDIES}

Microfluidics in biological cell based studies such as cell cytometry [Li 1997, Fielder 1998], cellular biosensors [Bousse 1996, Vo-Dinh 2001] and cell culture [Inoue 2001], has become one of the driving forces for the proliferation of the microfluidics field [Whitesides 2006]. The main advantages of microfluidics for cell based applications can be summarized as follows:

- Typical dimension of biological cells, which ranges from few microns to $100 \mu \mathrm{m}$, are in excellent dimensional agreement with typical microchannel dimensions used in microfluidics (few $\mu \mathrm{m}$ to few hundred $\mu \mathrm{m}$ ) making microfluidic devices ideally suitable for cell based studies [Andersson 2003].

- Interest in viable biological cell based studies has rapidly increased [Andersson 2003]. 
Master's Thesis - Sarvesh Upadhyaya - McMaster University - Mechanical Engineering

- The potential to integrate a number of analysis steps on a single chip [Andersson 2003].

- The relative magnitudes of various forces change as dimensional order of the device shifts from the macroscale to the microscale [Trimmer 1996, Wautelet 2001]. For example, according to the scaling laws [Trimmer 1996, Wautelet 2001], surface tension and Van der Waals forces scale linearly with length, gravitational and magnetic forces scale to the cube of the length dimension. Therefore, gravity and magnetic forces dominate in the macro level world but are not so significant in the micro world. Another example of scaling is the high electric fields that can be generated by using lower voltages.

One good use of such scaling is the lower thermal time constants that can be achieved for Polymerase Chain Reaction (PCR) chambers by using smaller heat capacity (i.e. smaller mass) and faster heat transfer rates (larger surface area). Smaller thermal time constant allows faster PCR thermal cycle, thereby reducing the overall time required for the PCR [Giordano 2001]. Using microfluidics Taylor et. al. reported a 5 fold reduction in time requirement as compared to convention PCR [Taylor 1997]. Another example is the high electric fields that can be generated at microscale without requiring dangerously high voltages for electrophoretic systems, which leads to decreased separation times [Koutny 1996]. 
Master's Thesis - Sarvesh Upadhyaya - McMaster University - Mechanical Engineering

- By virtue of its ability of manipulating microparticles and forces, microfluidics also offers the ability to simulate cell-substrate interactions vital for cell functioning [Folch 2000, Takayama 2001].

- Fabrication technology of microfluidics is very closely related with microelectronic fabrication methods. This enables high volume parallel fabrication of large number of devices leading to lower costs associated with batch manufacturing.

The above discussion illustrates how microfluidics offer performance advantages in the context of cell based biological studies. Due to these reasons, microfluidics has been used in a number of techniques dealing with biological cells, for example cell cytometry, i.e. to count cells [Fu 1999, Beebe 2000], cellular biosensors i.e. using cells as sensors [Bousse 1996 Pancrazio 1999], cell chemotaxis i.e. migration of cells in response to presence, absence or gradient of chemicals [Sinclair 2002], and cell culture [Inoue 2001, Park 2003]. The drug discovery process (which extensively uses biological cells) used by pharmaceutical companies is one of the fields where microfluidics is expected to have enormous potential [Dittrich 2005].

\subsection{DRUG DISCOVERY PROCESS}

Drug discovery processes can be defined as a collection of methods and approaches used to identify new compounds that are useful in the treatment of diseases. In the last few decades, the drug discovery process has gone through an evolutionary process [Drews 2000]. One of the major issues with the present drug discovery process is 
Master's Thesis - Sarvesh Upadhyaya - McMaster University - Mechanical Engineering

that it has become extremely time consuming and cost intensive [Dickson 2004]. The typical lead time to fully develop a drug can be as high as a decade with development costs easily reaching the several hundred million dollars mark [Dickson 2004]. Presently, a typical drug discovery process can be shown by Figure 2.1 . The process is fairly lengthy and is divided into sub-stages, each represented by an arrow in the figure [Dickson 2004].

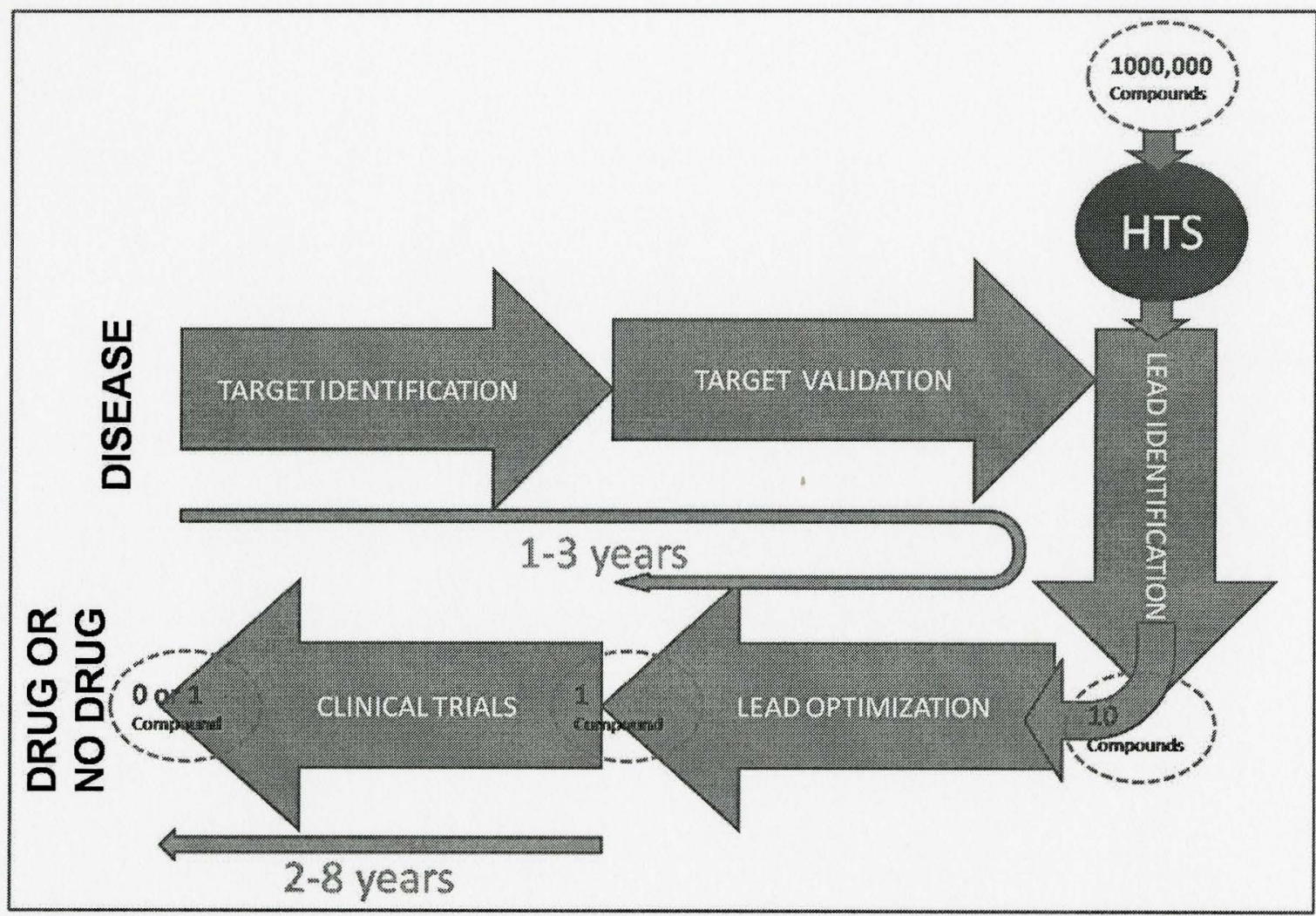

Figure 2.1 Schematic representation of drug discovery. Each stage may take several years to complete. The latter stages, i.e. clinical trials, generally are the costliest. Every effort is made to have good results in earlier stages in order to avoid failures in latter stages. HTS is used for lead identification IDickson 20047. 
Master's Thesis - Sarvesh Upadhyaya - McMaster University - Mechanical Engineering

The discovery process starts with target identification, i.e. to find out suitable biomolecules having critical role in disease progress and which is drugable (i.e. can be interfered with, without adverse effects). After target identification the second stage is target validation. Target validation is the process in which it is demonstrated that the interfering of target molecule functioning can lead to desirable changes in disease behavior. The target validation involves studies in living animals as well as in cell-based models. Market and business potential of the targets are also critically evaluated at this stage.

Lead identification stage (the third stage, i.e. after target validation) is defined as the first step of finding chemical compounds that can be further modified and optimized to become a drug. High Throughput Screening (HTS) is a vital technology used in lead identification. In HTS, a large number of chemical compounds (also popularly known as chemical library) are tested against an established testing method to short list compounds. Details about HTS are given in next section. A typical compound library has some $\sim 1$ million compounds and at the end of lead identification phase typically few tens of them are selected. Lead optimization phase, which comes after lead identification, deals with further optimizing the chemical structures of the leads for enhancing the desirable properties of the leads. During lead optimization phase molecular modeling, knowledge of synthetic chemistry, and empirical knowledge of structure-function relationships of known compounds are used to discover and design new drugs. During this phase absorption, distribution, metabolism, and excretion (ADME) of drugs is also assessed. During all these phases, collectively called as pre-clinical trials, complete study of drugs 
Master's Thesis - Sarvesh Upadhyaya - McMaster University - Mechanical Engineering

on two sample animals are also done [Drews 2000]. At the end of this process typically a single molecule is chosen as candidate drug and applications are made to governmental authorities to get approval for clinical trials. A drug may not be allowed for clinical trials due to a number of reasons including safety of human patients during clinical trials. As per a study average total costs per drug before clinical trials in 2001 was 305 million US\$ [DiMasi 2003]. This value is significantly larger than that of year 1991, when per drug average preclinical cost was 214 million US\$ [DiMasi 1991].

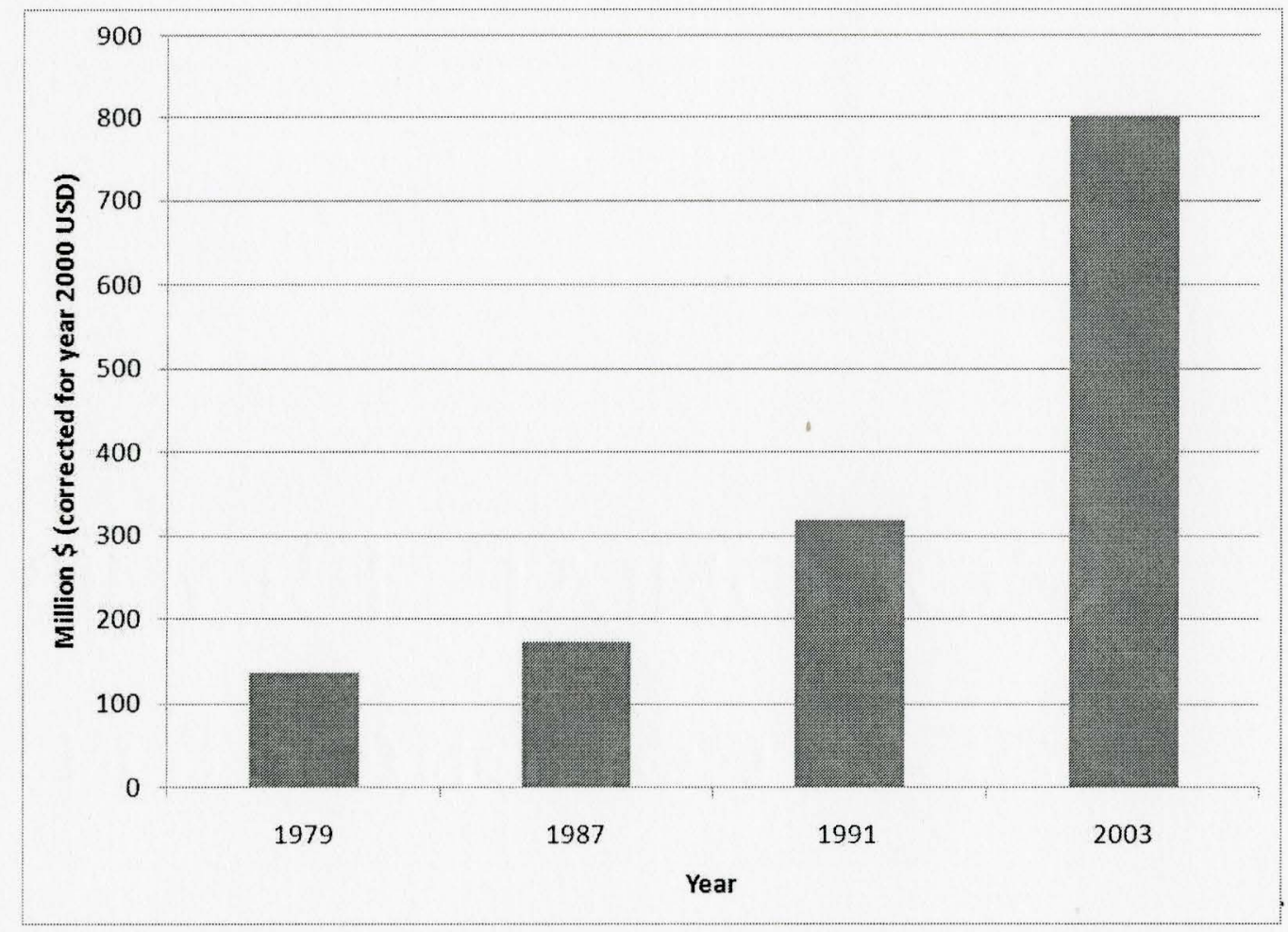

Figure 2.2 Drug discovery costs. The bars represent the average amount that was spent to develop a single drug. There has been a strong growth in costs which is expected to continue in the future [Dickson 2004].

Clinical trials are the most expensive and time consuming process of drug discovery, typical times range from 2-8 years with average being 5 years. The rejection of 
Master's Thesis - Sarvesh Upadhyaya - McMaster University - Mechanical Engineering

compound at this stage is disastrous, as money spend at earlier stages are also wasted. Unfortunately, as per a study, $90 \%$ of the compounds that go through clinical trials are rejected [Dickson 2004]. Figure 2.2 shows the increasing trend in costs for developing a single drug [Dickson 2004]. There are several drivers for this increasing cost trend. For example: high-risk, poorly validated targets, huge compound libraries with low quality molecules, tougher governmental regulations and poor risk management by pharmaceutical companies [Booth 2004]. The high (and increasing) costs put pressure on preclinical trials to economically generate better quality candidate drugs that do not fail during clinical trials. This pressure percolates down to HTS stage to generate better leads in an economical and timely manner.

The higher costs ultimately result in a lesser number of drugs being developed as well as a higher cost of drugs for patients. High throughput screening (HTS), a technology used for lead identification in drug discovery, also especially relevant to the present work, is described herein.

\subsection{HIGH THROUGHPUT SCREENING (HTS)}

Out of several stages of drug discovery, as shown in Figure 2.1, HTS is a technology useful in lead identification [Huser 2007, Hertzberg 2000]. Lead identification can be defined as the first step of finding chemical compounds that can be further modified and optimized to become a drug. In HTS, a large number of chemical coumpounds (also popularly known as chemical libraries) are tested against an established testing method. A typical compound library can have $\sim 10^{4}$ to $10^{6}$ compounds 
Master's Thesis - Sarvesh Upadhyaya - McMaster University - Mechanical Engineering

Hertzberg 2000]. Based on a certain selection criteria (e.g. binding to certain protein), a very small minority of compounds $\left(\sim 10^{1}\right.$ out of $\left.\sim 10^{5-6}\right)$ are selected that will pass on to the next stage of development. HTS technology has following characteristics:

- Extremely large numbers of compounds (few thousand to several hundred thousand) are tested [Hertzberg 2000].

- To process such a high volume of compounds, specially designed rapid testing methods are used. Presently, automated robots, high density well plates (normally a plastic plate with a number of small wells, typically 96,384 and up to 1536 wells per plate) and sophisticated software are being used to achieve this goal [Huser 2007]. HTS throughput rates have steadily increased from 10,000 compounds per week rate in late 1980 s to $10,000-100,000$ compounds per day during late 1990s Major 1998 Sundberg 2000].

- The process is cost and capital intensive (large consumption of compounds, sophisticated instrumentation and software).

- The method is "brute force" in nature because it is largely indiscriminate with respect to selecting the compounds to be tested.

There has been a shift towards the use of larger and larger compound libraries (10000 to 1000,000 [Major 1998 Sundberg 2000]. More sophisticated systems to screen larger libraries are being developed and used [Hughes 2001]. Using higher density well plates is one approach to the screening of larger libraries [Hertzberg 2000]. Higher 
Master's Thesis - Sarvesh Upadhyaya - McMaster University - Mechanical Engineering

density wellplates gives the advantages of higher throughput rates and smaller reagent consumption per compound.

These reasons have pushed the HTS industry to undergo an evolutionary process, starting with 96 wells/plate and going towards 1536 wells per plate [Sundberg 2000]. Several researchers have demonstrated higher well plate densities as well. For example, Mere et. al. [Mere 1999] has used a 3456 well format. However, with higher density plates, due to technology limitations, a number of problems can occur [Sundberg 2000]. The major problems are: fast evaporation of liquids due to larger surface to volume ratios at smaller dimensions, problems in liquid handling, and higher costs due to high end precision instrumentation requirements. Hence, practically the well densities are limited to 3456 well/per plate. These problems have motivated researchers to look for alternative technologies.

\subsection{CELL BASED HTS}

This is a type of HTS in which the testing method is based on viable biological cells. It has the following characteristics

- When compared with other assays, e.g. enzyme assay, the data generated are a better representation of an actual human body response. The leads generated are of better quality and have a lower chance of failure in subsequent stages [Khandurina 2002]. 
Master's Thesis - Sarvesh Upadhyaya - McMaster University - Mechanical Engineering

- Sometimes additional factors (e.g. molecules that can alter the behavior of target protein) are present in cells that significantly alter the pharmacology. Such factors might be absent in other types of assays. [Christopoulos 1999, Croston 2002].

- Biological cells are very complicated in their structure and function, and different parts of the cell can elicit different responses to a drug.

- Multi-parameter measurements, called High Content Screening (HCS), can be done [Liptrot 2002]. HCS yields data with enriched bioinformation content and can be done only with cell based HTS [Giuliano 1997].

- It is generally costlier when compared with other assays [Khandurina 2002].

In spite of higher costs, due to its unique advantages, this method is becoming increasingly popular [Khandurina 2002].

\subsection{MICROFLUIDICS FOR CELL BASED HTS}

Microfluidics has the unique capability to work with biological cells at higher throughput levels. For example:

- Very small wells, hence large number of wells on single plate can be made. This would result in ultra-high throughputs currently not possible with well plate technology. 
Master's Thesis - Sarvesh Upadhyaya - McMaster University - Mechanical Engineering

- Ultra small volume liquids (pico to nano liter) can be easily handled. Typically microfluidic devices are closed in nature hence evaporation of liquids is not a problem.

- Cell-cell and cell-extra-cellular matrix interaction are very important parameters in cell behavior [Huang 1999, Wang 1993]. With the help of microfluidics these interactions can be simulated and controlled. For example some molecules can be supplied only to part of a cell [Takayama 2001], mechanical forces can be exerted [Leclerc 2006] and effects can be studied [Chen 1997].

Due to these reasons, the field of microfluidics cell based HTS has significant potential and a device based on this technology would be an excellent addition to current HTS technology [Pihl 2000]. A number of research efforts aimed towards this goal have been published. Detailed discussions about them would be done in next chapter. 


\section{CHAPTER 3}

\section{MICROFLUIDIC DEVICES FOR CELL BASED}

\section{HTS}

Over the last several years, development of cell-based HTS technology capable of providing valuable information on potential drug targets has been a major research area in microfluidics [Khandurina 2002]. Biological cells have been used in a number of ways on microfluidic platforms. Microfluidics has ability to work at small volumes and lengths; hence as compared to their conventional counterparts these devices have been able to achieve higher throughput. For example, Sinclair et. al. developed an HTS ionchannel reader by combining traditional pipette based ion-channel studies with microfluidics [Sinclair 2002, Sinclair 2003]. In this device a single cell attached to a probe is used to scan compounds in a very short time ( $\sim 9$ seconds) at the exit of 32 parallel microchannels. As compared to conventional methods this approach increased the throughput by 2 orders of magnitude. By using microfluidic gradient generator, a device capable of performing cell chemotaxis (migration of cells in response to presence, absence or gradient of chemicals) with precise spatial and temporal controls, has been developed [Jeon 2002]. Detailed accounts of use of microfluidics in cell based HTS have been well compiled by other researchers [Sundburg 2000, Pihl 2005, Pihl 2005b]. A few of the more prominent devices are discussed in following sections. 
Master's Thesis - Sarvesh Upadhyaya - McMaster University - Mechanical Engineering

\subsection{PREVIOUS WORKS FOR CELL CULTURE BASED HTS}

Hung et. al. [Hung 2004] fabricated a device capable of culturing cells in a microarray (array of microsize features) format for long durations. Microfabrication technology was used in fabricating a number of tiny wells (1 $1 \mathrm{~mm}$ diameter) in an array format and connecting microchannels. The schematic of their device is shown in Figure 3.1 .

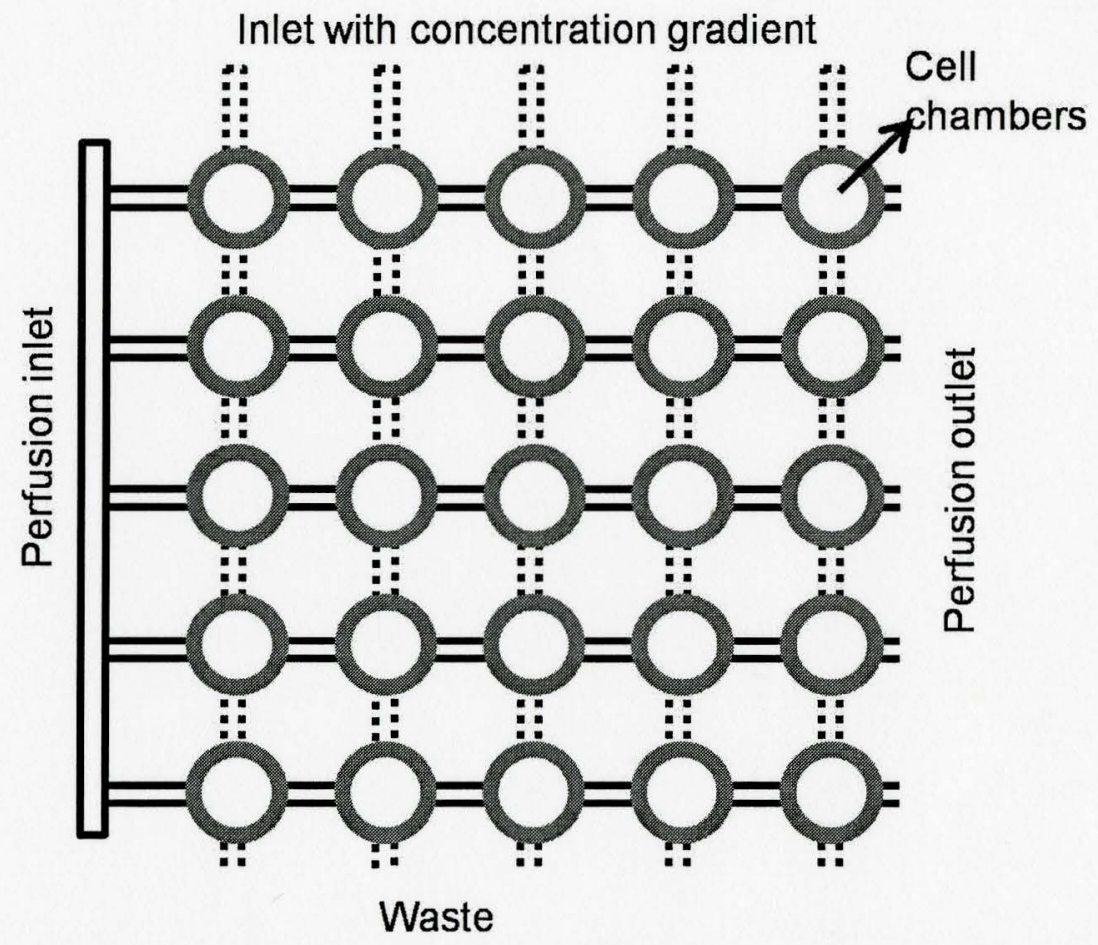

Figure 3.1 Schematic of continuous perfusion device for cell culture: Using microchannels, the device could generate a gradient of concentrations. Cells were cultured in individual wells and microchannels were used for nutrient supply, cell loading and waste collection [Hung 2004].

The device is made of Polydimethylsiloxane (PDMS) and have $10 \times 10$ array of chambers each connected to a network of microchannels. Cells were cultured in 
Master's Thesis - Sarvesh Upadhyaya - McMaster University - Mechanical Engineering

individual wells and microchannels were used for nutrient supply, cell loading and waste collection. The device was run for 16 days culturing HeLa cells. Typically $20-40$ cells could be grown in each chamber. The device also has the capability of gradient generation; hence it can easily test effects of a single compound at various concentrations. This device can be useful in HTS, quantitative cell biology, and bioinformatics.

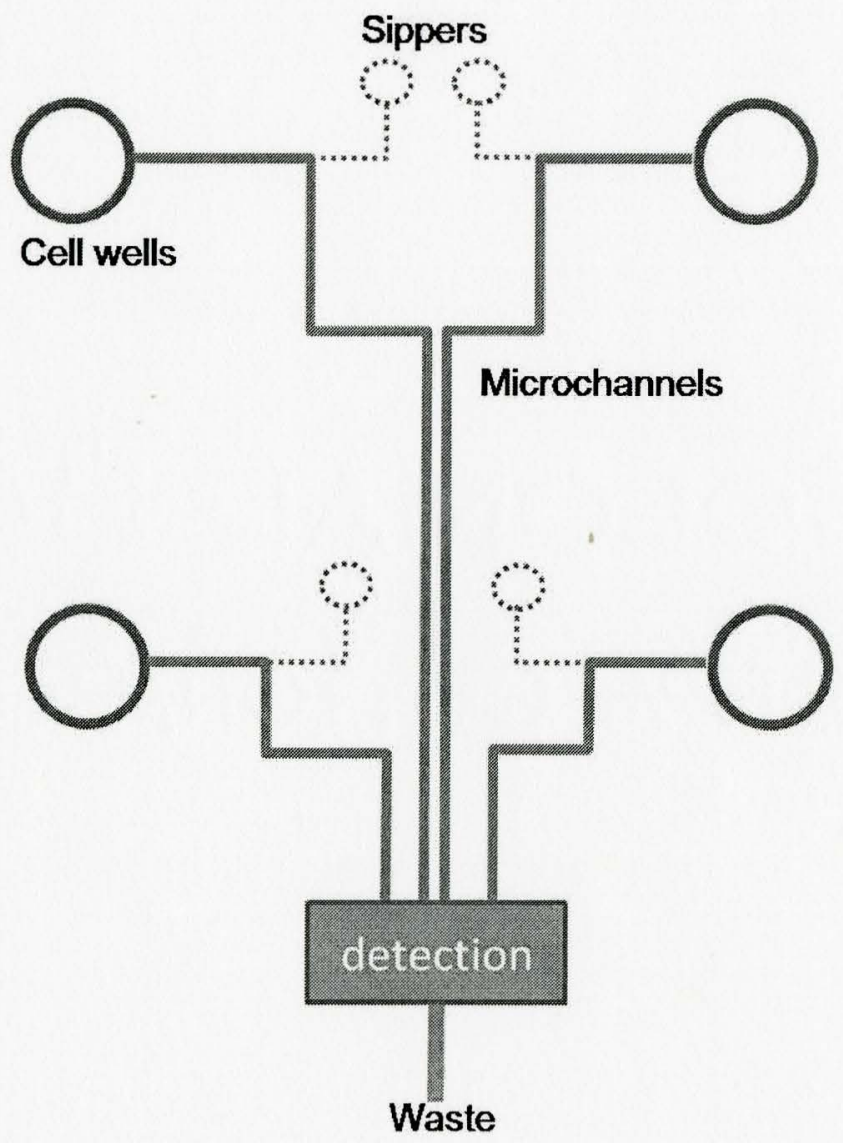

Figure 3.2 Schematic of Caliper Life Sciences microfluidic HTS device: Fabricated on a glass plate, the device can work with various types of HTS including cell based HTS. A number of such platforms could be integrated on a single chip to get higher throughputs [Pihl 2005, Tran 2005]. 
Master's Thesis - Sarvesh Upadhyaya - McMaster University - Mechanical Engineering

Caliper Life Sciences USA has developed a microfluidic cell based HTS device [Pihl 2005, Tran 2005]. The device, as shown in Figure 3.2, has a number of chambers (e.g. 8,12$)$ on a chip. The contents of these chambers (e.g. cells) are sucked (sipped) by microchannels. After being sipped the samples are mixed with other compounds (sipped from wellplates) necessary for analysis. The mixing happens in microchannels which also serve as incubation chambers. After this the analysis mixtures are taken to a detection zone and analysis is carried out serially. Using this device compound, enzyme and substrate consumptions are reported to be $1 / 500,1 / 100$ and $1 / 40,000$ times lower than those of well plates [Pihl 2005]. Presently, this system is used by pharmaceutical companies for cell based HTS and commercially available from Caliper Life Sciences USA.

Sabatini et.al. [Ziauddin 2000] have reported creation of array of micro-spots of cDNA (complementary DNA) mixed gelatin on a glass slide. They were able to make 140 to 192 such spots on a $40 \times 20 \mathrm{~mm}$ glass slide. Cells were subsequently cultured on the gelatin. cDNAs in individual spots were different hence the cells growing on top of each spot would express different proteins. As compared to 1536 wellplate wells $(\sim 2 \mathrm{~mm}$ diameter), the achieved spot sizes were much smaller $(120-140 \mu \mathrm{m})$. The technology has the capability to create $6,000-10,000$ spots on a single glass slide. Device schematic is shown in Figure 3.3. This format maintains uniform physiologic environment and does not restrict cell growth by compartmentalization of culture area. However, dosage of the cDNAs is passive and dependent on slow release from the gelatin spot. Morover to create the cDNA spots, robotic arms were used which would limit the potential advantages 
Master's Thesis - Sarvesh Upadhyaya - McMaster University - Mechanical Engineering

microfluidics technology can provide. Robotic arms also limit the throughput as its functioning is serial in nature.

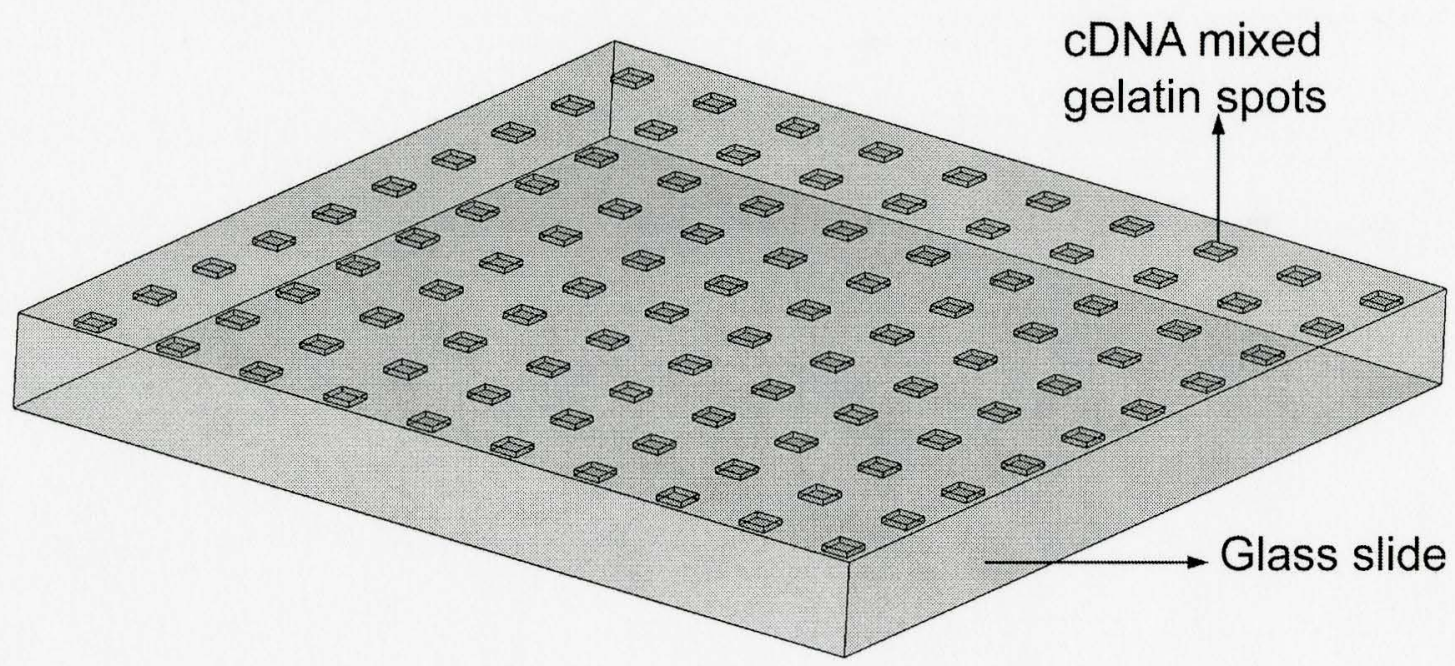

Figure 3.3 Schematic of cDNA array device: Robotic arm was used to create cDNA mixed gelatin spots on a glass slide. Top glass surface was flat without compartments and cells were grown on it [Ziauddin 2000].

\subsection{LIMITATIONS}

The major limitations found in present devices can be grouped as following.

\subsubsection{Downsizing of Macro World Approaches}

Most researchers [Hung 2004, Walker 2002, Powers 2002] have tried to miniaturize existing well plate architecture with the help of microfabrication technology (i.e. miniaturization of wells). But as dimensions decreases the phenomena, that are not very important at macro scale, start to become dominating [Wautelet 2001]. The uniqueness of microfluidics cannot be fully exploited by following the traditional macroworld approaches. 
Master's Thesis - Sarvesh Upadhyaya - McMaster University - Mechanical Engineering

For example, Ziauddin et al. [Ziauddin 2000] used state of art robotic arms to create $120-140 \mu \mathrm{m}$ spots, which is an attempt to make smaller spots using macroscale approach. Similarly, the key difference between the device developed by Hung et. al. [Hung 2004] and well plates are that the size of wells is smaller $(\sim 1 \mathrm{~mm}$, as compared to $\sim 2 \mathrm{~mm}$ for 1536 well plate) which results in higher well density. Layout of wells in their device and well plates are identical with the difference of having microchannels for nutrient supplies.

\subsubsection{Use of Non-Traditional Cell Culture Materials}

The fabrication technologies used in microfluidics have been developed in association with microelectronics industry. As a result, materials (e.g. silicon, SU8) used in microfluidics are not always biocompatible. In previously reported devices [Hung 2004, Walker 2002, Powers 2002], non-traditional materials like Si and PDMS were used as cell culture substrates. The cell culture and behavior have been shown to be dependent on culture substrate [Helgason 2005]. For example, as per substrate toxicity studies [Ertel 1994, Kaul 1985, Tampion 1987] there is a significant difference between cell mortality and growth rates when cultured on traditional materials (tissue culture polystyrene -TCPS, glass) as compared to that of non-traditional materials (PDMS, Polyetherurethane). When cells were cultured ( 2 hours duration, bovine aortic endothelial cells-BAEC in protein less media) on PDMS the cell death rates $(93 \% \pm 5 \%)$ are 10 times and 21 times larger than that of glass $(9 \% \pm 3.5 \%)$ and TCPS $(4.5 \% \pm 6 \%)$ respectively. When cells were cultured in presence of protein media the cell death ratios for PDMS to glass and PDMS to TCPS were 35 and 48 respectively [Ertel 1994]. Moreover in contrast 
Master's Thesis - Sarvesh Upadhyaya - McMaster University - Mechanical Engineering

to glass and TCPS, cell viability on PDMS has strong dependence with cell density. For BAEC cells grown for two hours in PDMS, cell mortality changed from $93 \%$ at $10^{3}$ cells $/ \mathrm{cm}^{2}$ to $20 \%$ at $10^{6}$ cells $/ \mathrm{cm}^{2}[$ Ertel 1994]. Similarly cell growth rate on polyetherurethane was 5 times slower than that of glass [Ertel 1994].

The physiological response of cells, the most important parameter in cell based HTS, might change due to use of new substances. Culturing on new substrates would make historical characterization of cell culture non-applicable.

\subsubsection{Compartmentalization of Cell Culture Area}

The researchers have tried to divide the chip into a number of chambers either in form of wells or in form of microchannels [Hung 2004, Walker 2002]. The typical microfluidic dimensions are in the same of order of magnitude as that of typical cells. With decreasing chamber sizes the growth of cells would get hampered. More ever, due to larger surface to volume ratio cells would experience more of cell-to-wall interaction as compared to cell-to-cell interaction. This may lead to changes in the cell behavior as cell functioning is highly dependent on cell-to-cell interactions and confinements [Helgason 2005]. For example, insect cells cultured in microchannels grew significantly slower than those grown in flasks [Walker 2001]. Cells population of cells grown in microchannels made of glass and PDMS grew only by $51 \%$ and $37 \%$ while in same time cell population in flask doubled [Walker 2001]. Similarly, the growth dynamics of embryos in microchannels is much different than what is observed at macroscale cultures [Raty 2001]. 
Master's Thesis - Sarvesh Upadhyaya - McMaster University - Mechanical Engineering

From above discussion it can be concluded that a number of microfluidic cell HTS devices have been developed. But there are a number of limitations in them including compartmentalization and use of non-traditional cell culture materials. To overcome these limitations a new approach is discussed in next chapter. 


\section{CHAPTER 4}

\section{NANOPOROUS MEMBRANE BASED}

\section{MICROFLUIDIC DEVICE FOR CELL BASED}

\section{HTS}

\subsection{THE IDEA}

In contrast to existing devices, a multi-layered non-compartmentalized, cell culture approach has been developed. Figure 4.1 shows the schematic of the approach. The idea is to use agar gel as cell culture substrate, similar to agar plates, and to use nanoporous membrane in conjunction with microfluidic channel to supply drugs in agar gel. From the gel the drugs would reach to cells by diffusion. As shown in Figure 4.1, the drugs are supplied into the gel in a microarray (an array of microsize features) format. This microarray is analogous to wells of a well plate.

\subsection{ADVANTAGES OVER PREVIOUS WORK}

In previously reported works, [Hung 2004, Walker 2002, Powers 2002] both silicon and PDMS were used as cell culture substrates. In this present approach, the cell culture is carried out on an agar gel surface. This offers a number of advantages, for 
Master's Thesis - Sarvesh Upadhyaya - McMaster University - Mechanical Engineering

example, agar is a traditionally used cell culture material [Freshney 1994] and due to historic use its wide characterization as a cell growth medium is available. As there are similarities between the mode of cell culture in this device and that of traditional cell culture, it would also lead to easy integration with existing cell culture protocols.

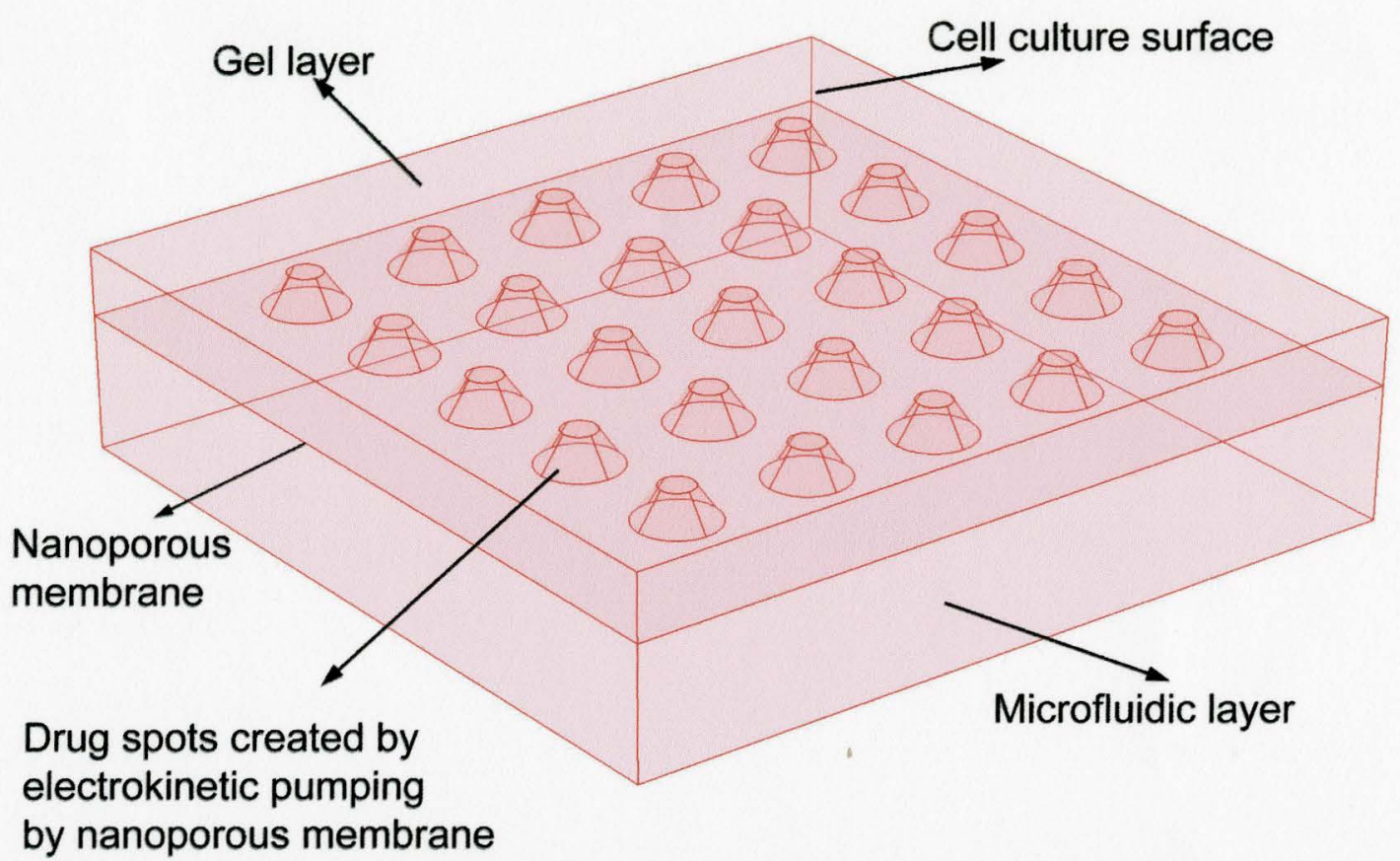

Figure 4.1 Schematic diagram of the approach for cell based HTS: Cultured cells are not compartmentalized while the gel acts as the culture substrate. It is a three layered architecture. The bottom layer consists of microfluidic channels that are filled with drugs solution. Middle layer works as selective pump. Top layer is the gel layer meant for cell culture.

In contrast to previously reported devices [Hung 2004, Powers 2002], there is no compartmentalization for the cell culture area as cells are cultured on a flat gel surface. This offers many advantages. For example, the culture surface area is analogous to cell culture agar plates, i.e. a flat agar gel sheet and cells cultured on top surface. Unlike 
Master's Thesis - Sarvesh Upadhyaya - McMaster University - Mechanical Engineering

previously reported cell culturing in microchannels and microwells [Walker 2001, Raty $2001]$ in this case cell behavior changes due to small well sizes are not expected. As there is no compartmentalization on cell culture area, unlike other microfluidic devices, cell-tocell interactions are not expected to get hampered. Cell-cell interactions are very important in cell behavior, for example: cell proliferation, cell differentiation [Greenwald 1992] programmed cell deaths [Ellis 1991] and change in gene expressions [Streuli 1991].

The drug pumping, which is done by nanometer size pores, can be controlled very precisely by controlling the applied electric potentials. Theoretically, by having a single nanopore to do the pumping, the dosing can be controlled down $10^{7}$ molecules/second limits [Reber 2001, Macpherson 2002].

\subsection{WORKING PRINCIPLE}

The complete functioning of the device is dependent on a number of factors, namely: pumping of drug molecules through a nanoporous membrane, drug spot formation, diffusion of drugs in gel and cell culture. Each of these factors has to be carefully evaluated and the device designed appropriately. The design of these sub-units is discussed in more detail in the subsequent sections.

\subsubsection{Nanoporous Membranes and Pumping}

Nanoporous membranes, in the current context, are thin $(\sim 10 \mu \mathrm{m})$ flexible membranes having narrow $(10-100 \mathrm{~nm})$ through holes. A variety of membranes is 
Master's Thesis - Sarvesh Upadhyaya - McMaster University - Mechanical Engineering

commercially available and differs in the size of the pores (the holes) and the densities (number of pores per unit area). The membrane can be hydrophilic or hydrophobic depending upon its material (membrane details in section 5.1.3). When a hydrophilic membrane is immersed in aqueous solutions the pores will be filled due to capillary forces.

Upon application of an electric field through the membrane, the solution-filled nanopores start to act like a micropump [Kuo 2003]. The actual pumping mechanism is complex and employs principles of electroosmosis, electrophoresis and diffusion [Kemery 1998]. The pumping magnitude and direction depends upon a number of parameters (e.g. electric field, solute ions, concentrations, solution $\mathrm{pH}$, pore size, pore density, molecular size and molecular charge).

\subsubsection{Electroosmotic pumping}

Figure 4.2 shows the nanoporous membrane electroosmotic pumping mechanism. Electroosmotic [Rice 1965, Chen 2002] pumping occurs when an electrical field is applied through a channel having electrically charged walls. Electrically charges walls occur due to ionic exchange between wall and solution molecules. For example, when water comes in contact with PDMS, PDMS surface acquires a negative charge due to loss of protons and formation of $\mathrm{SiO}^{-}$groups at the surface. The charged wall surface repels similarly charged ions from the solution near the walls and attracts oppositely charged ions from the bulk solution. The net excess of ions near the walls form an electrical 
Master's Thesis - Sarvesh Upadhyaya - McMaster University - Mechanical Engineering

charged layer called the double layer. The double layer is made up of two distinct layers: the Stern layer and the diffuse layer. The Stern layer consists of a single ionic layer. It is firmly attached to the walls due to very strong electrical fields (E $10^{7}$ to $10^{8} \mathrm{~V} / \mathrm{m}$ ) present between the Stern layer and the wall. The diffuse layer is loosely attached to the walls.

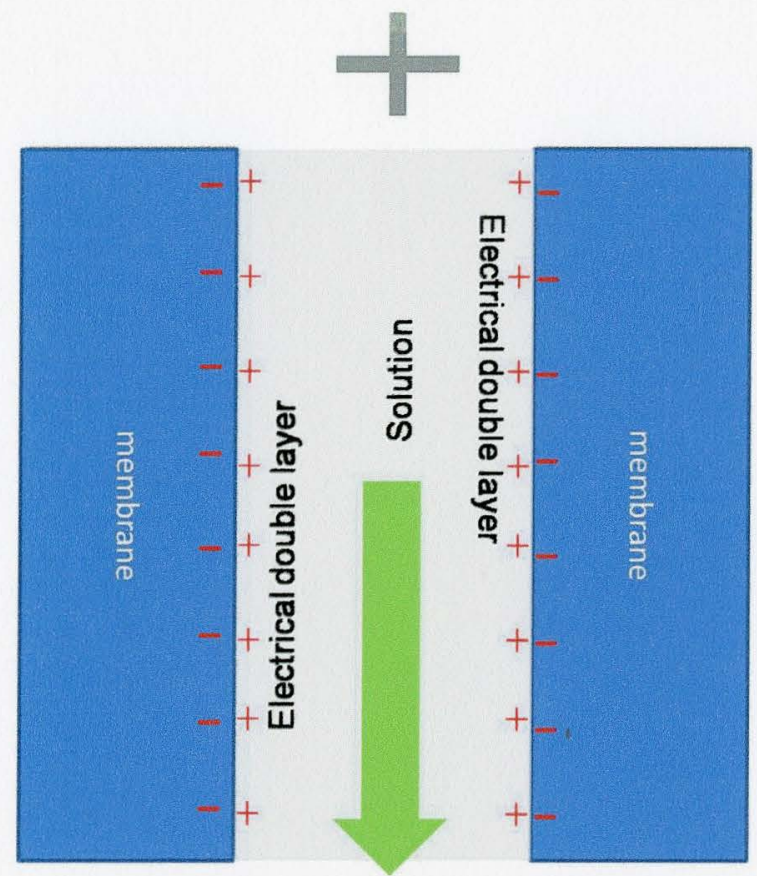

Fluid Flow Direction

Figure 4.2 Electroosmotic pumping: Due to interactions between the molecules on the membrane wall and solution, a charge develops at the membrane surface. This charge causes an electrical double layer to develop in the solution. Upon application of an electric field, the solution moves with the motion of double layer.

Upon application of an electric field, the diffused layer moves in the direction of electrostatic force i.e. towards positive electrode if zeta potential is negative or vice versa. 
Master's Thesis - Sarvesh Upadhyaya - McMaster University - Mechanical Engineering

Due to viscosity, the motion of diffused layer exerts a force on solution present in channel resulting into motion of the solution. This motion generates a shear plane between the Stern layer and the double layer. The electrical potential at this shear plane is called the zeta potential. The zeta potential (typically 1-200 mV) is used as a measure of electroosmotic pumping tendency at any surface-solution interface [Kirby 2004]. The pumping magnitude and direction both depend on the zeta potential and the applied electric field. Volume flow rate due to electroosmotic (in absence of pressure gradient) in a capillary can be given by following equation [Rice 1965]:

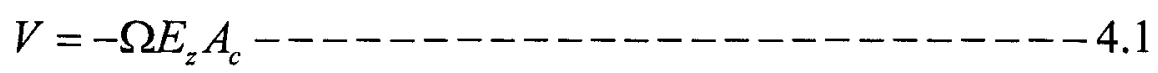

Where $E_{z}$ is applied electric field, $A_{c}$ is cross sectional area of the capillary, and $\Omega$ is given by

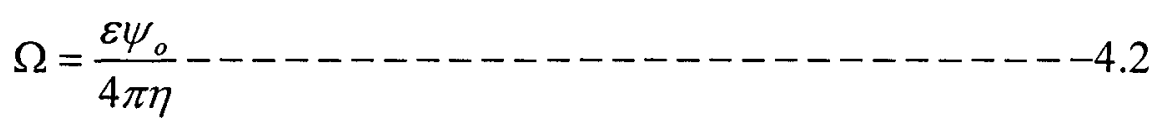

In equation $4.2, \varepsilon$ is dielectric constant of fluid, $\eta$ is coefficient of viscosity, and $\psi 0$ is zeta potential.

Hence electroosmotic flow can be controlled by a number of parameters, like $\varepsilon, \eta$, $\psi_{0}, E_{z}, A_{c}$, these parameters in turn can controlled by membrane pore size, pore material, solution ionic concentration and electric field. 
Master's Thesis - Sarvesh Upadhyaya - McMaster University - Mechanical Engineering

When an electric field is applied across the membrane, the electric field lines pass through the nanopores because the membrane is made of an insulating material. The pores, upon immersion in solution, develop a zeta potential and start to pump electroosmotically.

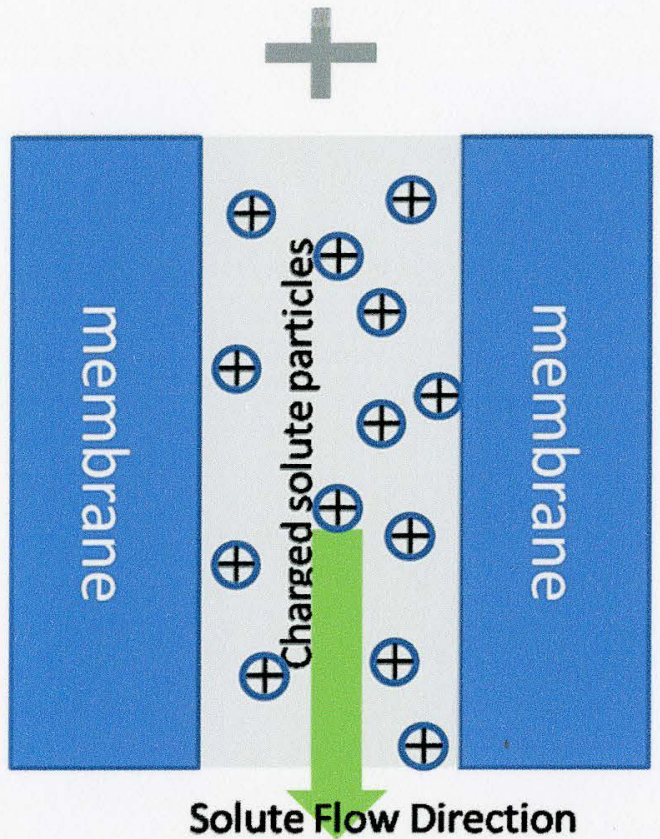

Figure 4.3 Electrophoretic pumping: When a charged particle or a molecule is present in an electric field, the particle starts to move. If this potential is present across a membrane, the particles would cross the membrane from one side to other.

\subsubsection{Electrophoretic pumping}

Schematically, the electrophoretic pumping mechanism is shown in Figure 4.3. Electrophoretic pumping occurs when an electrical field is applied in a channel filled with a solution containing charged particles or molecules [Manz 1994]. 
Master's Thesis - Sarvesh Upadhyaya - McMaster University - Mechanical Engineering

When a charged species is present in an electric field the developed electric force can be described by equation 4.3. [Manz 1994].

$$
\vec{F}_{e}=z_{p} e \vec{E}-----------------(4.3)
$$

Where: $F_{e}{ }^{\text {is }}$ the electric force, $z_{i} \times e$ is the net charge on the particle and $E$ is the electric field.

Due to this electrostatic force, the particle starts to move. This motion generates a drag force given by equation 4.4 .

$$
\overrightarrow{F_{f}}=-f_{p} \vec{V}_{p}-----------------(4.4)
$$

Where $F_{f}$ is frictional force, $f_{p}$ is the friction coefficient of the particle and $V_{p}$ is velocity of the particle. In equation 4.4, in contrast to macroscopic flows where drag force is proportional to square of velocity, the drag force is linearly proportional to velocity. This is due to the fact that in electrophoretic flows the velocities are very small, hence Reynolds number is very small too. This means the viscous drag force (varies linearly with velocity) dominates over inertial drag force [OBrien 1978].

At equilibrium, the electrical and frictional forces are balanced. From equation 4.3 , and 4.4 , the following equation gives the relationship between the velocity of a particle and the applied electric field. 
Master's Thesis - Sarvesh Upadhyaya - McMaster University - Mechanical Engineering

$$
\vec{V}_{p}=\left(\frac{z_{p} e}{f_{p}}\right) \vec{E}-----------------(4.5)
$$

The summation of motions of a large number of particles (molecules) yields electrophoretic pumping. An electric field across the nanoporous membrane causes the solute particles to move through the nanopores from one side of the membrane to other side. The magnitude of electrophoresis can be controlled by controlling the charge on the particle, the applied electric field, the frictional properties of solution, the pore size and density. For example increasing the pore density will proportionally increase the pumping magnitude (assuming pumping of one pore does not affect other pores). Higher molecular charges would increase the pumping and a charge reversal will change the pumping direction. This charge reversal can be important in case of proteins since their molecular charges can switch from positive to negative or vice versa depending upon solution $\mathrm{pH}$ [Xia 2007]. This in turn will change the protein pumping direction depending upon $\mathrm{pH}$.

\subsubsection{Diffusional mass transport}

By assuming pores to be perfect cylinders of length equal to the thickness of membrane, the diffusional flux of uncharged molecule occurring through a single pore can be given by following equation [Bean, 1972, Rostovtseva 1996]:

$$
\phi=D\left(\frac{\pi r^{2} \Delta c}{(t+\pi r / 2)}\right)-------------(4.6)
$$


Master's Thesis - Sarvesh Upadhyaya - McMaster University - Mechanical Engineering

Where $\mathrm{D}$ is diffusion coefficient, $\mathrm{r}$ is radius of the pore, $\Delta \mathrm{c}$ is concentration difference across the membrane, and $t$ membrane thickness. In above equation hindrance provided to diffusion due to very small pore sizes are neglected. This hindrance can be accounted for by correcting diffusion constant as given by following equation [Bohrer 1984]:

$$
D_{e}=D\left(\beta \frac{f_{\infty}}{f_{\lambda}}\right)
$$

Where $D_{e}$ is effective diffusion coefficient. $f_{\infty}$ and $f_{\lambda}$ are molecular friction coefficients in free solution and pore respectively. $\beta$ and $\lambda$ are calculated from pore radius $r$ and molecular radius $r_{m}$ by following equation:

$$
\lambda=\frac{r_{m}}{r} \text { and } \beta=(1-\lambda)^{2}
$$

\subsubsection{Relative magnitude of various pumping modes}

Out of three modes of mass transfer (diffusion, electroosmotic and electrophoretic), for typical operating conditions used in this study $(10-100 \mathrm{~nm}$ pores, open pore patch area of 0.04 to $0.25 \mathrm{~mm}^{2}, 8 \mu \mathrm{m}$ membrane thickness) the relative magnitude of diffusional transport is much smaller as compared to electroosmotic or electrophoretic [Kuo 2003]. The microchannel layout of the device is made so as to avoid any pumping pressure development due to electroosmotic or electrophoretic flows occurring in microchannels. This is achieved by opening the one end of microchannels to 
Master's Thesis - Sarvesh Upadhyaya - McMaster University - Mechanical Engineering

atmosphere, hence any pressure development in microchannels will lead to flow towards atmospheric outlet (because flow through microchannels has less resistance as compared to flow through nanoporous membrane which has high flow resistance due to nanometric pore sizes). In the absence of pressure buildup from microchannels, for charged molecules the pumping is expected to be dominated by electrophoretic flow [Kuo 2003]. For neutral molecules electroosmosis will dominate.

\subsubsection{Drug Spot Formation}

As discussed, when an electric field is applied through a nanoporous membrane, the pores start to behave as tiny pumps. All the pores, except those in selected regions, are blocked by filling the pores with liquid Polydimethylsiloxane (PDMS) and solidifying the PDMS inside the pores by application of heat (details in section 5.2.4). Blocking is done as to leave an array of small patches having a number of open pores. Each such patch on a membrane, consisting of $1000-3000$ pores, is used as a single micropump. These micropumps act as the interface between the gel and the microchannel. As shown in Figure 4.4, in the presence of an electric field, the drug flows from the microchannel into the gel layer (or vice versa depending on the charge on the molecule and electric field direction). Use of vertical through hole is essential for creation of well defined drug spots. In such membrane drug can travel only in thickness direction without moving in latter direction as pores does touch each other. Interconnected pores would lead to lateral motion of molecules in the membrane itself and would lead to mixing of drugs from 
Master's Thesis - Sarvesh Upadhyaya - McMaster University - Mechanical Engineering

adjacent spots. Hence polycarbonate membrane having vertical non-connecting through nanopores (section 5.1.3) will be used for drug spot formation.

Initially the drug is pumped into the bottom most region of the gel into an area precisely matching the open pore patch. After pumping, due to electrophoretic mobility of drug molecules in the gel slab, the drug starts migrating in the gel [Wieme 1965]. This migration, depending upon the electric field distribution, allows the drug to be distributed in a $3 \mathrm{D}$ region in the gel. This $3 \mathrm{D}$ region is referred to as a drug spot.

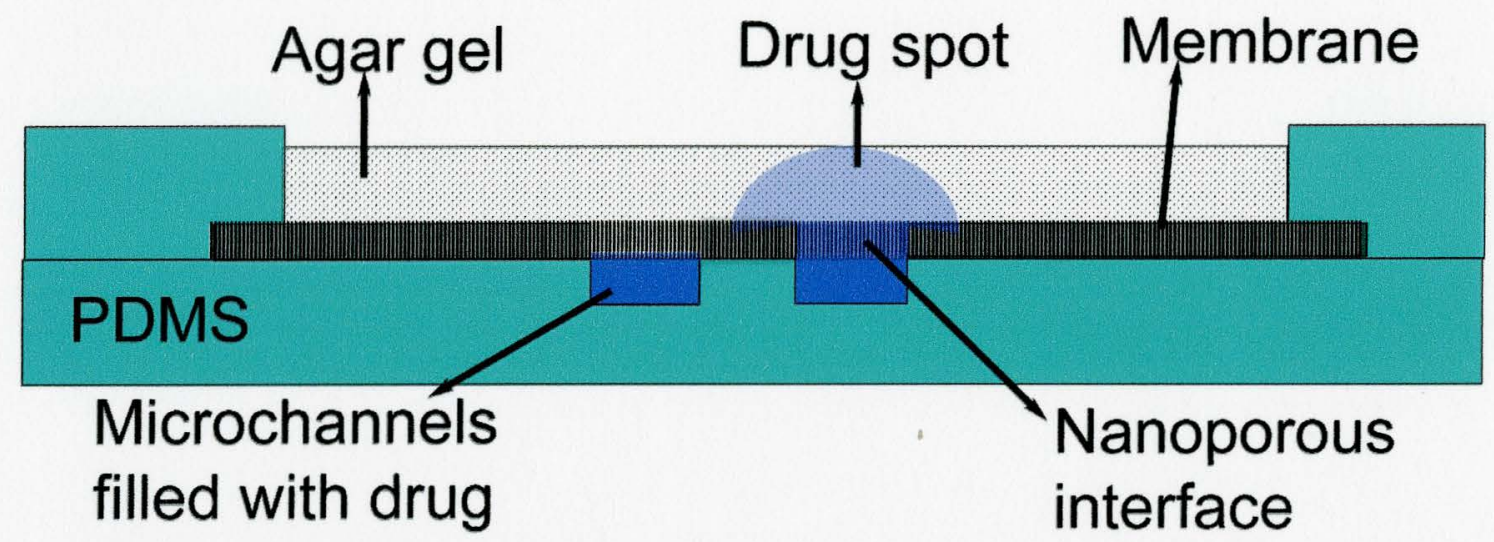

Figure 4.4 Schematic of device working: A nanoporous membrane is sandwiched between the bottom layer containing microchannels and the top layer of gel. Upon application of an electric field, the drug molecules move into precisely defined microspots in the gel.

There are a number of open pore patches arranged in an array format on the membrane. The microchannel is aligned with these patches. Each patch behaves as a single micropump and forms a single drug spot in the gel layer. By having a number of such patches a microarray of drug spots is formed in the gel. The individual spots in an 
Master's Thesis - Sarvesh Upadhyaya - McMaster University - Mechanical Engineering

array can have the same drug or different drugs depending upon the contents in the microchannels below them.

\subsubsection{Molecular Diffusion in Gel}

Initially, the drug spots are created in the bottom region of the gel (i.e. the gel region near the membrane). The agar gel contains a large percentage ( $99 \%)$ of water and is very porous in nature (details about agar in section 5.1.4). This allows the diffusion of molecules within the agar gel. A drug concentration gradient is established between the drug spot and the bulk gel. Hence, diffusion of drug molecules occurs causing the initial size of the drug spot to grow. This diffusion can be divided into horizontal and vertical components. As shown in Figure 4.5, the vertical diffusion causes the drug to diffuse towards the top surface of the gel increasing the thickness of the spot. Vertical diffusion ultimately leads the drug to reach the top surface of the gel where cells will be cultured. The horizontal diffusion causes the spot size to grow laterally increasing the drug spot diameter. With the drug spot diameter increase, the gaps between adjacent spots decrease. A large horizontal diffusion can potentially lead to undesirable mixing/interaction of adjacent spots.

According to the preceding analysis, it can be concluded that horizontal diffusion is undesirable, while vertical diffusion is essential for device functioning. The gel has isotropic properties, hence both horizontal and vertical diffusion constants have identical values (e.g. $3 \times 10^{-11} \mathrm{~m}^{2} / \mathrm{s}$ for $2 \%$ agar gel at room temperature [Ackers 1962]). However, 
Master's Thesis - Sarvesh Upadhyaya - McMaster University - Mechanical Engineering

by optimizing the geometry of the gel (e.g. a thinner gel with more precisely positioned spots) the net effect of diffusion can be optimized.

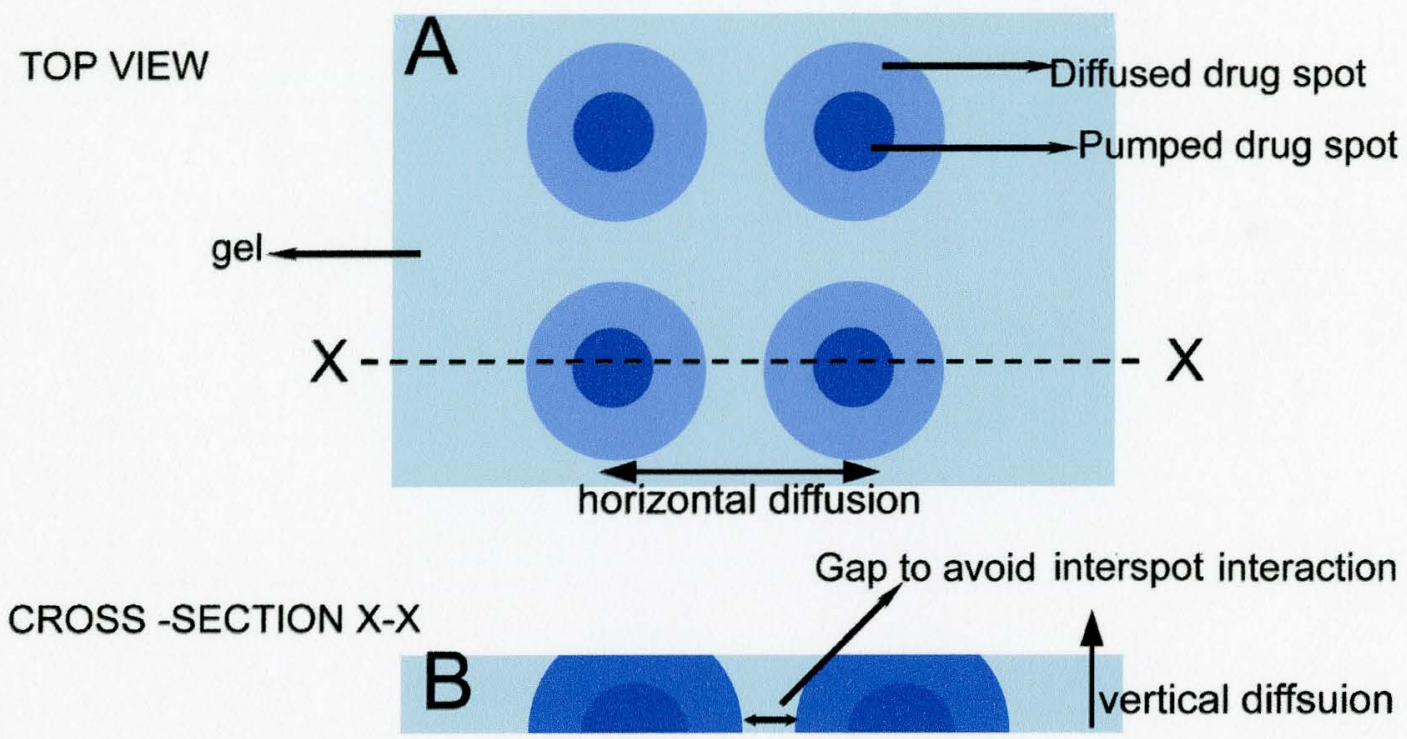

Figure 4.5 Schematic of drug diffusion in agar gels: Darker regions represent drug actively pumped by electrophoresis. Lighter blue regions define regions of drug migration due to molecular diffusion. (A) Horizontal diffusion causes the spot diameter to grow. (B) Vertical diffusion causes spot thickness go grow. It is essential for device to function properly.

\subsubsection{Cell Culture}

The cells will be cultured on the top surface of the gel layer analogous to conventional cell culture on agar plates, hence no specialized procedure is required for culturing of the cells. The agar gel cell culture has been well characterized [Madigan 2005]. Analogous to agar plates, cells in the device will remain in a continuous lawn configuration. As compared to other microfluidic device cell culture [Hung 2004, 
Master's Thesis - Sarvesh Upadhyaya - McMaster University - Mechanical Engineering

Walker 2002, Powers 2002], there are a number of advantages of this noncompartmentalized agar cell culture and are given in sections 3.2.2 and 3.2.3. The drug microarray, as explained above, lies just below the culture lawn. Cells present directly over a particular spot experience the concentration of drug present in that spot. Because the spots do not touch one another, a large number of parallel experiments can be performed on a single, continuous cell culture lawn.

\subsection{DEVICE DESIGN}

\subsubsection{Design Criteria and Parameter Selection}

The following design parameters were developed for the thesis work:

4.4.1.1 Device structural material: Polydimethylsiloxane PDMS based soft-lithography [Xia 1998] has recently been well characterized, and widely used. [Whitesides 2002]. PDMS is transparent and rubbery. These properties will be useful for easy visualization and leak proof assembly. This method does not require a clean room environment and the time to prototype is quick.

Base on above reasons PDMS is used as structural material of the devices.

4.4.1.2 Pore size: The device is designed for a number of different molecules having varied electrical charges and molecular weights. At smaller pore sizes $(<10 \mathrm{~nm})$, the pumping action of the pores becomes molecule dependent, i.e. depending upon molecule charge and size it may or may not get pumped [Kemery 1998]. At a larger pore sizes 
Master's Thesis - Sarvesh Upadhyaya - McMaster University - Mechanical Engineering

$(>1000 \mu \mathrm{m})$, diffusion of drug molecules from the microchannel to the gel can happen without application of any potential. This would result in loss of control of drug dosing precision. .

Based on the above discussion, a starting pore size of $100 \mathrm{~nm}$ diameter was selected for use.

4.4.1.3 Pore density: The pore density has little effect (other than the magnitude of pumping). A larger number of pores per unit area (higher density) give higher drug pumping rates. Commercially available pore density (for $100 \mathrm{~nm}$ pores, $4 \times 10^{8}$ per $\mathrm{cm}^{2}$ ) was used.

4.4.1.4 Membrane material: The membrane should be transparent for easy visualization. Membrane material should be insulating in nature so that electric field lines pass only through pores. It should be economical and easily available. Based on these criteria, polycarbonate was selected as the membrane material.

4.4.1.5 Array dimensions: Array dimensions (array size, interspot distance, individual spot sizes) are important parameters for the proper working and performance of the device. As no previous data is available, a number of devices with different array dimensions were used.

4.4.1.6 Gel material and dimensions: The first requirement is that the gel material should be one that is traditionally used for cell culture. The second requirement is that the gel 
Master's Thesis - Sarvesh Upadhyaya - McMaster University - Mechanical Engineering

should be permeable for the drugs. Based on these criteria, agar was selected as the gel material. Other gel materials with very similar properties (diffusivity, water content, etc.) like agarose are also expected to work similarly.

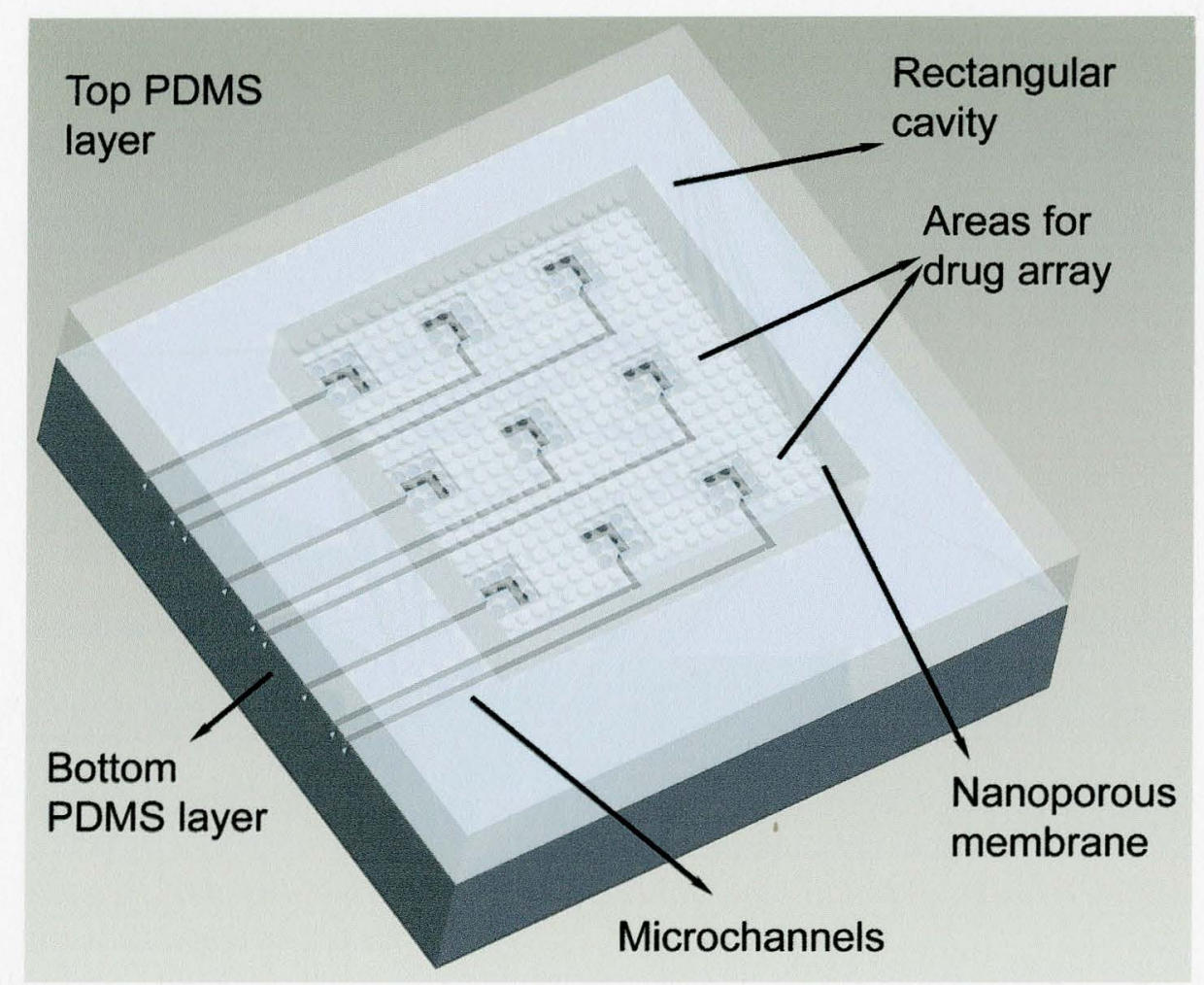

Figure 4.6 3D-schematic of device design. PDMS serves as the supporting structural material for the gel, membrane and channels. Different variations of devices have different patterning of membrane and microfluidic channel.

The dimension of the gel layer affects the diffusion of drugs, so a number of gel dimensions (thickness $0.5 \mathrm{~mm}$ to $3 \mathrm{~mm}$, size $5 \mathrm{~mm} \times 5 \mathrm{~mm}$ to $25 \mathrm{~mm} \times 25 \mathrm{~mm}$ ) were selected for investigation. 
Master's Thesis - Sarvesh Upadhyaya - McMaster University - Mechanical Engineering

\subsubsection{Design Variations}

Based on the above working principle and the basic design (Figure 4.6), a number of devices were designed. These devices are used for different objectives and testing requirements.

The first type of devices is single spot forming devices. These devices form a single spot of drug molecule in the agar gel. These devices are used for the device working demonstration and characterization.

The second type of devices is multiple spot and microarray forming devices. These devices form multiple spots ( 3 spots to 144 spots) of drug in the agar gel and are used to demonstrate the ability of independent control over individual drug spots as well as the ability to form microarrays.

The third type of devices is diffusion barrier device. These devices have a specially designed agar gel layer for optimizing drug diffusion (lower horizontal diffusional effects, higher vertical diffusional effects) in order to maximize drug spot density.

The idea of using nanoporous membrane based electrokinetic pumping to create micro array of drug spots in agar gel for cell based HTS is used to create a number of microfluidic devices. Individual devices are discussed in detail in their respective chapters. 


\section{CHAPTER 5}

\section{FABRICATION AND EXPERIMENTAL}

\section{SETUP}

\subsection{FABRICATION MATERIALS}

Fabrication of devices is a multiple-step sequence of photolithographic and chemical processing involving a number of materials. The following section describes the materials and the common fabrication methods that were used during the preparation of the devices discussed in the latter chapters.

\subsubsection{SU8 Photoresist:}

SU8 is an epoxy based UV light $(365 \mathrm{~nm})$ negative photoresist. Its primary advantage, unlike other low viscosity photoresists, is its ability to make very high aspect ratio microstructures [Shaw 1997]. Using SU8, up to $1200 \mu \mathrm{m}$ thick structures having an aspect ratio of 18 have been fabricated. Before exposure to UV light, the SU8 photoresist is in a viscous liquid ( 2500 cst. centi Stokes) form having $72-85 \%$ solid content dissolved in an organic solvent [Lee 1995]. The SU8 viscosity (hence the film thickness that can be produced) varies depending upon percentage of solid content present in it [Feng 2003]. SU8 polymerization is done in two steps. First, during the UV exposure a strong acid group is generated. In the second step, the photoresist is heated at a 
Master's Thesis-Sarvesh Upadhyaya-McMaster University-Mechanical Engineering

predetermined temperature $\left(\sim 90^{\circ} \mathrm{C}\right)$. During this acid-initiated process, thermally driven epoxy cross-linking takes place [Shaw 1997]. After this polymerization reaction, the typical Young's modulus of SU8 is 4.02 GP (Giga Pascal). [Lorenz 1997]. SU8 having different viscosities are available from MicroChem Corp USA. SU8-100 capable of forming structures varying from 100 to $250 \mu \mathrm{m}$ was used [MicroChem Corp. SU8-100 technical data sheet, Appendix 6].

\subsubsection{Polydimethylsiloxane}

Polydimethylsiloxane, popularly known as PDMS has become widely popular now by the classical work of Whitesides [Xia 1998, Whitesides 2002] at Harvard. PDMS has excellent optical properties for current application (transparent) and it is biocompatible [Whitesides 2001]. PDMS has been extensively used in microfluidics, some of the well known applications include microcontact printing [Kumar 1994], microfluidic network [Delamarche 1997], capillary electrophoresis [Effenhauser 1997], multilayered structures [Unger 2000] and biomedical devices [Fujji 2002].

PDMS was purchased from Dow Corning Incorporation, USA, sold by the brand name of Silgard 84 in uncured form i.e. before polymerization. It was supplied as two separate components (base and curing agent). Polymerization happens on application of heat $\left(60{ }^{\circ} \mathrm{C}\right)$ when a base (short chains containing venyl groups) is cross linked with a curing agent containing hydroxiloxane group [Fu 2003]. After curing it forms a rubbery transparent solid having a molecular structure as shown in Figure 5.1. 
Master's Thesis-Sarvesh Upadhyaya-McMaster University-Mechanical Engineering

The base to curing agent ratio used for making the devices was maintained as 10:1, as recommended by the supplier. For microcontact printing purposes, a thinner mixture is obtained by adding higher percentage (1:3 base to curing agent) of curing agent.

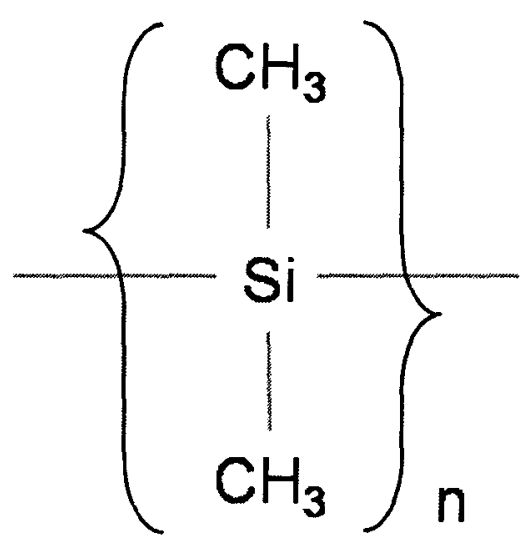

Figure 5.1 PDMS molecular structure.

\subsubsection{Polycarbonate membrane}

It's a thin $(8 \mu \mathrm{m})$ transparent film having through straight nanoporous holes in it. Membranes having different pore sizes $(10 \mathrm{~nm}, 100 \mathrm{~nm}, 1 \mu \mathrm{m})$ are purchased from GE Osmonics. The membrane supplied with polyvinylpyrrolidone coating is wetable. When dipped in water, the water molecules fill the nanopores due to the large capillary action forces that are experienced at smaller scales. As provided by the manufacturer, the pore size distribution falls between $+0 \%$ to $-20 \%$ of the nominal size [GE Osmonics Product Catalog, Appendix 7]. The pore sizes and densities are given in table 4.1.

Electron microscopic picture of the membrane is shown in Figure 5.2 
Master's Thesis-Sarvesh Upadhyaya-McMaster University-Mechanical Engineering

TABLE 4.1 Membrane pore size and density (From manufacture datasheet, Appendix 7)

\begin{tabular}{|l|l|}
\hline $\begin{array}{l}\text { Pore Size } \\
(\text { Tolerance }+0 \%,-20 \%)\end{array}$ & $\begin{array}{l}\text { Pore Density } \\
\text { (Tolerance } \pm 15 \%)\end{array}$ \\
\hline $10 \mathrm{~nm}$ & $6 \times 10^{8}$ \\
\hline $100 \mathrm{~nm}$ & $4 \times 10^{8}$ \\
\hline $1 \mu \mathrm{m}$ & $2 \times 10^{7}$ \\
\hline
\end{tabular}

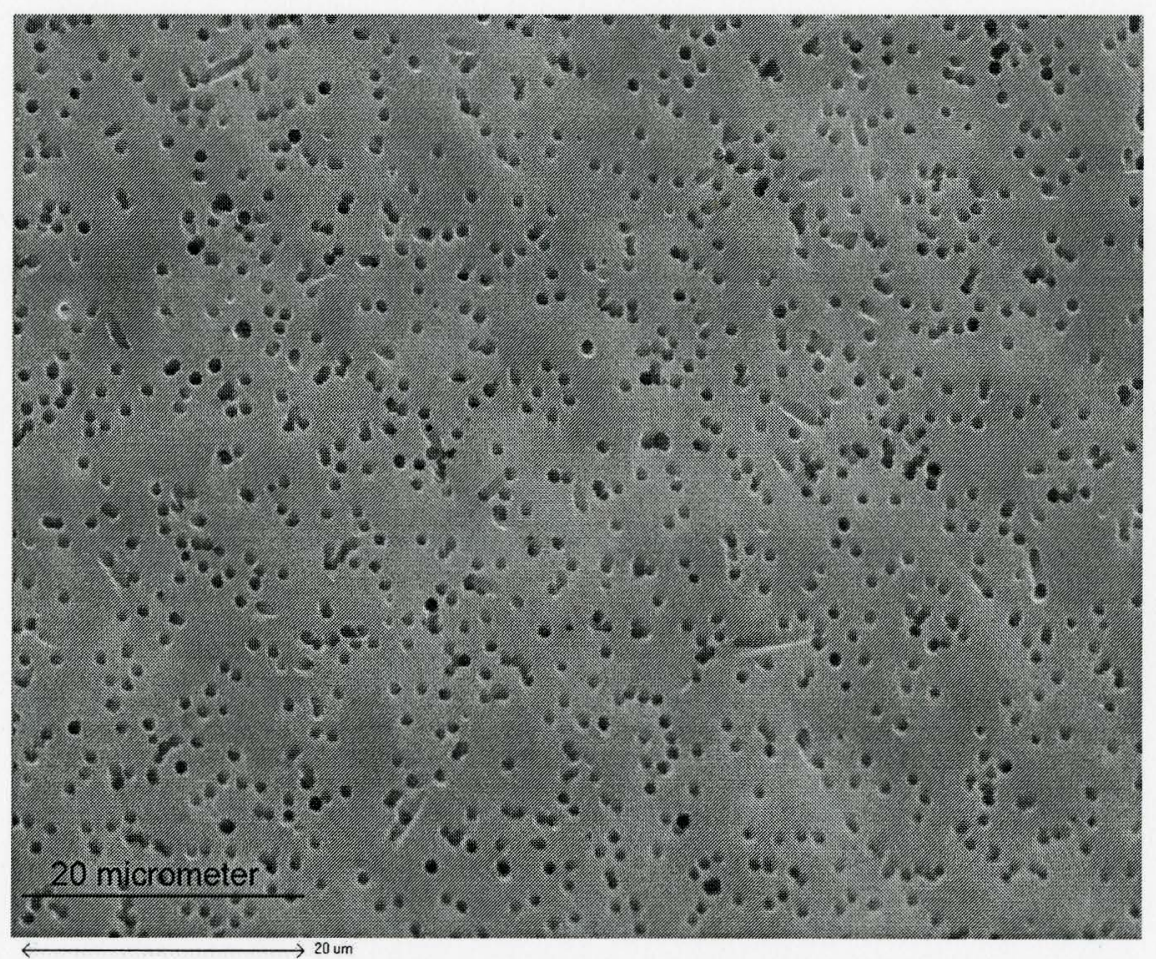

Figure 5.2. SEM image of nanoporous membrane: The pores are roughly round with an effective diameter of $1 \mu \mathrm{m}$. The pore density is $2 \times 10^{7}$. 


\subsubsection{Agar Gel}

Agar is extensively used to provide a solid surface for the growth of bacteria and fungi [Madigan 2005]. The basic agar after some modifications can be used to grow most of the microbes. For example, blood agar is obtained by adding sheep blood to agar gel to get desired environment for growing of the specific organism [Madigan 2005]. Agar is an unbranched polysaccharide obtained from the cell membranes of some species of red algae or seaweed. Agar consists of at least two separate polymers, agarose and agaropectin, however its actual structure is very complex [Lahaye 1991]. It has been shown that at least eleven different constituent structures could be identified in different agar bearing weeds depending on gender/species, environmental conditions and time of the year [Lahaye 1991].

Figure 5.3 shows the general molecular structure of the agar backbone. By varying $\mathrm{R}_{1}, \mathrm{R}_{2}$ (as shown in Figure 5.3, e.g. $\mathrm{CH}_{3}, \mathrm{C}_{2} \mathrm{H}_{5}$ ) and sulphur molecules, different types of agars are achieved [Lahaye 1991]. Variations in composition (e.g. gel concentration, gel type) lead to variations in physical properties (solidifying temperature, chemical composition, density, and color) of agar gel. But the working principle of the device is largely insensitive to these variations. Agarose, the gelling component of agar, has very similar properties as agar. In present devices it can be a substituted for agar.

Agar polymer, depending upon solution temperature, can have different structures. At higher temperatures, the polymer is dissolved in water as individual fibers, 
Master's Thesis-Sarvesh Upadhyaya-McMaster University-Mechanical Engineering

and as temperature decrease these fibers assemble to form large three dimensional network with wide pores ( 10-100nm) between them (Figure 5.4) [Lahaye 1991].

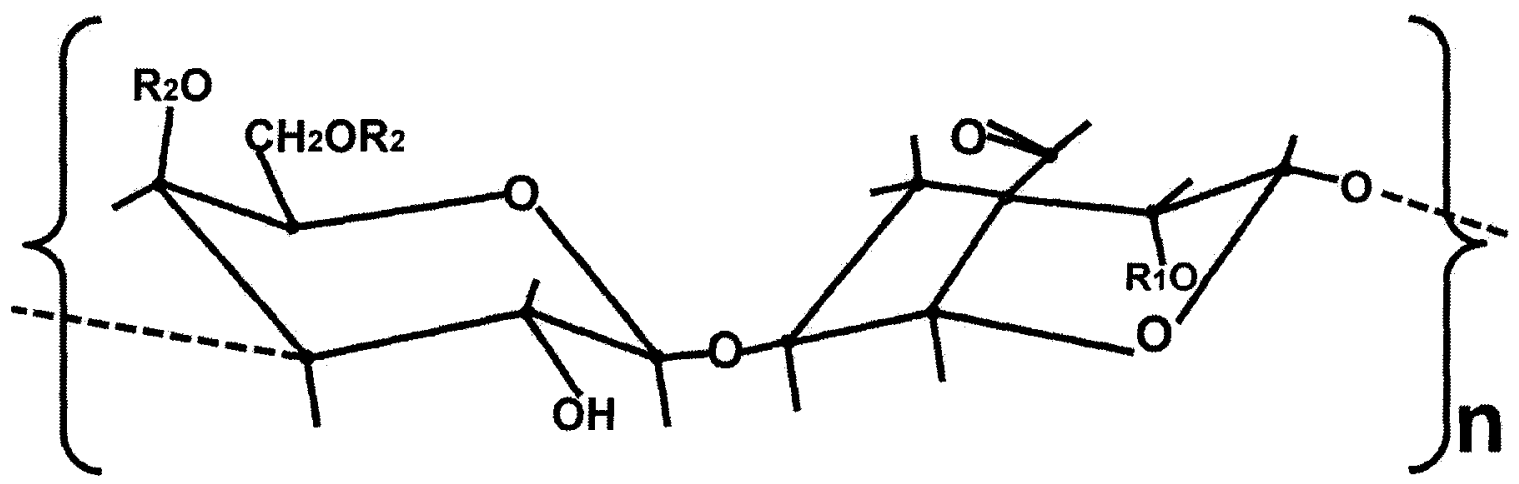

Figure 5.3. General molecular structure of agar backbone: By changing $R 1$ and $R 2$, different type of agar backbone can be obtained. Actual agar is a mixture of a number of such molecules.
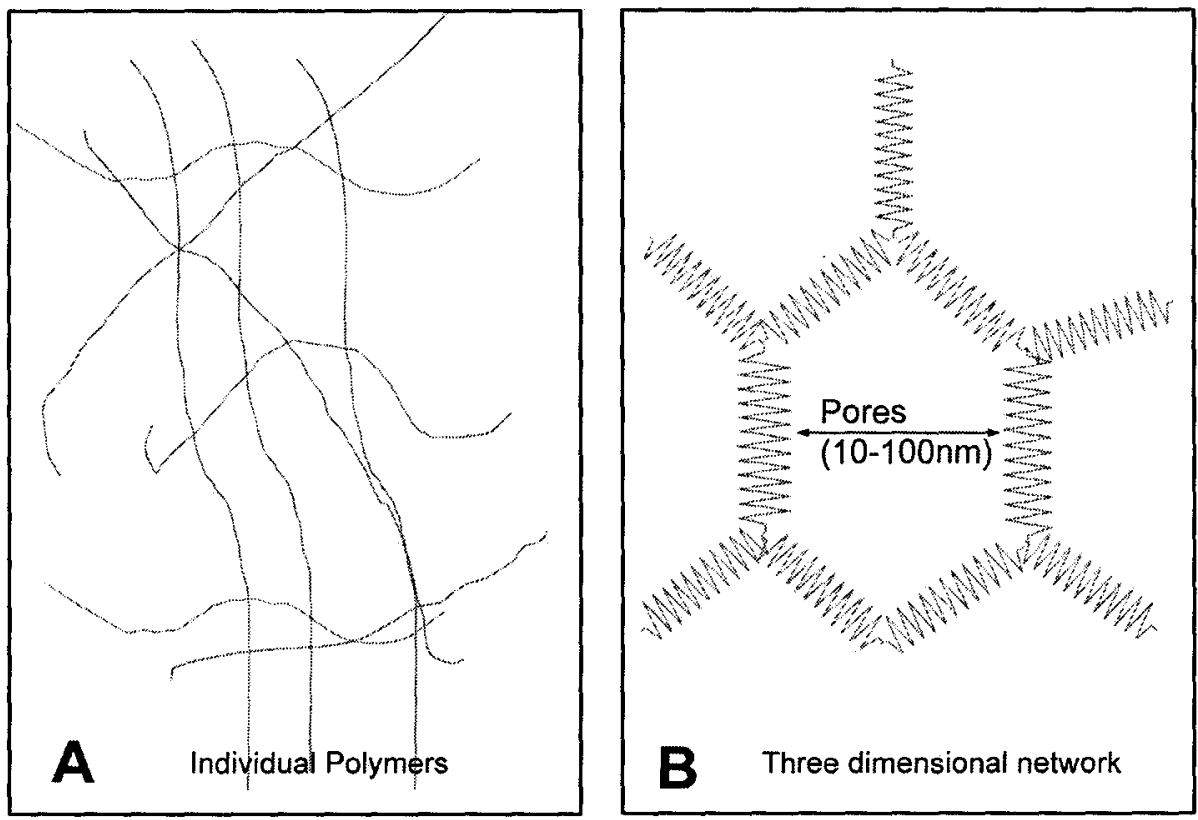

Figure 5.4 Gelling of agar: (A) At higher temperatures agar fibers are dissolved. (B) At lower temperatures, these fibers aggregate together to form a three dimensional network of pores. 
The pore size depends upon the gel type and the concentration and it ranges from $10 \mathrm{~nm}$ to $100 \mathrm{~nm}$ [Ackers 1962]. These pores are filled with a large amount of water, and this phenomenon is responsible for the ability of agar to hold large amounts of water. The size of the pores limits the size of molecule which can diffuse through it [Ackers 1962].

\subsection{FABRICATION PROCESS}

\subsubsection{Wafer Preparation}

A 3 inch silicon wafer (crystal orientation 100) supplied by University Wafers Corporation USA, is cleaned using acetone and distilled water. Then the wafer is plasma oxidized at $50 \mathrm{~W}$ rating for 30 seconds.

\subsubsection{SU8 Mold Fabrication}

All the parameters used are taken from the data supplied by manufacturer [Appendix 6]. SU8-100 is dispensed on the wafer and spun at 2750 RPM for 45 seconds producing a $100 \mu \mathrm{m}$ thick layer. Pre-exposure baking is done at $95^{\circ} \mathrm{C}$ for 1 hour to remove the excess solvent. The photo-mask is prepared using high quality printers on a transparency sheet. The drawings are prepared using AutoCAD software. The photoexposure (UV light, $600 \mathrm{~mJ} / \mathrm{cm}^{2}$ ) was done through a photo-mask for 60 seconds. Post exposure baking is done at $95^{\circ} \mathrm{C}$ for 10 minute by ramping the temperature slowly. Wafer is immersed in SU8 developer (mixture of ethyl lactate and diacetone alcohol) for 30 minutes. This process dissolves always the undeveloped SU8 leaving a negative replica of photomask made of SU8 on the silicon wafer. Isopropyl alcohol, which turns cloudy in 
presence of SU8, is used as a testing agent to verify its complete removal. After the development process, the mold is ready to be used. Process flow of SU8 mold fabrication process is given in appendix 1.

\subsubsection{PDMS Casting}

For fabricating the device, required amount of PDMS liquid is prepared by mixing 10:1 ratio of base and the curing agent. This liquid is casted on SU8 mold and heated at $65^{\circ} \mathrm{C}$ for 2 hours to cure it. After the curing process, the PDMS elastomer is peeled of the SU8 molds to obtain an inverse replica of the mold imprinted on the elastomer and is used to form the microfluidic network.

\subsubsection{Membrane Microcontact Printing}

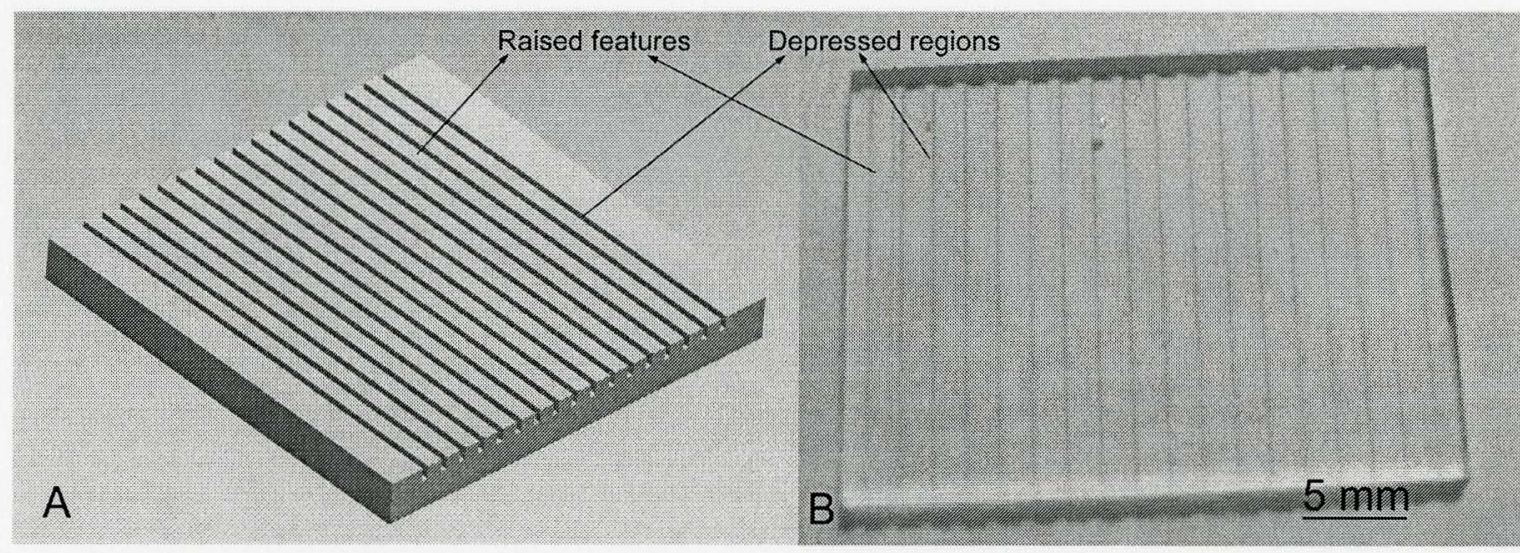

Figure 5.5 An example of PDMS sheet used for microcontact printing: Those areas of membrane touching raised regions in this sheet would get blocked. (A) 3D schematic. '(B). PDMS sheet. Different sheets are used for different membrane patterning.

Microcontact printing [Kumar 1994, Xia 1997] can also be called as a precise stamping method that can give submicron printing accuracies. This method is used to block pores in selected regions of nanoporous membrane. A PDMS microcontact stamp 
Master's Thesis-Sarvesh Upadhyaya-McMaster University-Mechanical Engineering

having features corresponding to desired pattern on membrane is fabricated using processes described in section 5.2.2 and 5.2.3. Figure 5.5 shows $3 \mathrm{D}$ schematic and picture of one such stamp used.

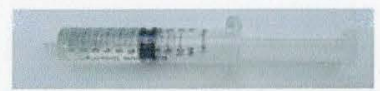

3:1 base to curing agent uncured PDMS. High curing agent gives thiner mixture.

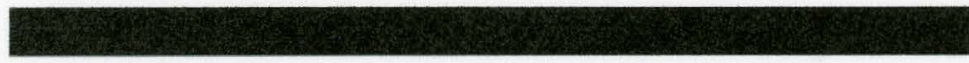

Cleaned 3 inch Si wafer

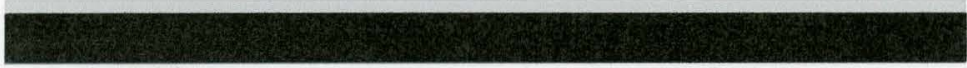

PDMS pored on wafer and spun at $10000 \mathrm{rpm}$ for 4 minutes to get thin PDMS layer

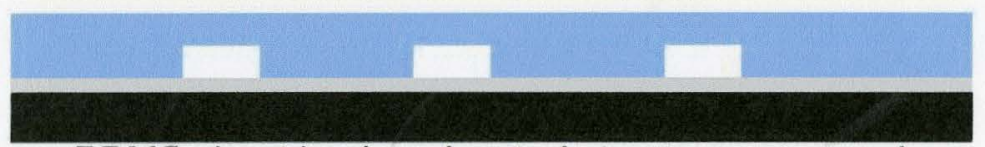

PDMS sheet having elevated structures stamped on wafer.

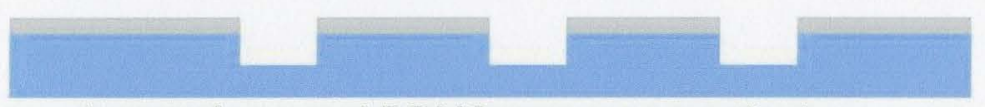

A part of uncured PDMS present on wafer is transfered to elevated features of PDMS sheet.

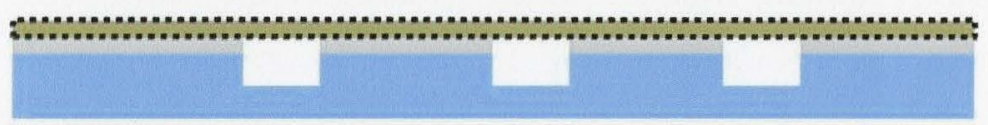

PDMS is stamped on nanoporous membrane transfering a part to uncured PDMS to membrane

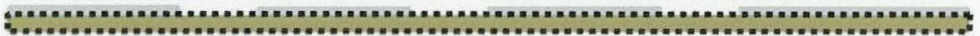

Membrane is peeled of from PDMS sheet, and

heated at $65 \mathrm{C}$ for 2 hours. The pores in selected regions are filled and blocked. Last three steps can be repeated mulitple times to get desired pattern.

Figure 5.6 Microcontact printing process flow: A thin layer of blocking agent (liquid PDMS) is spin coated on Si wafer. A stamp (PDMS sheet) is wetted on this wafer and then stamped on the nanopores membrane to block the pores in selected regions. 
3:1 base to curing agent PDMS liquid mixture is prepared and spin coated on a cleaned silicon wafer at $10000 \mathrm{rpm}$ for 4 minutes to get a thin layer of PDMS on top of the wafer. PDMS stamp (as shown in Figure 5.5) is then put on top of the wafer. This causes the sheet's elevated features to get wet with liquid PDMS present on wafer. The sheet was peeled off and stamped on the nanoporous membrane. The above process is repeated on the same membrane to get the desired pattern. Second time stamp microchannel direction is perpendicular that of first stamping. After two stamping steps only those pores, which are not blocked during both stamping steps, are left unblocked. Figure 5.6 shows the process flow for microcontact printing. Figure 5.7 shows one of patterned membranes. Process flow of polycarbonate membrane microcontact printing process is given in appendix 2 .

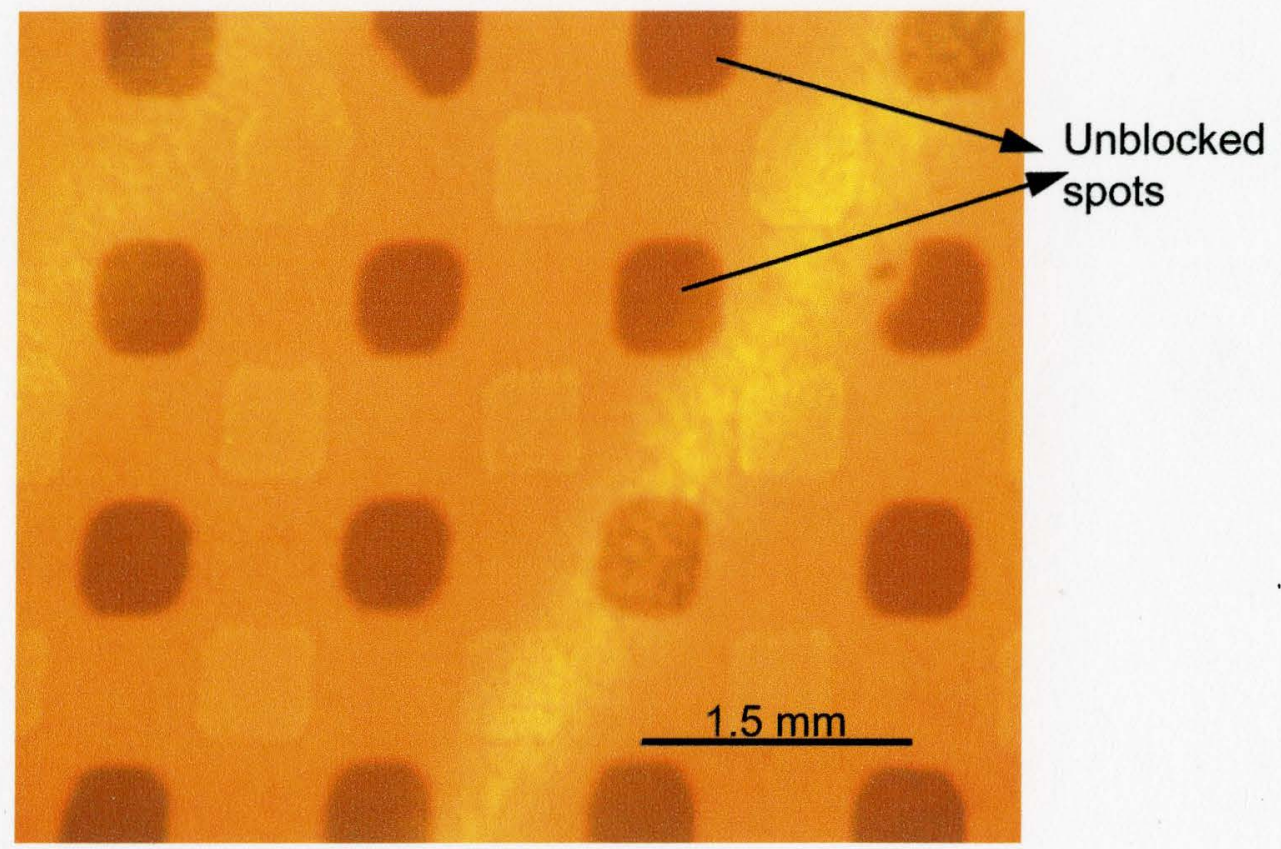

Figure 5.7 A patterned nanoporous membrane. 
Master's Thesis-Sarvesh Upadhyaya-McMaster University-Mechanical Engineering

\subsubsection{Device Assembly:}

PDMS sheet having microchannels (the bottom layer), the patterned membrane (middle layer), and top layer PDMS sheet (having cavity for containing gel) are stacked on top of each other and glued together. Uncured PDMS is used as glue. Molten gel (gel power mixed with water and boiled) is filled in the top layer cavity and solidified. The overall process flow for device fabrication is shown in Figure 5.8. Process flow of the device assembly process is shown in appendix 3 .
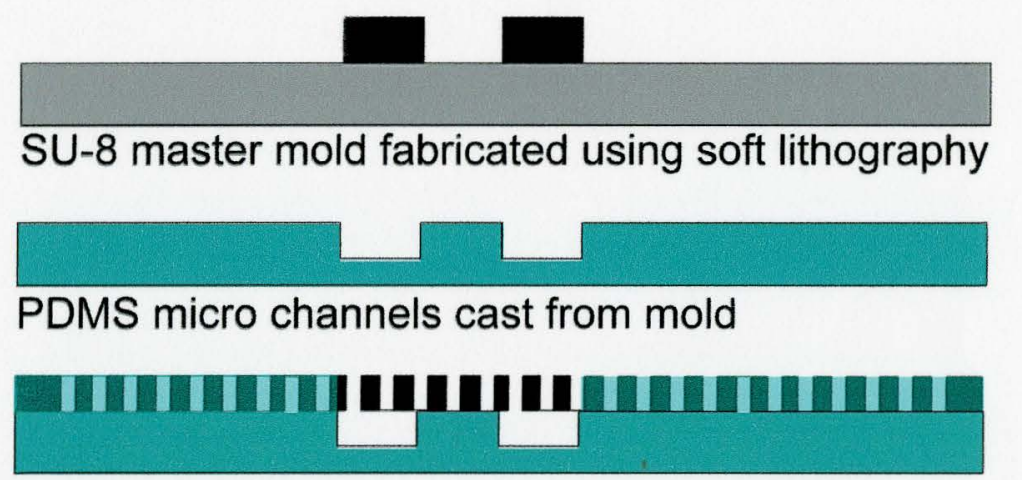

Bonding of nanoporous membrane and PDMS stamping for patterning of microinterface

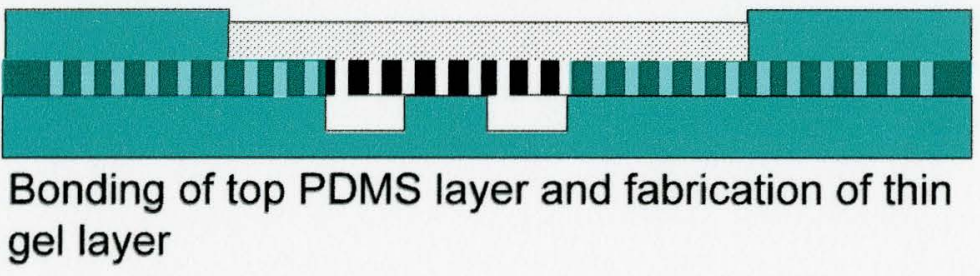

Figure 5.8 Device fabrication process flow: Three layers (bottom PDMS with microchannels, middle patterned membrane, top PDMS sheet with hole) were fabricated separately. These layers were stacked on top of one another and glued together. Gel was filled in top cavity as the last step. 
Master's Thesis-Sarvesh Upadhyaya-McMaster University-Mechanical Engineering

\subsection{EXPERIMENTAL SETUP}

Schematic of general experimental setup arrangement can be shown by Figure 5.9. There are some differences in experimental setups of individual devices and are described in respective chapters. The drugs are supplied into the microchannel using syringes. Electric voltage is supplied through two copper electrodes dipped into the drug in the reservoirs. Two separate sense lines are used to accurately measure voltage drop occurring in the membrane and gel. Both the electrodes being made of same material eliminates chances of development of net electrochemical potential.

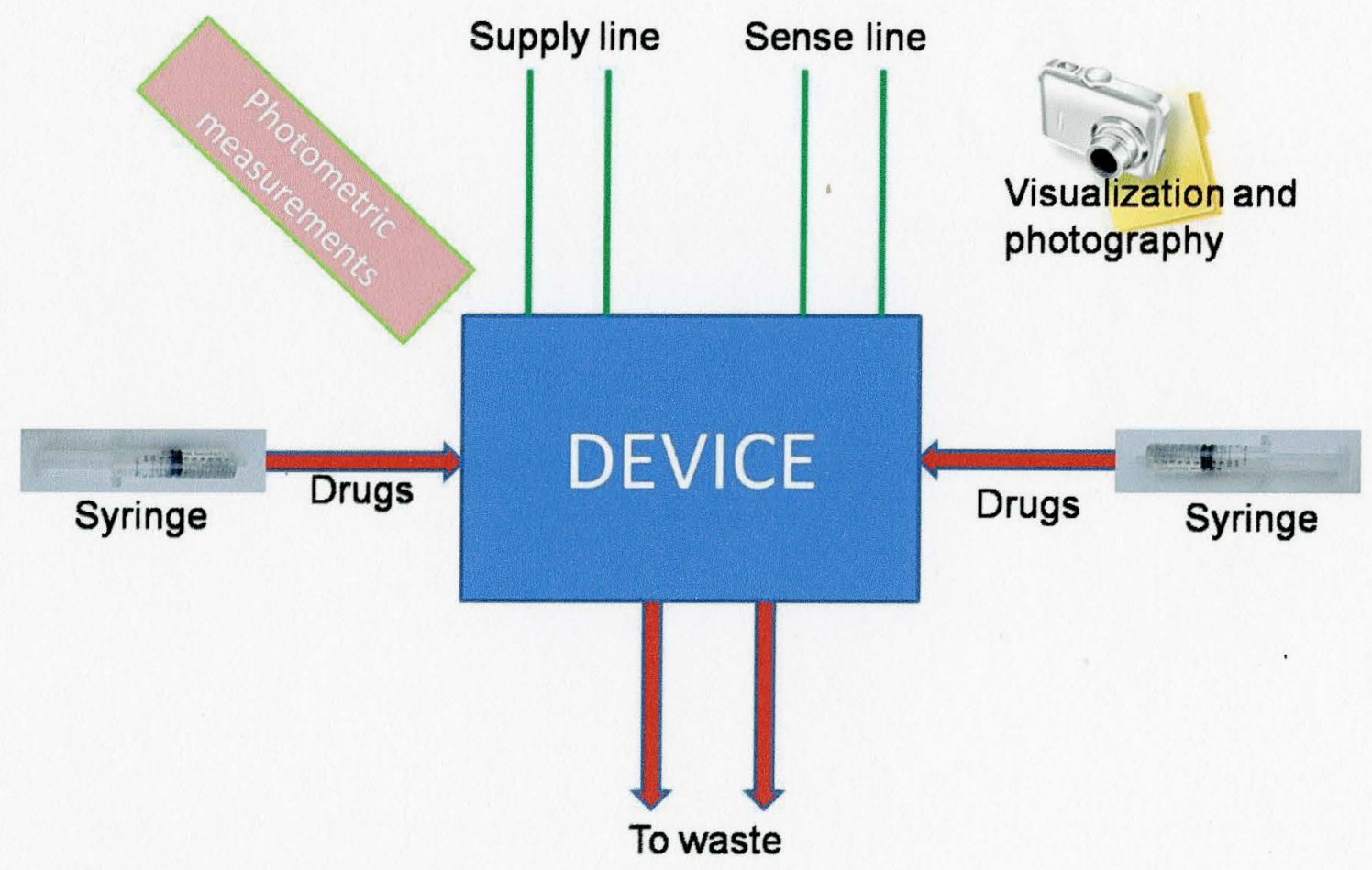

Figure 5.9 Schematic of general experimental setup. 


\subsection{WORKING MATERIALS}

The device is intended to be used in drug discovery applications hence the tested drugs/biomolecules should represent drugs or biomolecules that are typically expected to be used in this device. To cover the whole spectrum of typical molecules used in such applications following three types of compound are tested.

\subsubsection{Surrogate Drug Molecules}

Following criteria are used for selection of surrogate drug molecules to be tested in devices.

- The Lipinski's Rule of Five [Lipinski 1997]: It is a very popular method used in pharmaceutical industry to assess drug likeliness of any compound. According to this, for a compound to become drug, it should have not more than: 5 hydrogen bond donors, 10 hydrogen bond acceptors, 500 molecular weight and a Log P (a partition coefficient of water to oil solubility) of 5 .

- Colored: The drug should be bright in color so that it can be easily visualized and measured using photometric methods.

- Safe and Stable: It should be minimal toxic, stable, easy to handle and readily available.

Based on above criteria methylene blue and trypan blue are chosen as surrogate drug molecules. These are complex organic compounds having M.W. of 284.41and 872.9; $\log$ P: 1.16 and 4.05; H-bond donors: 0 and 8; H-bond accepters: 4 and 20 
respectively. These are purchased from Sigma Aldrich and used as such. The molecular charges on trypan blue and methylene blue are opposite in nature, and this would be used to verify electrophoretic nature of pumping mechanism.

\subsubsection{Proteins}

Bovine Serum Albumin (BSA) was selected as model protein as it is one of the widely used proteins in biological experimentations. It is easily available in large quantities (few grams) from Sigma Aldrich and can be easily visualized using protein staining methods. It is stored in refrigerator as suggested by the supplier.

\subsubsection{DNAs}

DNAs have become a molecule of immense importance in biological studies. Suitability of the device for using DNAs is demonstrated using oligomers (short length DNA having few base pairs to few tens of base pairs). The base pair sequence is selected to be random and $20 \mathrm{bp}$ long. The DNA (oligomer) is ordered from Mobix facility at McMaster University (http://www.science.mcmaster.ca/mobix/).

\subsection{Visualization}

Surrogate drugs (methylene blue and trypan blue) have strong colors and can be easily visualized. BSA is colorless (slightly grey) when dissolved in water, so it was visualized using SafeBlue ${ }^{\mathrm{TM}}$ Stain method of Invitrogen Inc. USA [http://www.invitrogen.com]. Details of this staining method are given in appendix 4 . DNA visualization is done with the help of ethidium bromide $(\mathrm{EtBr})$. EtBr when mixed with DNA bonds with the double helix backbone of DNA. After bonding it produces 
Master's Thesis-Sarvesh Upadhyaya-McMaster University-Mechanical Engineering

fluoresce with red-orange color in presence of UV light [Lakowicz 2006]. Details of EtBr DNA visualization are given in appendix 5.

\subsection{Measuring Instruments}

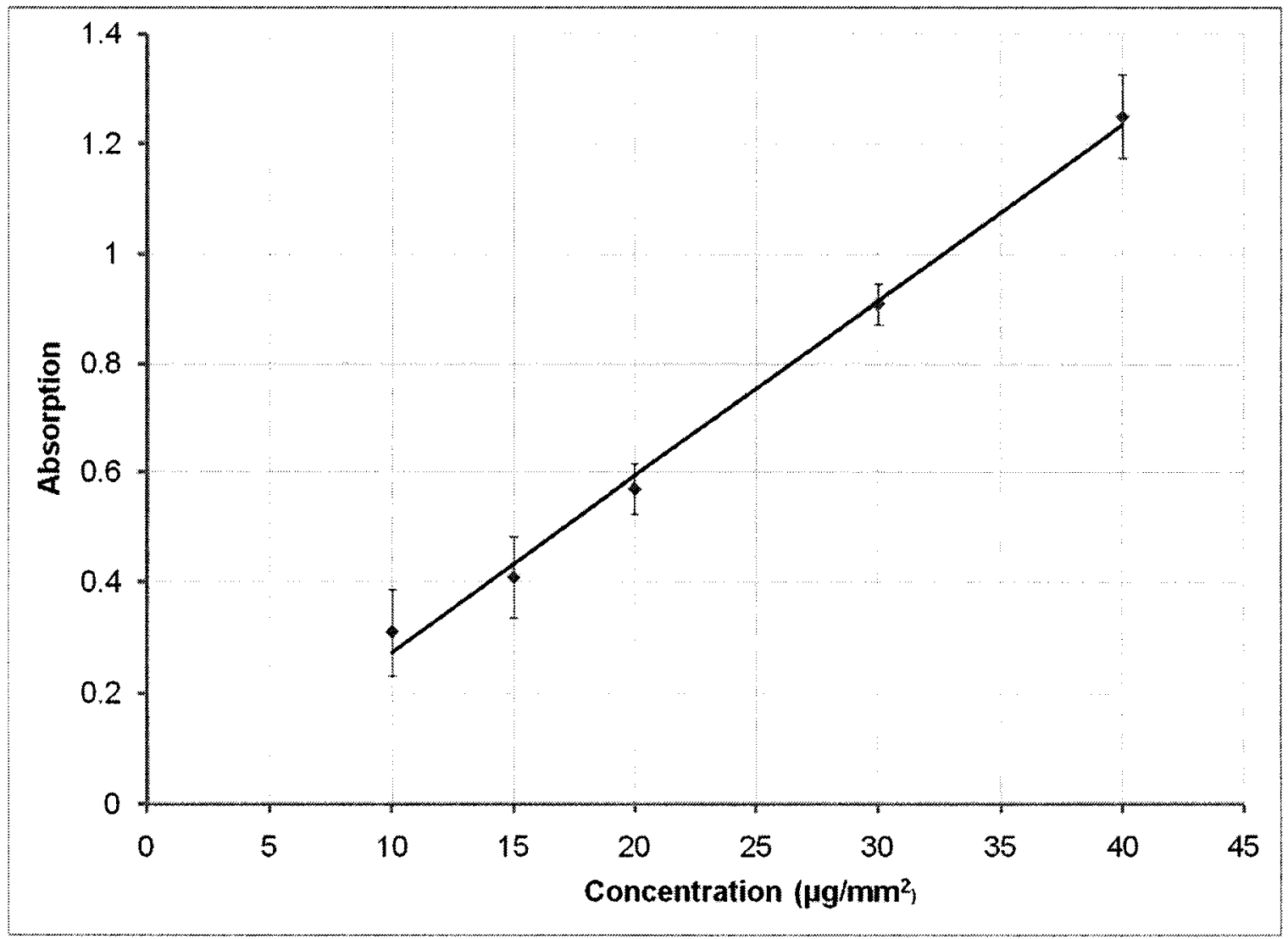

Figure 5.10 Calibration curve for trypan blue for photometric measurements: A number of $3 \mathrm{~mm}$ thick gel sheets having different known concentrations of trypan blue dies in them were run on Wallac 1420 VICTOR $2^{\mathrm{TM}}$ plate reader. Recorded absorption measurements were also corrected for background absorption by gel layer.

For quantitative measurement purpose photometric absorption method is used. Once drug molecules are pumped into the gel, the gel layer is separated from the device and concentration measurements are carried by Wallac 1420 VICTOR $2^{\mathrm{TM}}$ plate reader 
Master's Thesis--Sarvesh Upadhyaya-McMaster University-Mechanical Engineering

(PerkinElmer). A narrow beam of light ( $1 \mathrm{~mm}$ diameter) passes through the gel layer, and a part of it gets absorbed by the drug present in gel. The device was calibrated for trypan blue absorption using $3 \mathrm{~mm}$ thick gel sheets ( $2 \%$ gel content) having different known concentrations of trypan blue mixed in the gel. The Figure 5.10 shows the calibration curve. Recorded absorption measurements were also corrected for background absorption by gel layer. The minimum detection limit of the method was calculated based on 7 measurements and was found to be $4.2 \mu \mathrm{g} / \mathrm{mm}^{2}$.

Based on above fabrication method, a number of devices are fabricated. These devices are divided in three groups, single spot forming devices, multiple spot forming devices. Fabrication and testing of these devices are discussed in coming chapters. 
Master's Thesis - Sarvesh Upadhyaya - McMaster University - Mechanical Engineering

\section{CHAPTER 6}

\section{SINGLE DRUG SPOT DEVICES}

This chapter deals with the design, fabrication, and testing of single drug spot forming devices (the device able to make only one drug spot in an agar gel slab). These devices incorporate all the essential features of the cell based HTS system in a simple design layout and are most suitable for demonstrating and characterizing the working of the device.

\subsection{DEVICE DESIGN}

The 3D schematic and typical dimensions of single spot forming device are shown in Figure $6.1 \mathrm{~A}$ and $6.1 \mathrm{~B}$ respectively. A number of single spot devices were designed by varying the dimensions of the microchannel width and gap between microchannels. The bottom layer consists of two parallel microchannels, which have reservoirs at the ends. The width of the microchannel is varied between $200 \mu \mathrm{m}$ to $500 \mu \mathrm{m}$, while the gap between microchannels is changed between $2 \mathrm{~mm}$ to $5 \mathrm{~mm}$ ). The open pore patch dimension is either $500 \mu \mathrm{m} \times 500 \mu \mathrm{m}$ or $200 \mu \mathrm{m} \times 200 \mu \mathrm{m}$. 
Master's Thesis - Sarvesh Upadhyaya - McMaster University - Mechanical Engineering
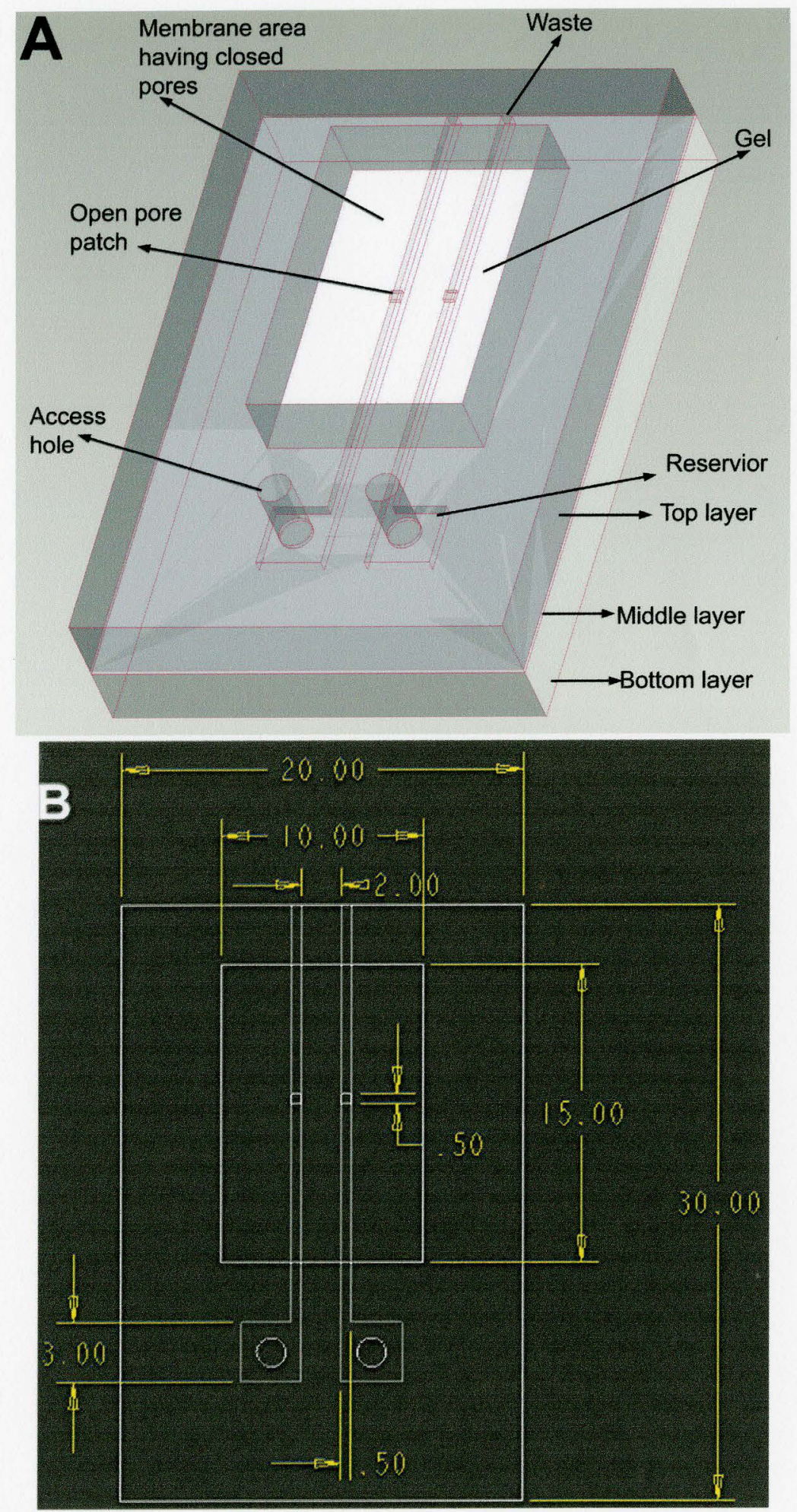

Figure 6.1 Single spot forming device design: (A). 3D schematic. (B)Typical dimensions 
Master's Thesis - Sarvesh Upadhyaya - McMaster University - Mechanical Engineering

\subsection{FABRICATION PROCESS}

All the devices considered in this chapter are fabricated using the method described earlier, as in section 5.2. Fabrication is performed using an SU8 mold on which PDMS is casted to embed microchannels into a $3 \mathrm{~mm}$ thick PDMS sheet. Membrane microcontact printing is done using the method described in section 5.2.4. After microcontact printing, all the pores except those of the two square areas of $500 \mu \mathrm{m} \times 500 \mu \mathrm{m}$ (or $200 \mu \mathrm{m} \times 200 \mu \mathrm{m}$ ) on membrane are blocked. Top layer of these devices is a PDMS layer with $10 \mathrm{~mm} \times 15 \mathrm{~mm}$ hole, which is used for filling the gel after assembly process.

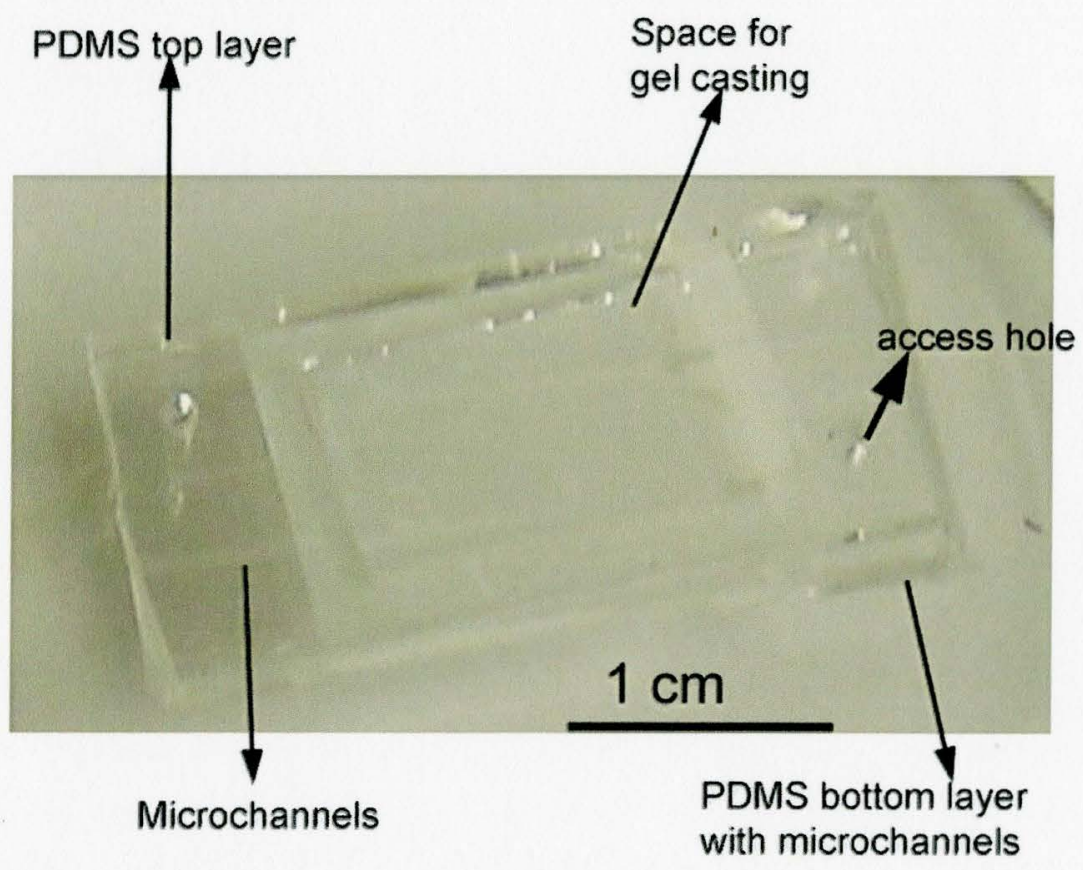

Figure 6.2 One of the single spot devices: Three layered device (middle membrane layer is thin and transparent, hence not visible). Gel is casted in the device just before experimentation. 
Master's Thesis - Sarvesh Upadhyaya - McMaster University - Mechanical Engineering

Assembly of the devices are done using the method described in section 5.2.5. During the assembly process, open pore patches on membrane are aligned with bottom PDMS layer microchannels. The top PDMS layer hole is filled with agar gel (1-3\%).

\subsection{FABRICATED DEVICES AND EXPERIMENTAL SETUP}

A number of devices based on above designs and fabrication techniques are made.

Figure 6.2 shows one such fabricated device. Out of the constructed three layers, top and bottom layers are visible, while the middle layer which is a very thin transparent membrane is not clearly visible.

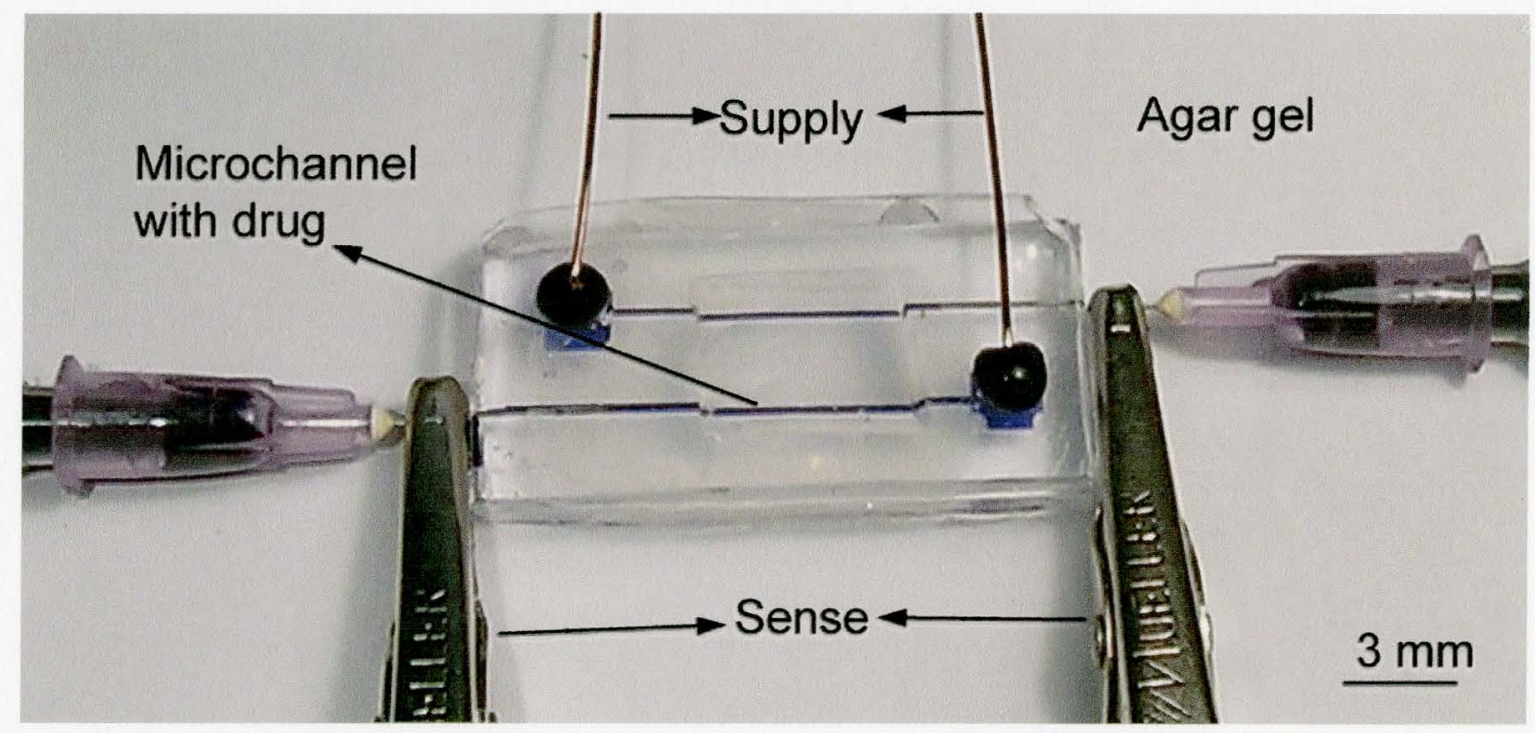

Figure 6.3 Single spot forming device experimental setup: Syringes are used for drug supply in microchannels. Same material (copper) electrodes are used for potential supply. Separate sense lines are used for potential measurements. Quantitative measurements are done on a separate instrument by taking out gel from the device.

The experimental setup for working demonstration and characterization is shown in Figure 6.3. Testing compound, trypan blue in this case, is pumped into microchannels 
Master's Thesis - Sarvesh Upadhyaya - McMaster University - Mechanical Engineering

using syringes. Electric voltage is supplied through two copper electrodes dipped into the drug in the reservoirs. Two separate sense lines are used to compensate for voltage drop occurring in microchannels. Both the electrodes are made of the same material (copper) to eliminate any chances of net electrochemical potential development.

\subsection{PUMPING DEMONSTRATION}

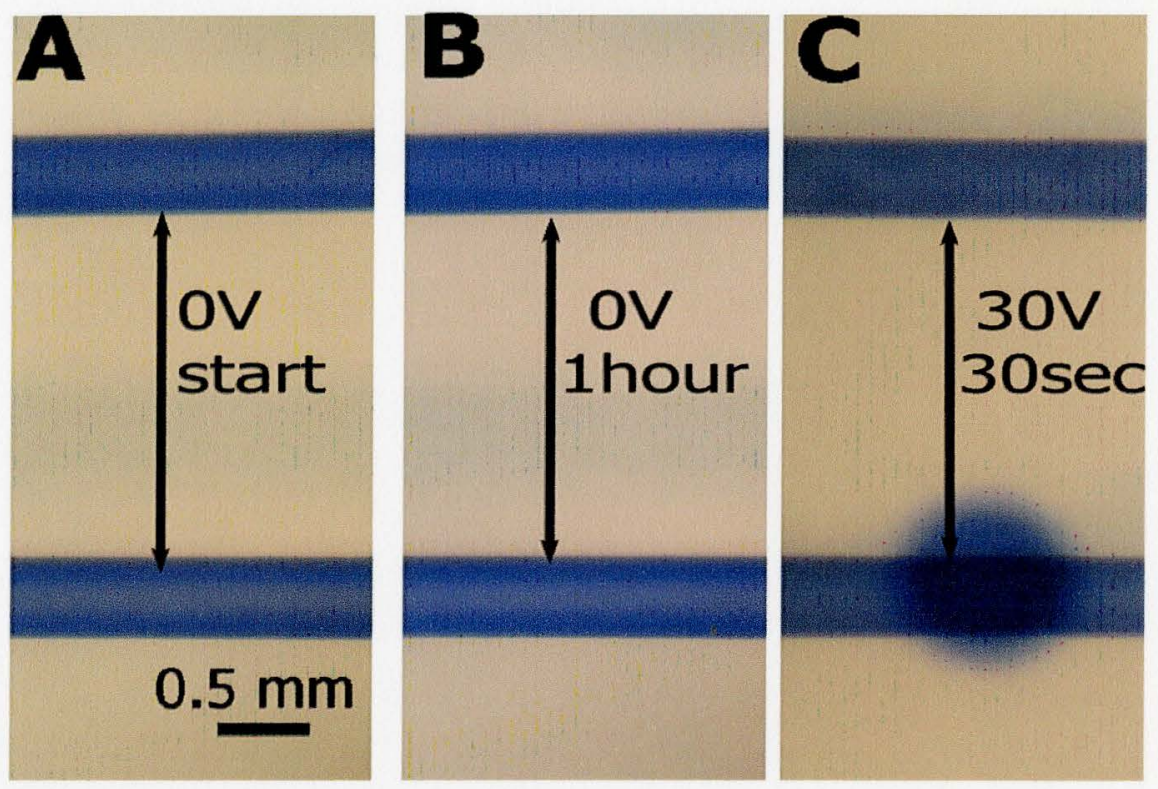

Figure 6.4 Drug pumping demonstration: Two microchannels separated by $2 \mathrm{~mm}$ gap and filled with trypan blue. The membrane being transparent is not visible in the figure. A: No voltage at time zero. B. No voltage till time 1 hour. No visible diffusion of drug form microchannel to agar layer. C. $30 \mathrm{~V}$ for $30 \mathrm{sec}$ pumping of drug from microchannel to gels takes place.

The first experiment demonstrated here is the device capability to pump drug in a controlled fashion. For this purpose, one of the above mentioned devices is run using trypan blue as surrogate drug. The microchannels are filled with trypan blue. Trypan blue is allowed to diffuse through the nanoporous membrane for 1 hour. The pore size is 
Master's Thesis - Sarvesh Upadhyaya - McMaster University - Mechanical Engineering

$100 \mathrm{~nm}$, microchannel width is $0.5 \mathrm{~mm}$ and the gap between microchannels is $2 \mathrm{~mm}$. There is no diffusional transport (less than visual and detection limit i.e. 4.2 micrograms $/ \mathrm{mm}^{2}$ ) through the nanoporous interface in the absence of electric field even after one hour of establishment of the concentration gradient (between gel and microchannel) as is seen in Figure 6.4B. But the application of electric potential for 30 seconds creates electrokinetic transport of drug molecule into the gel as shown in Fig 6.4C.

Based on this experiment it can be concluded that the device can pump the drug from microchannel into the gel in a spot form and there is no drug diffusion (negligible) from microchannel to gel, when electric field is not applied. Hence the drugs can be pumped (or not pumped) in the agar gel from microchannel as required.

\subsection{PUMPING MECHANISM}

It has been shown by previous works that, actual pumping mechanism is complex in nature and consists of electrophoretic (see section 4.3.1.1) and electroosmotic (see section 4.3.1.2) and diffusional (section 4.3.1.3) flows and depends upon a number of parameters (e.g. electric field, solute ions, concentrations, solution $\mathrm{pH}$, pore size, pore density, molecular size and molecular charge) [Kemery 1998]. As shown in Figure 6.4 diffusional transport is negligible. This result is in agreement with results obtained by Kuo et. al. [Kuo 2003] when they did not observe any significant diffusion in 10 minute duration using $200 \mathrm{~nm}$ and $15 \mathrm{~nm}$ pore sizes. To find out the dominant mode of pumping between electrophoresis and electroosmosis, two oppositely charged molecules, trypan 
Master's Thesis - Sarvesh Upadhyaya - McMaster University - Mechanical Engineering

blue (negatively charged) and methylene blue (positively charged) are pumped in identical conditions ( $2 \%$ agar gel, applied voltage $30 \mathrm{~V}$ for 30 seconds). Upon application of electric potential both microchannels have opposite potentials. Trypan blue was pumped from negative microchannel while methylene blue was pumped from positive microchannel. Moreover, in the gel both dyes moved in opposite directions. In electrophoresis oppositely charged molecules are expected to move in opposite directions, hence opposite direction motion of trypan blue and methylene blue proves the dominant mechanism of pumping by the membrane to be electrophoresis. If electroosmosis was the dominant mode of pumping, both the dyes would have got pumped from same microchannel. This is so because in electroosmosis the pumping is dependent on zeta potential and applied electric field and pumping direction would not change due to any changes in solute molecular charges.

\subsection{POST PUMPING DRUG DIFFUSION}

For satisfactory functioning of these devices, the drugs infused into the gel from its bottom surface should reach the cells which will be grown on the top surface through diffusion (refer section 4.3.3 for details). To verify this, post pumping diffusional behavior of the device is studied. First, pumping was carried out for $3 \mathrm{~min}$ (at 30V) and then switched off. Gel was left undisturbed in the device and photographs were taken at various time intervals. As can be seen in Figure 6.5, the drug spot size grows with passage of time. More ever, it can also be observed in the pictures that, with time the 
Master's Thesis - Sarvesh Upadhyaya - McMaster University - Mechanical Engineering

dye concentration at the spot boundaries become blurry (a characteristic of molecular diffusion).

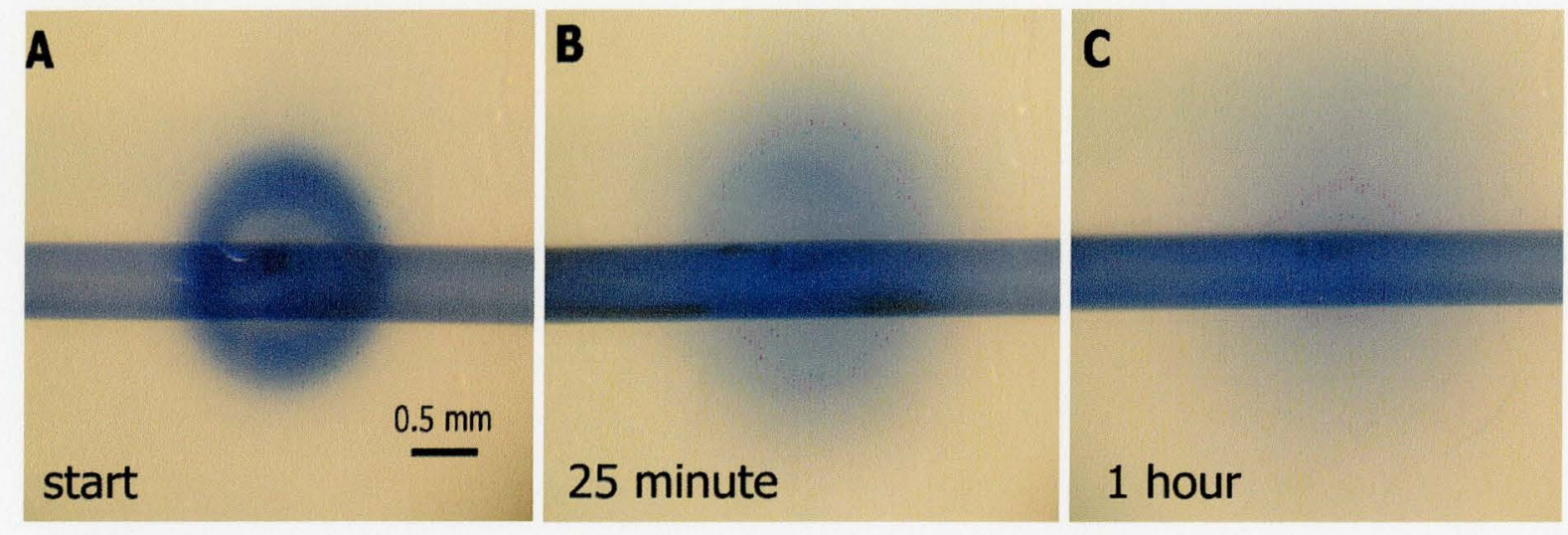

Figure 6.5 Post pumping drug diffusional behavior (qualitative): Drug molecules diffuse through the gel causing the spot size to grow. The diffusion rate (hence spot size growth rate) depends upon gel concentration and drug molecule size.

To corroborate qualitative information of Figure 6.5 , the above experiment is run again with quantitative measurements. The measurement method used is photometric (see section 5.6 for details). Results are shown in Figure 6.6. At the end of active pumping the concentration profile (concentration vs. distance from centre of the spot) is of bell curve shape. Concentration at centre is high and spot boundaries are sharper (high concentration gradients i.e. steeper graph). The spot size is $1.8 \mathrm{~mm}$ in radius. After one hour, the spot size increases (radius $3.8 \mathrm{~mm}$ ) and concentration gradient at the boundaries decreases (i.e. flatter graph). 
Master's Thesis - Sarvesh Upadhyaya - McMaster University - Mechanical Engineering

Based on above experiments it is concluded that the drug diffuses in gel slab and spot size increases with passes of time while concentration decreases. Hence the drug can reach up to the top gel surface.

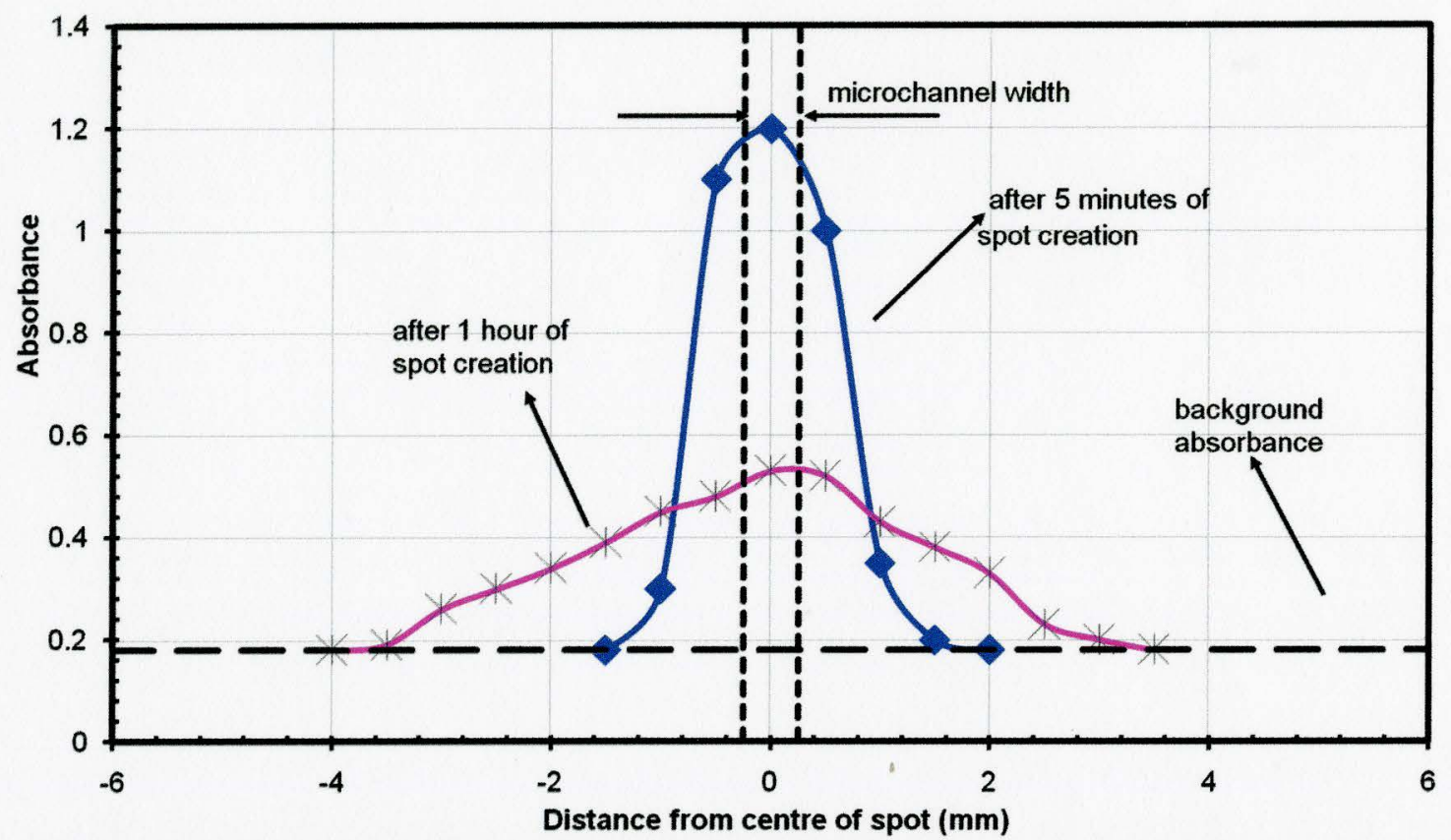

Figure 6.6 Post pumping drug diffusional behavior (quantitative): Smaller spot $(1.8 \mathrm{~mm})$ with sharper boundaries (steeper line). With passes of time spot size grows $(3.8 \mathrm{~mm})$ and concentration profile becomes flatter (a characteristic of diffusion)

\subsection{DEVICE CHARACTERIZATION}

\subsubsection{Spot Radius Growth Rate}

Spot radius growth happening in the device (i.e. increasing radius of drug spot in gel vs. time) is of two types. The first type of growth is observed when electric field is 
Master's Thesis - Sarvesh Upadhyaya - McMaster University - Mechanical Engineering

present (i.e. during pumping), during which higher concentration gradients (C.G.) are observed, can be calculated from following equations

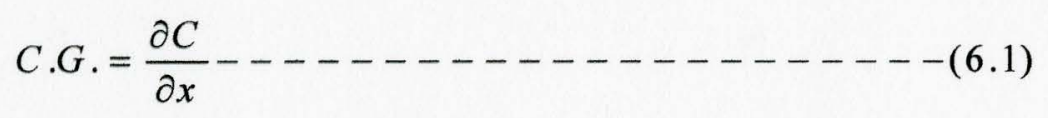

Where $\mathrm{C}$ is drug concentration and $\mathrm{x}$ represents length.

Average concentration gradient across a drug spot can be given by following equation,

$C \cdot G .=\left(C_{2}-C_{1}\right) / r-\cdots-\cdots-\cdots-\cdots(6.2)$

Where $\mathrm{C}_{2}$ and $\mathrm{C}_{1}$ are the drug concentration just outside the drug spot and at the centre of drug spot respectively and ' $r$ ' is drug spot radius.
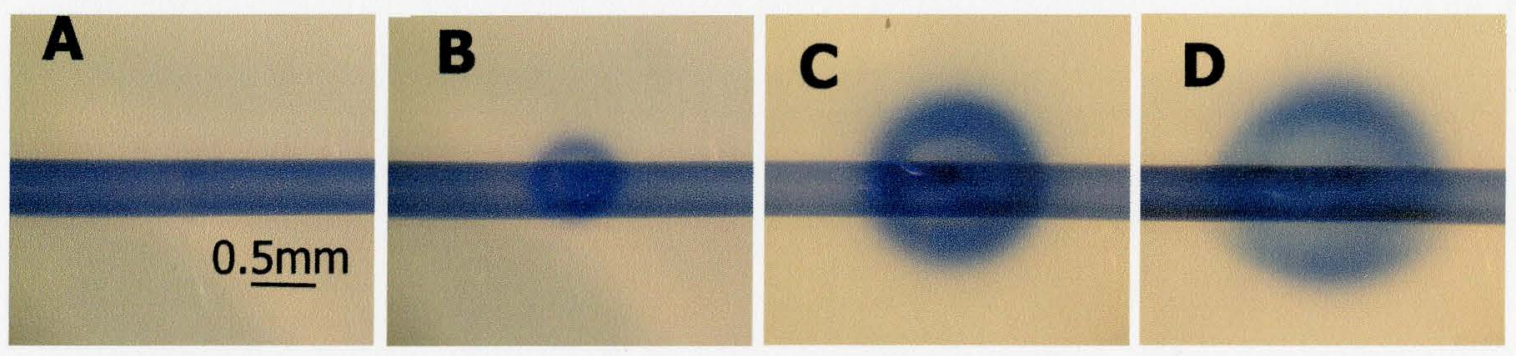

Figure 6.7 Drug spot radius growth during pumping (qualitative): Spot size growth with duration of applied electric potential (30V) application. A. no potential. B. $10 \mathrm{sec}$ C. $30 \mathrm{sec}$ D. 3 min.

At the beginning of pumping, $r$ is small hence higher gradients are present. More ever, during the pumping period electrophoretic migration of drug molecules also happens causing higher radius growth rate. To measure the radius growth rate during 
Master's Thesis - Sarvesh Upadhyaya - McMaster University - Mechanical Engineering

pumping, parameters other than time (such as voltage- $30 \mathrm{~V}$, pore size $100 \mathrm{~nm}$, trypan blue as model dye, gel concentration- 2\%) are kept constant. As qualitatively shown in Figure 6.7 , the size of the spot increased with time during pumping. The spot grew from $0.5 \mathrm{~mm}$ (the unblocked pore patch size) to $2.5 \mathrm{~mm}$ in $3 \mathrm{~min}$.

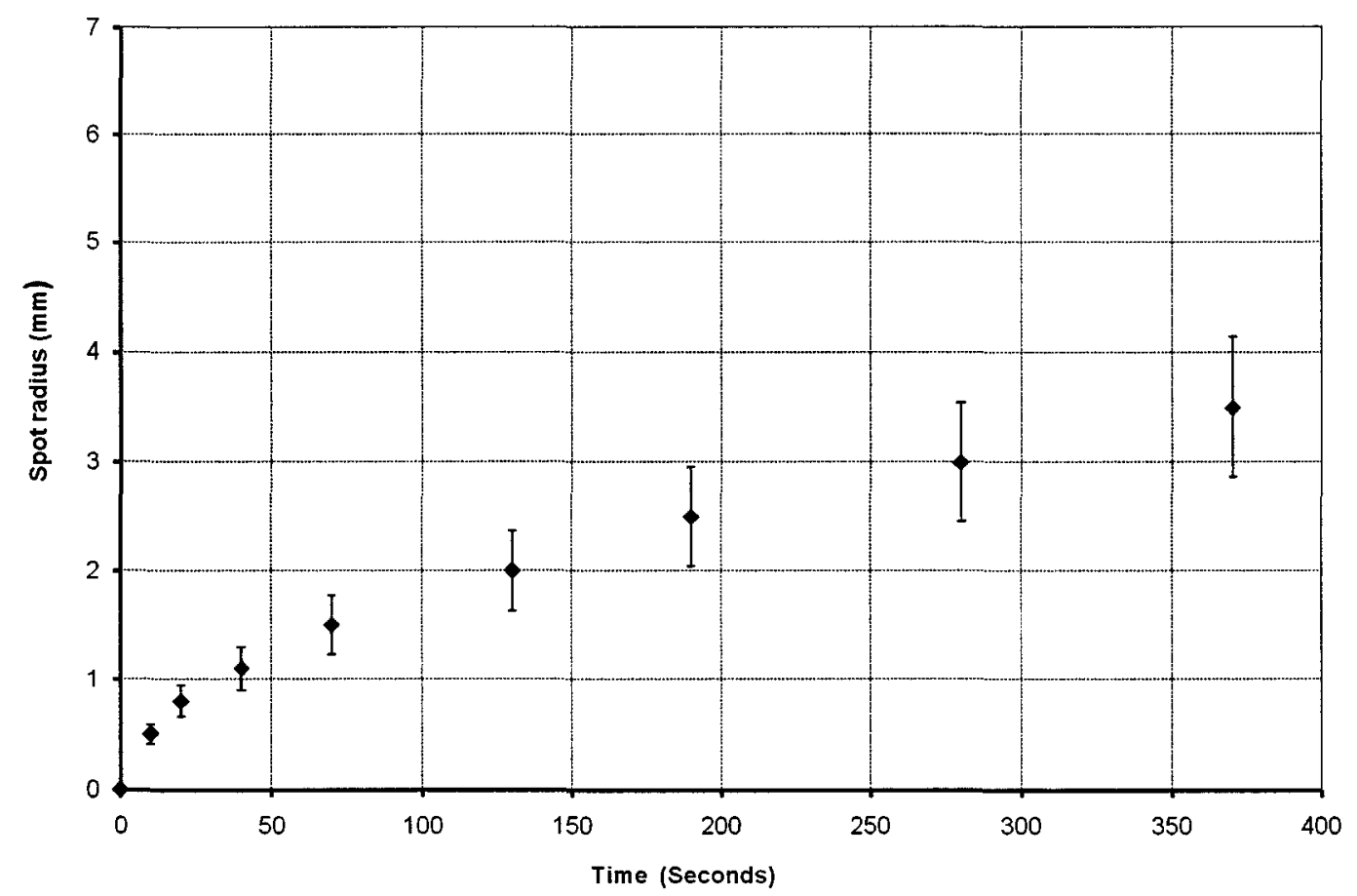

Figure 6.8 Drug spot radius growth during pumping (quantitative): Spot size growth with for constant operating conditions (voltage-30, pore size $100 \mathrm{~nm}$, model dye, gel concentration-2\%)

Quantitative growth rate measurements are done using photometric method. In this measurement there was a delay of one minute between spot formation and spot radium measurements (due to time required to chance the gel form the device to 
Master's Thesis - Sarvesh Upadhyaya - McMaster University - Mechanical Engineering

measuring instrument). Figure 6.8 shows the spot radius growth with respect to time. Spots grow faster initially then slow down.

Quantitative post pumping radius growth rate measurements are done using photometric method. Figure 6.9 shows the radius growth with time. As expected, the growth rate is much slower as compared to that of during pumping.

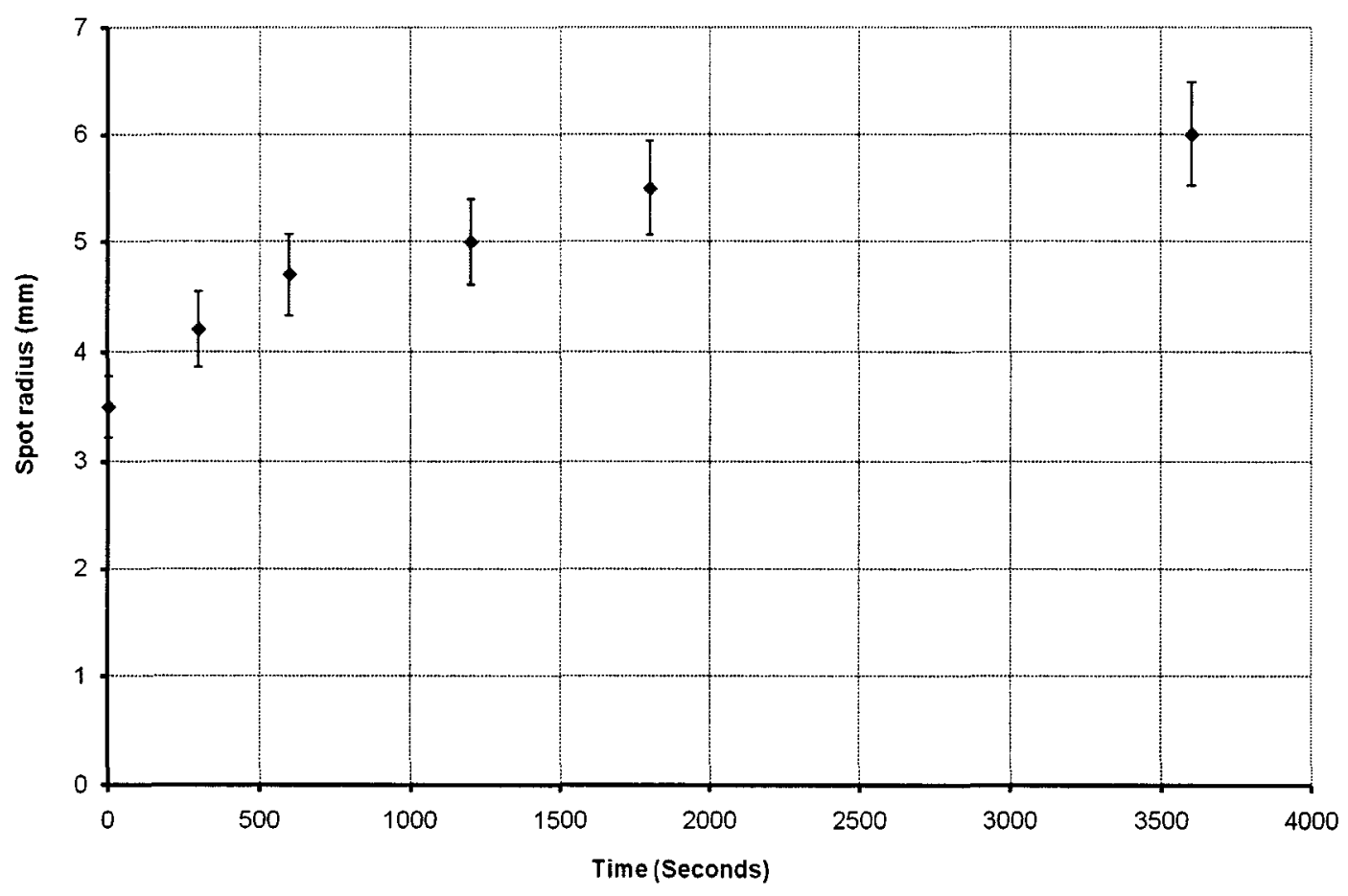

Figure 6.9 Drug spot radius growth after pumping (quantitative): Spot size growth due to diffusional effects. Initially growth is faster then it slows down with increasing spot size (a characteristic of diffusional migration).

\subsubsection{Drug Dose Control}

The amount of drug that gets pumped depends upon a number of operating parameters like time of pumping, voltage applied, pore size etc. Out of these, the simplest 
Master's Thesis - Sarvesh Upadhyaya - McMaster University - Mechanical Engineering

parameter to control is time, as other parameters (pore size, density etc) cannot be changed once device has been fabricated.

For a fixed operating condition (i.e. voltage-30, pore size $100 \mathrm{~nm}$, trypan blue as model dye, gel concentration- 2\%) the amount of drug pumped was measured for various time instances using photometric measurements. Figure 6.10 shows the results obtained. As expected, a linear relationship between time duration and dosage is observed.

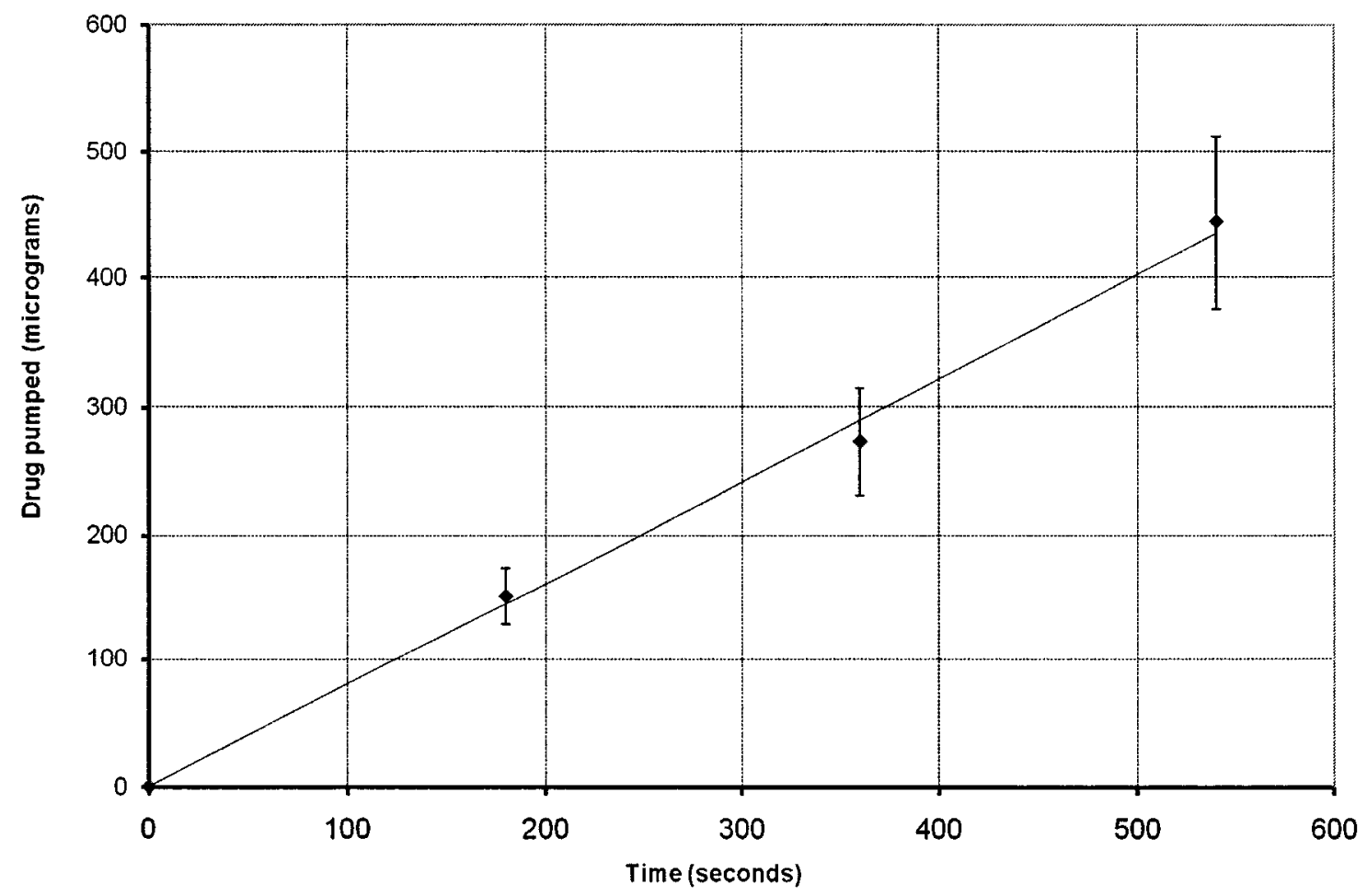

Figure 6.10 Drug dose vs. time: The amount of drug pumped during different times at constant operating conditions (voltage-30, pore size $100 \mathrm{~nm}$, trypan blue as model dye, gel concentration- 2\%). 
Master's Thesis - Sarvesh Upadhyaya - McMaster University - Mechanical Engineering

\subsubsection{Current and Heat Generation}

Energy consumed by the device is too small $(<1$ Watts) to be considered for calculating the economic value of electric power consumption. But the heat generated due to the current flow is calculated (based on voltage drop and current flow) to evaluate any chances of gel melting due to elevated temperatures. Actual magnitude of current depends upon a number of parameters like voltage, ion concentration, pore size and density etc. Current for some representative cases $(0.5 \mathrm{M} \mathrm{KCl}$, single spot, open pore patch size $0.5 \mathrm{~mm} \times 0.5 \mathrm{~mm}$ ) are measured and the values are shown in Table 6.1.

Table 6.1: Current and heat generation in a typical operating condition

\begin{tabular}{|l|l|l|l|}
\hline Voltage & Current & $\begin{array}{l}\text { Heat Generation } \\
\text { rate }\end{array}$ & $\begin{array}{l}\text { Gel slab }(3 \mathrm{~mm} \times 10 \mathrm{~mm} \times 15 \mathrm{~mm}) \\
\text { temperature rise in one minute } \\
\text { (without considering heat losses) }\end{array}$ \\
\hline 5 & 0.00021 & 0.00105 & $\left({ }^{\circ} \mathrm{C}\right)$ \\
\hline 15 & 0.00059 & 0.00885 & 0.033 \\
\hline 30 & 0.0011 & 0.033 & 0.28 \\
\hline 60 & 0.00228 & 0.1368 & 1.04 \\
\hline
\end{tabular}


Master's Thesis - Sarvesh Upadhyaya - McMaster University - Mechanical Engineering

The calculated value of heat generated is also given in table 6.1. Expected temperature rise of gel slab $(3 \mathrm{~mm} \times 10 \mathrm{~mm} \times 15 \mathrm{~mm})$ due to this heat addition is also calculated by assuming that there is insignificant heat loss from gel slab to surrounding. In reality due to the small size of the device $(<3 \mathrm{~cm})$ this heat loss would be significant (higher conductive heat losses at smaller length scales). But this assumption is made in order to simplify the analysis and to make sure that in no circumstance the actual temperature rise is more than the calculated one. It is also assumed that all the electrical heat generated is uniformly retained by agar gel. Specific heat of gel is taken to be equal to that of water $\left(4.186\right.$ joule/gram $\left.{ }^{\circ} \mathrm{C}\right)$. The maximum temperature rise is calculated to be $4.36^{\circ} \mathrm{C}$. This value is an order of magnitude lesser than the temperature rise of $65^{\circ} \mathrm{C}$ required to melt the gel (assuming room temperature to be $25{ }^{\circ} \mathrm{C}$ and gel melting temperature to be $90{ }^{\circ} \mathrm{C}$ ). From photographs of the gel taken after pumping no sign of gel melting is observed.

In the device discussed in this chapter, it has been demonstrated that drugs can be pumped locally forming a drug spot in agar gel. Device characterization like drug spot radius growth rates and doze control has been performed. Next chapter deals with multispot forming device and would involve biomolecule pumping and microarray formation. 


\section{CHAPTER 7}

\section{MULTIPLE DRUG SPOT DEVICES}

These devices are more complex as compared to single spot forming devices and can produce more than one drug spot in a single run. These are used to demonstrate the array forming capabilities. Design, fabrication and testing of two such devices are described in this chapter.

\subsection{Device Designs}

Design of two multi spot forming devices (three spot and microarray) is described below.

\subsubsection{Three Spot Device}

This design is used to test the ability of electrically controlled independent spot creation. The 3D schematic and typical dimensions of the device are shown in Figure 7.1A and 7.1B respectively. It has three microchannels surrounding a fourth microchannel. This arrangement, as shown in Figure 7.1, forms three microchannel pairs. Using these three pairs, three drugs spots (containing single drug or different drugs) can be created in a single gel layer. The membrane patterning consists of three pairs of open pore patches. These patches are arranged such as to align properly with the microchannels. 
Master's Thesis - Sarvesh Upadhyaya - McMaster University - Mechanical Engineering
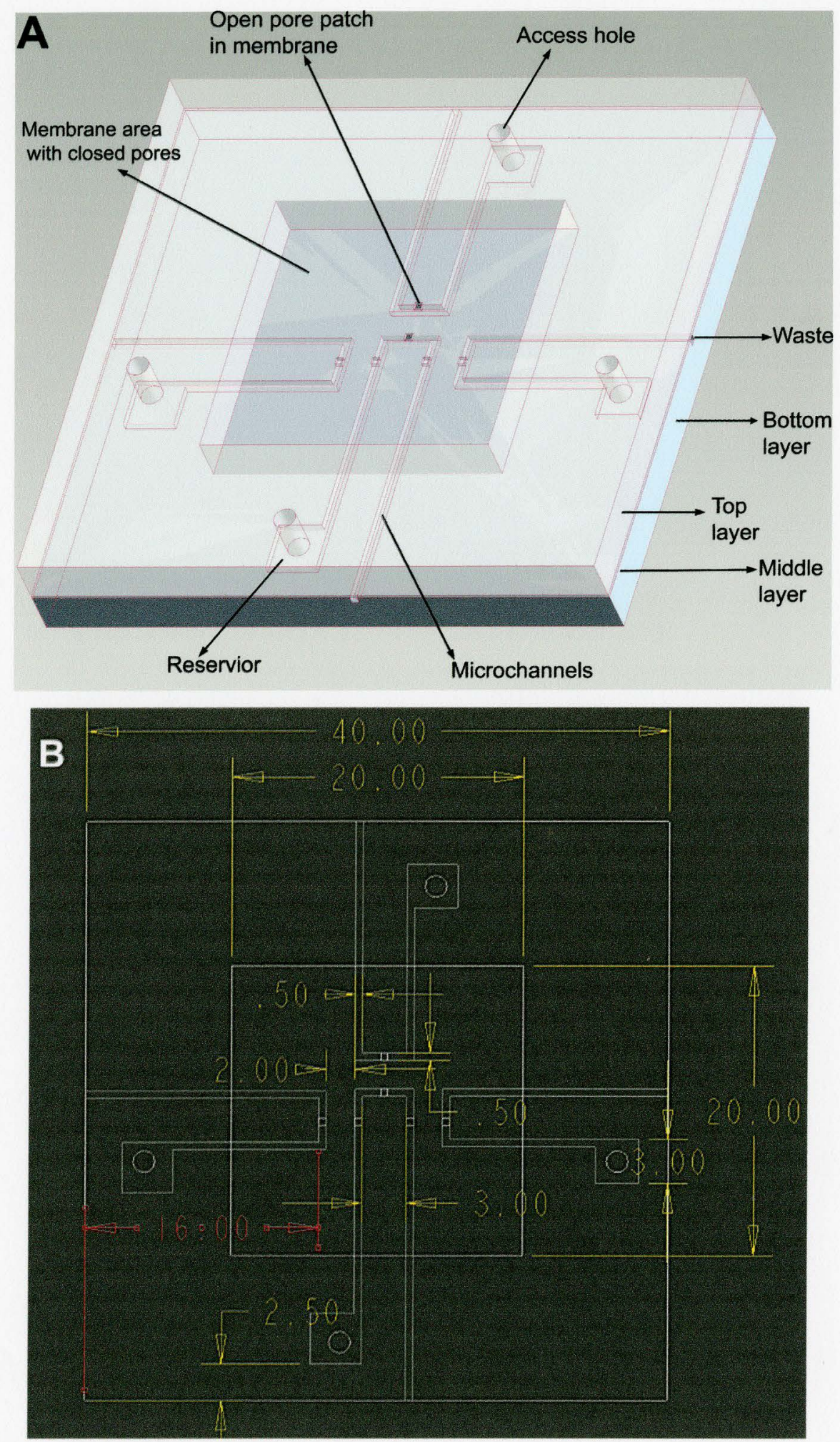

Figure 7.1 Three spot forming device design: (A): 3D schematic. (B). Typical dimensions. 
Master's Thesis - Sarvesh Upadhyaya - McMaster University - Mechanical Engineering

\subsubsection{Microarray Device}

The 3D schematic and typical dimensions of the device are shown in Figure 7.2A and 7.2B respectively. The bottom PDMS layer has open reservoir (in contrast to other devices having microchannels). Use of this reservoir is analogous to the use of microchannels in previous devices and it is filled with the drug to be pumped. The membrane is patterned is such a way to leave a microarray of open pore patches. Each such patch would produce a single spot. Two designs for microarray device having 44.4 spots $/ \mathrm{cm}^{2}$ (the density of patches on the membrane) and $156.25 \mathrm{spots} / \mathrm{cm}^{2}$ are made subsequently. In comparison with conventional well plate dimensions $(12 \mathrm{~cm} \times 8 \mathrm{~cm})$ these densities will lead to $\sim 4,000$ and 15,000 wells/plate which is an order of magnitude increase.

\subsection{DEVICE FABRICATION}

The fabrication process of three spot forming device is same as that of single spot forming device, described earlier (section 6.2). In this case a different photomask design is used to get a different microchannel network. To fabricate a microarray device, a different approach (as compared to previous devices) is used. Three thin sheets of PDMS $(3 \mathrm{~cm} \times 3 \mathrm{~cm} \times 4 \mathrm{~mm}, 3 \mathrm{~cm} \times 3 \mathrm{~cm} \times 3 \mathrm{~mm}, 3 \mathrm{~cm} \times 3 \mathrm{~cm} \times 1 \mathrm{~mm})$ are cut from large PDMS sheets. The large PDMS sheets in turn is produced by casting liquid PDMS in a petridish. Holes of $20 \mathrm{~mm} \times 20 \mathrm{~mm}$ are cut in two PDMS respectively. The third sheet is left without any hole in it. Membrane patterning method is same as described earlier (section 5.2.4). The stamp used is suitable to create microarray of open pore patches on the membrane. The fabrication process flow for microarray device is shown in Figure 7.3. 
Master's Thesis - Sarvesh Upadhyaya - McMaster University - Mechanical Engineering
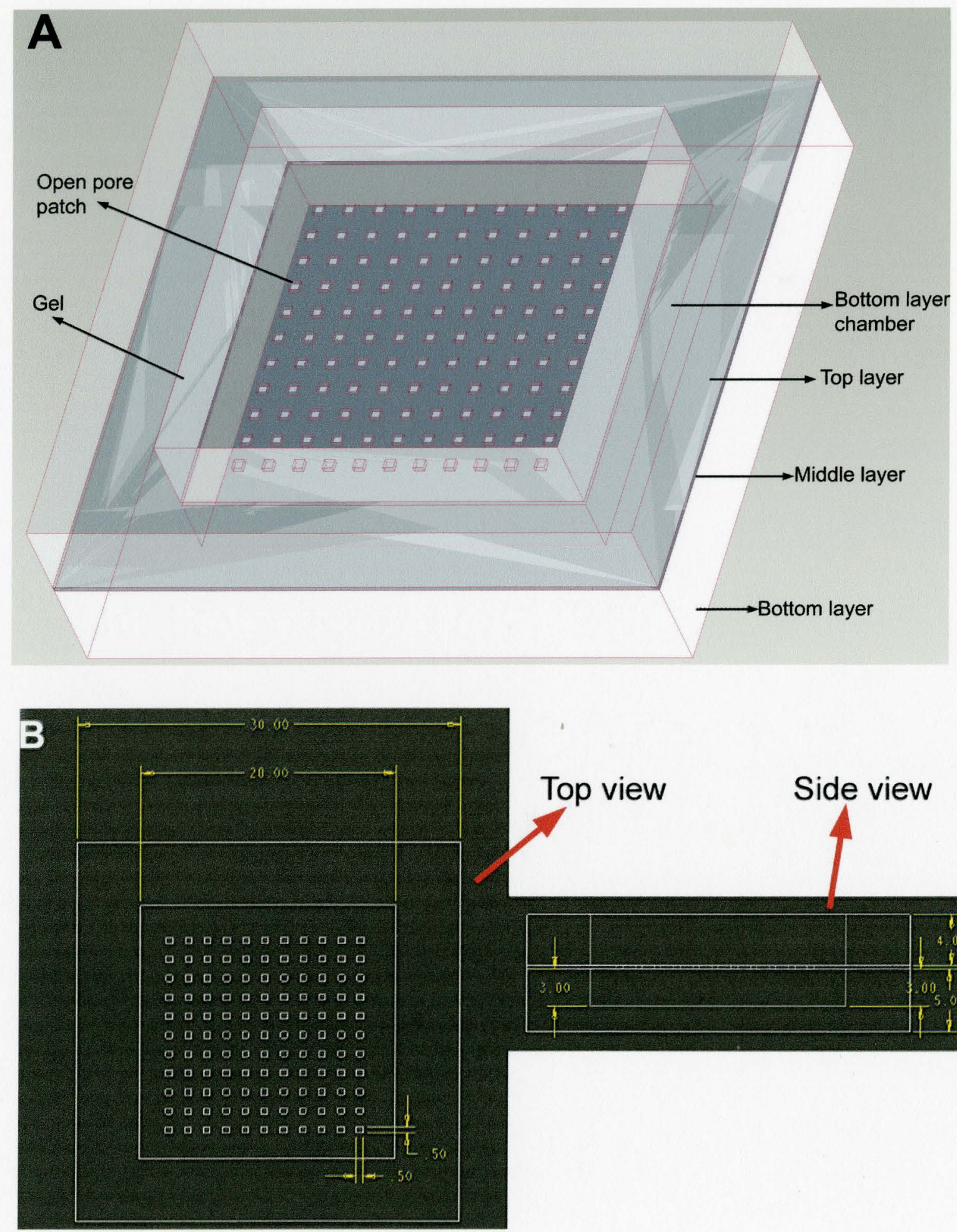

Figure 7.2 Microarray forming device design: (A): 3D schematic. (B) Typical Dimensions. 

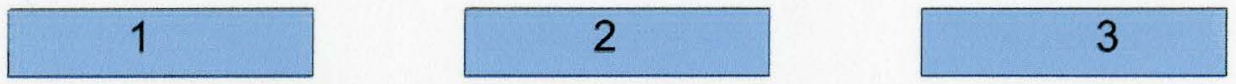

Three PDMS sheets were cut from PDMS sheets casted in petridish

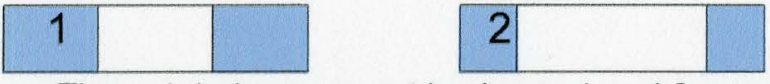

Through holes were cut in sheets 1 and 2 respectevily.

3rd sheet was left without any holes.

Membrane was patterned using the same method as described previously (heading 5.24 of this thesis).

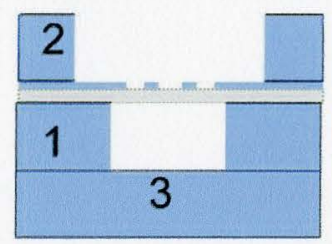

All three PDMS sheets and the membrane were stacked above each other as shown.

Figure 7.3 Fabrication process flow for microarray forming device design. The process flow for this device is different as there are no microchannels in the bottom PDMS layer. A reservoir serves in place of microchannels.

For electric field application, one electrode is inserted in to bottom PDMS reservoir. The second electrode is placed in a water layer filled on the top of agar gel. This avoids any damage to agar gel due to electrode insertion.

\subsection{Creation of independent drug spots}

$2 \%$ agar gel is used as the gel medium and trypan blue as testing compound. As shown in Figure 7.4, three pairs of microchannels are filled with trypan blue. When an electric potential is applied between two of the three pairs, the pumping of drug in two 
Master's Thesis - Sarvesh Upadhyaya - McMaster University - Mechanical Engineering

pairs start (only those experiencing electric potential). No drug is visually observed in the third spot. This demonstrates that the device has the capability of selectively switching on or off an individual spot out of a number of spots.
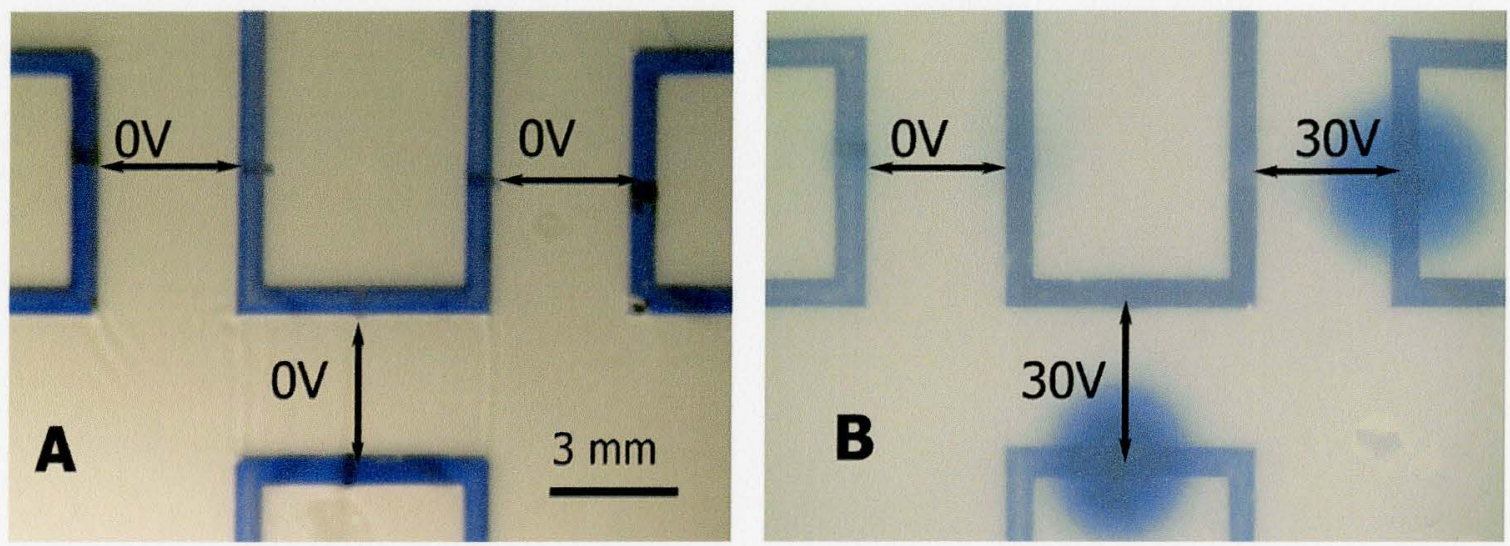

Figure 7.4 Independent spot creation. A: Top view of a device with three microspots and with no electromigration. B: Two out of three channels switched on to create two separate spots while there is no drug pumped at the third spot.

\subsection{Microarray Formation}

Microarray forming device is used in this experiment. A potential of $15 \mathrm{~V}$ was applied for 5 seconds. Figure 7.5 shows the microarray of trypan blue in agar gel. The created spot density is 44.4 spots $/ \mathrm{cm}^{2}$. Presence of regions with pumped trypan blue and regions with diffused trypan blue, as explained in section, can be observed.

\subsection{Microarray of Biomolecules}

Bovine Albumin Serum (BSA) and short length DNA are selected as model biomolecules in the protein and nucleic acid class. 
Master's Thesis - Sarvesh Upadhyaya - McMaster University - Mechanical Engineering

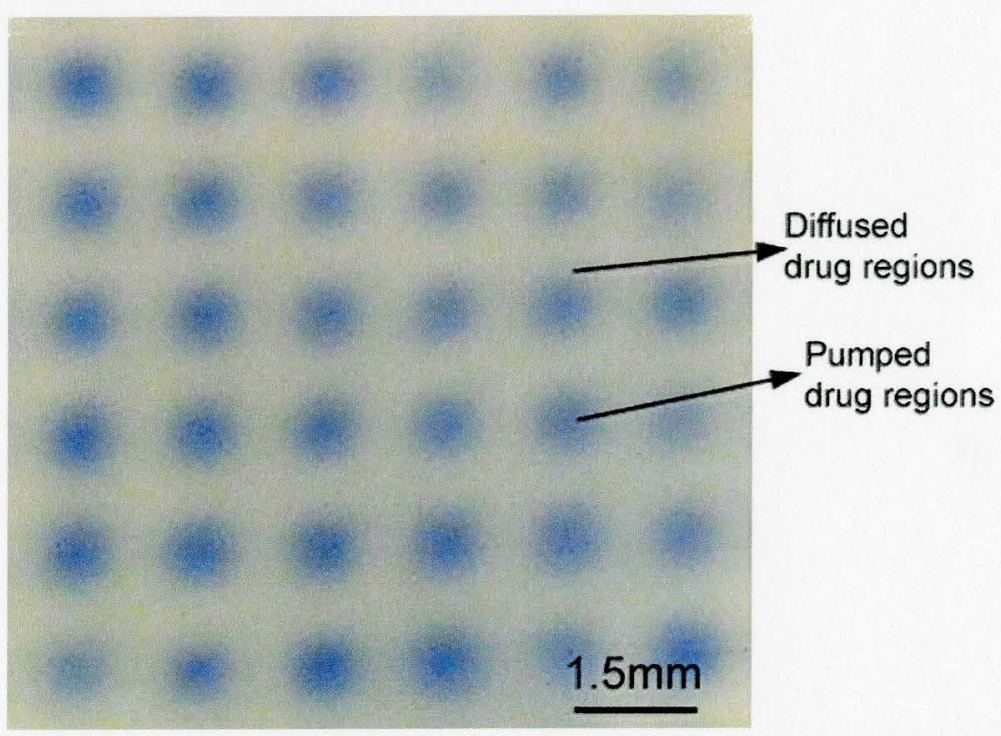

Figure 7.5 Microarray of drug spots: Trypan blue spots in agar gel. Each spot has two distinct regions, the pumped drug region (formed due to pumping through nanopores) and diffused drug region (formed due to diffusion in gel).

\subsubsection{Protein Microarrays}

A $10 \mathrm{mg} / \mathrm{ml}$ solution of BSA in phosphate buffer having $\mathrm{pH}$ of 7 was prepared to carry out the test. A potential of $10 \mathrm{~V}$ is applied for 20 seconds. After protein pumping, the gel (1\%) is separated from the device and stained using SafeBlue stain (details in appendix 4). This experiment is done with devices having different spot densities (44.4 spots $/ \mathrm{cm}^{2}$ and $156.25 \mathrm{spots} / \mathrm{cm}^{2}$ ). The created microarrays are shown in Figure 7.6. Blue regions are the areas with BSA bound with staining molecules. It can be observed that for higher spot densities (Figure 7.6B) the spots are rounder. This happens due to two reasons. First, the reflow of PDMS that happens during patterning process of membrane is more dominant for small size features. Secondly, some diffusion of protein takes place which is again more pronounced for smaller spot densities. 


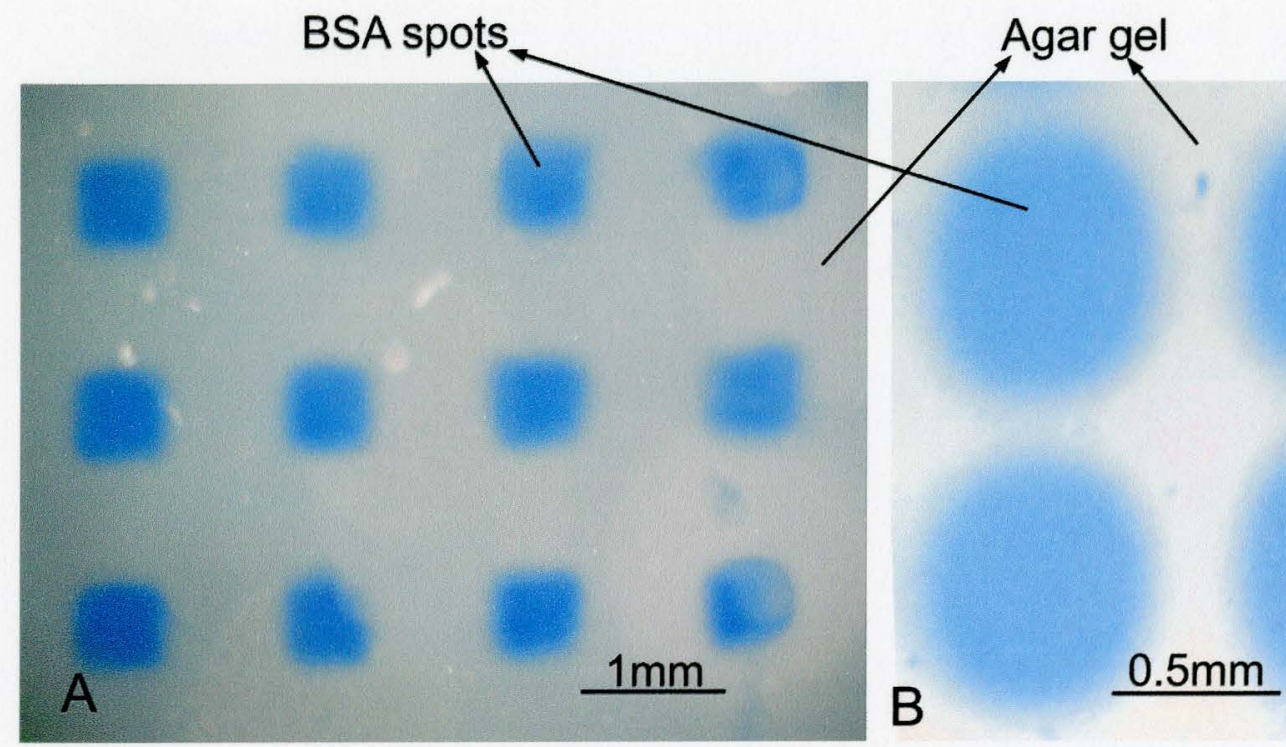

Figure 7.6 Microarray of protein spots. Microarray of protein (BSA stained blue). (A) $44.4 \mathrm{spots} / \mathrm{cm}^{2}$ (B) $156.25 \mathrm{spots} / \mathrm{cm}^{2}$.

\subsubsection{DNA Microarray}

In this experiment $40 \mu \mathrm{g} / \mathrm{ml}$ DNA (20bp long) solution in Tris, Acetate, and EDTA (TAE) buffer is used for this experiment. The solution concentration is tris$0.04 \mathrm{M}$, acetate- $0.04 \mathrm{M}$, EDTA-0.001M and solution $\mathrm{pH}$ of $8.3-8.5$. DNA is pumped through microarray forming device by applying $30 \mathrm{~V}$ for different duration of time $(5 \mathrm{sec}$, $10 \mathrm{sec}$ and $30 \mathrm{sec})$. Agar gel (3\%) used in this experiment is mixed with $0.5 \mu \mathrm{g} / \mathrm{ml}$ of ethidium bromide (EtBr). After the pumping the gel slab is washed using TAE buffer and distilled water. Then the gel slab is photographed by illuminating with UV light. The results are shown in Figure 7.7. For smaller time during pumping a microarray of DNA molecules (brighter regions, due to florescence of $\mathrm{EtBr}$ ) can be seen. For longer pumping time duration, mixing between adjacent spots happens (Figure 7.7C). 
Master's Thesis - Sarvesh Upadhyaya - McMaster University - Mechanical Engineering

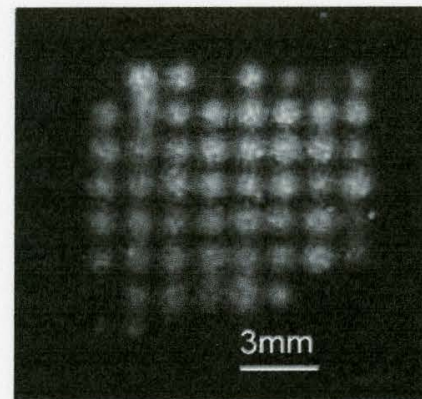

A

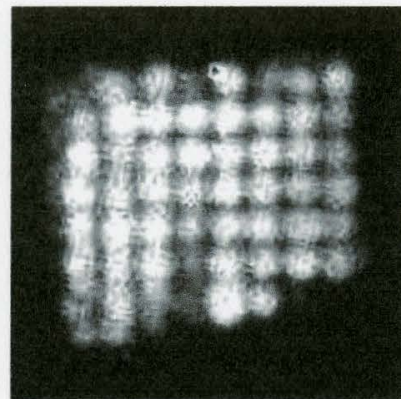

B

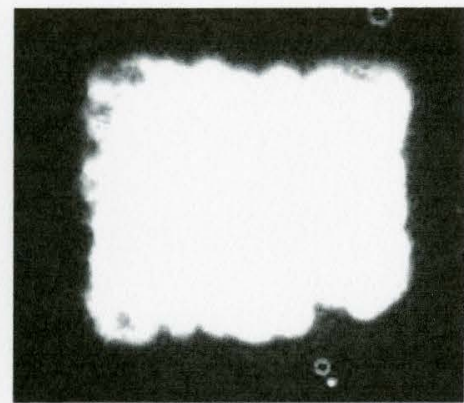

C

Figure 7.7 Microarray of DNA spots. Microarray of DNA (fluorescent with EtBr). Spot density 44.4 spots/ $\mathrm{cm}^{2}$. (A) Pumping duration 5sec, (B) pumping duration $10 \mathrm{sec}$, (C) Pumping duration $30 \mathrm{sec}$. A constant white background is seen due to saturated fluorescence of EtBr and mixing between spots.

\subsection{SPOT-SPOT INTERACTION DUE TO DIFFUSION}

The drug spot size grows as time increases. In case of a single spot device, it is not a problem because after some time, this diffusion is stopped by device walls. But in case of multiple spot devices, neighboring spots can touch each other.
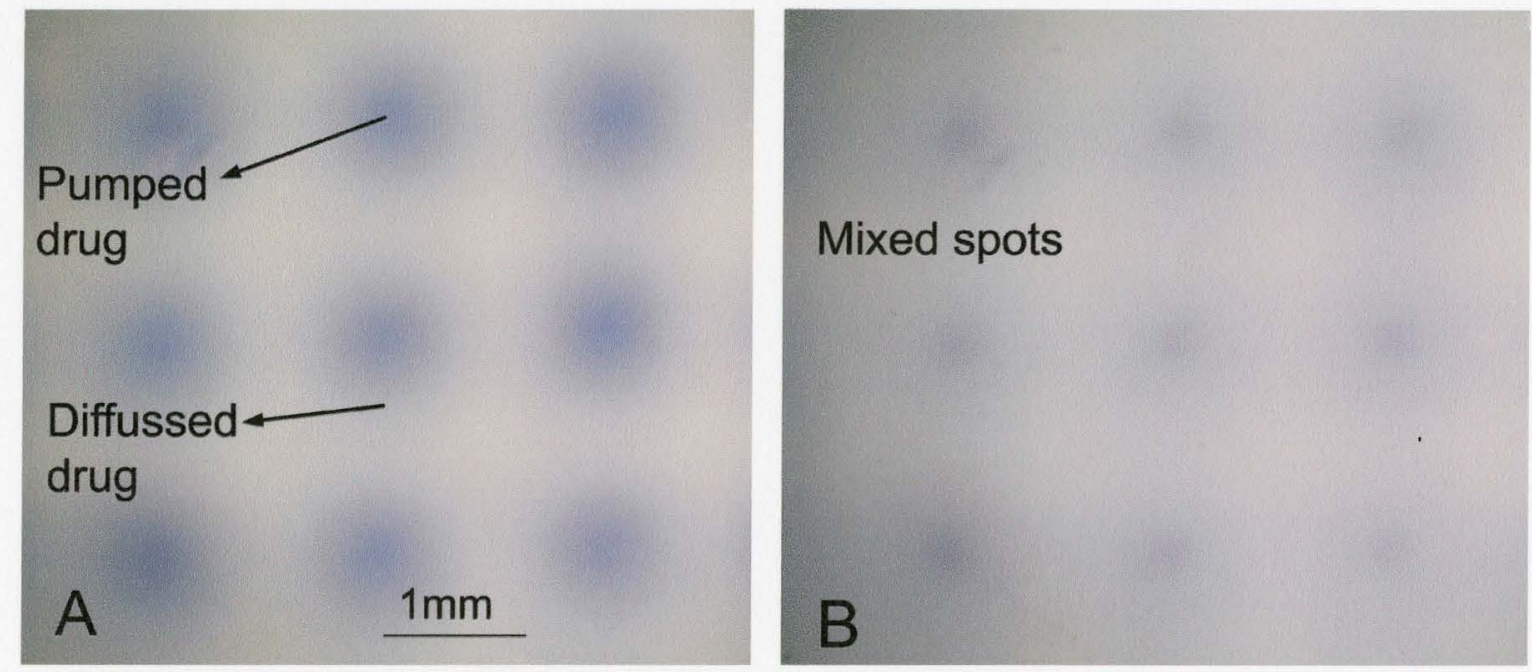

Figure 7.8 Mixing of drug spots. Microarray of trypan blue (A) one minutes after pumping, separate spots are visible (B) 15 minutes after pumping. Spots got mixed. 
Master's Thesis - Sarvesh Upadhyaya - McMaster University - Mechanical Engineering

This would lead to unsatisfactory performance of the device as it can cause mixing of adjacent spots. Figure 7.8 shows a microarray of trypan blue created by microarray device. Part A is 1 minute after the pumping with separate spots, while part B is after 15 minutes of pumping. In Figure 7.8B, due to diffusion of drugs, spots are not clearly separated (boundaries not clear) from one another. This mixing limits the higher spot densities (hence the number of experiments per device) that can be achieved.

With the help of three spot forming device and microarray forming device, creation of independent drug spot, microarray formation and pumping of biomolecules have been demonstrated. It was observed that diffusion is a limitation on spot densities that can be achieved. The next chapter deals with numerical analysis drug diffusion in gel to find out methods for diffusion reduction (optimization). 
Master's Thesis - Sarvesh Upadhyaya - McMaster University - Mechanical Engineering

\section{CHAPTER 8}

\section{MOLECULAR DIFFUSSION BARRIER}

\section{FOR GEL SLAB}

As described in previous chapter, for lower molecular weight molecules (having large diffusional constants) the diffusion in gel slab caused interspot mixing for too closely spaced spots. In order to find a method to decrease the distance between adjacent spots (i.e. to increase the spot density) a numerical study of diffusion in different agar gel geometries is done. The numerical simulation is done using COMSOL Multiphysics. Based on this study a diffusion barrier (thinner regions in gel slab having higher resistance to diffusion) is proposed that would help in reducing the spreading of drugs in drug spots.

\subsection{The physics of the problem}

Agar gel is made of 3D network of fibers that consists of large size $(\sim 10-100 \mathrm{~nm})$ pores [Lahaye 1991]. Due to presence of fibrous network there is no convective flow present in agar gel slab [Gutenwik 2004]. But molecules having radius smaller than the gel pores can diffuse through these pores. The pore sizes of agar gel for different agar concentrations are shown in Figure 8.1 [Lebrun 1993, Ackers 1962]. The pore sizes decrease with increasing gel concentrations, but for typical gel concentrations (1-3\%) 
Master's Thesis - Sarvesh Upadhyaya - McMaster University - Mechanical Engineering

used in present study the pores sizes $(35-90 \mathrm{~nm})$ are much larger than the molecules used. For example the molecular size of BSA is $14 \mathrm{~nm}$ (taken from supplier data sheet, details in appendix 5). BSA is a large protein molecule (M.W. 66,430) used in present study. Hence it can be assumed that for most of the molecules, molecular diffusion would take place.

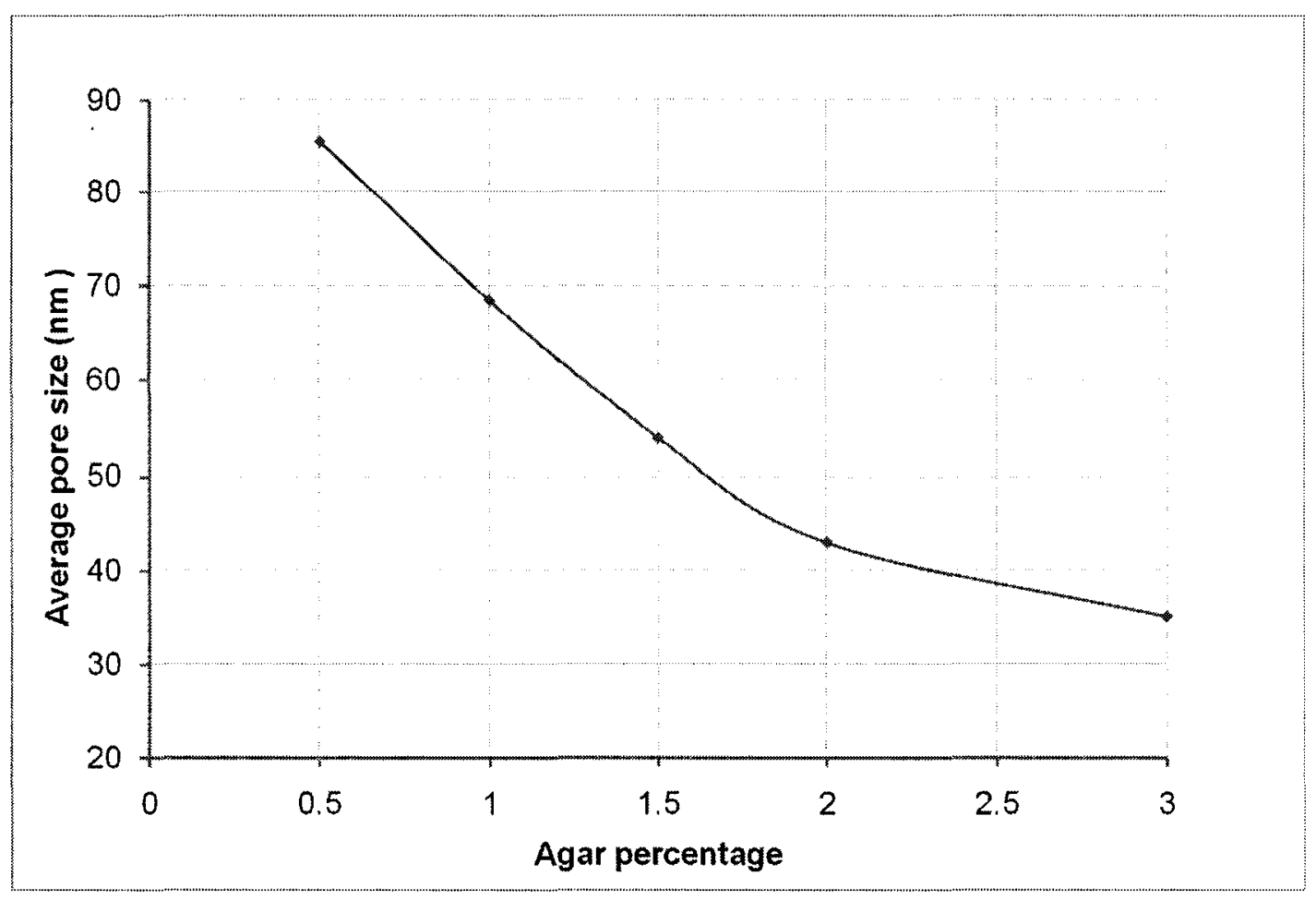

Figure 8.1 Average pore size vs. gel concentration for Agar gel. [Lebrun 1993, Ackers 1962].

The diffusion behavior in gel can be taken to be molecular diffusion in single phase governed by Fick's law of molecular diffusion [Gutenwik 2004]. Fick's second law, for constant diffusivity and no generation term, is given by following equation [Welty 2000]. 
Master's Thesis - Sarvesh Upadhyaya - McMaster University - Mechanical Engineering

$$
\frac{\partial c}{\partial t}=D_{e}\left(\nabla^{2} c\right)
$$

Where $D_{e}$ is the effective diffusion constant and $c$ is concentration. Diffusion of drugs in gel will be modeled based on above equation.

\subsection{Diffusional Mass Transport in Gel Slabs having Different}

\section{Thicknesses}

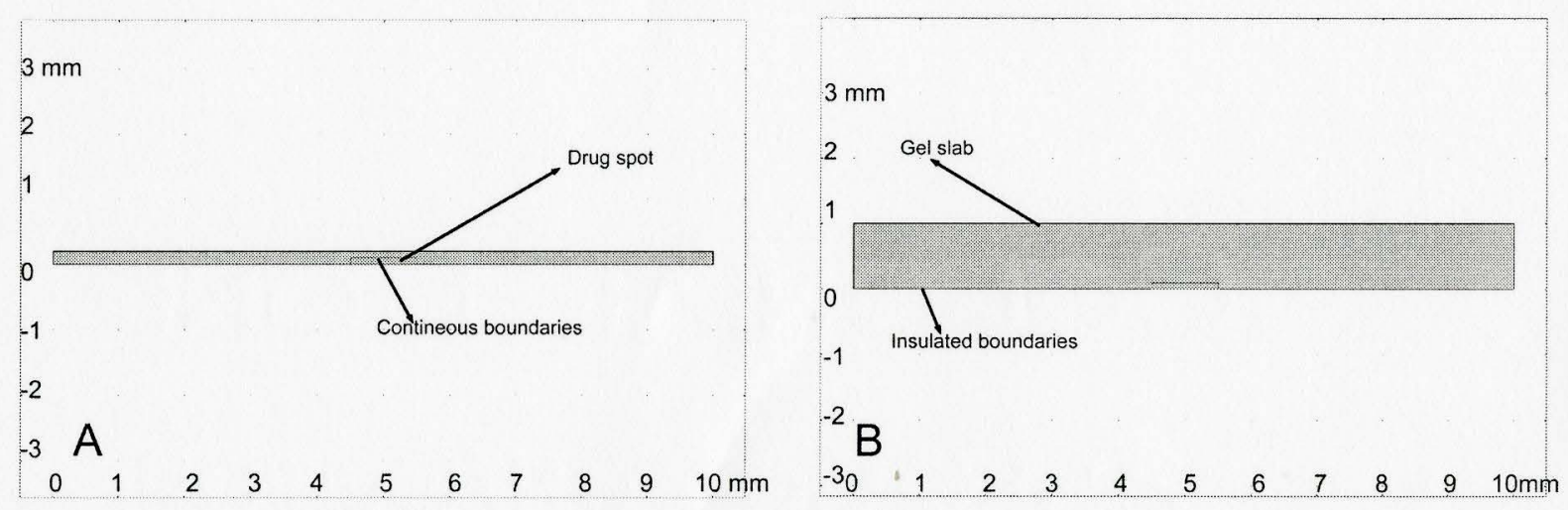

Figure 8.2 Gel slabs for modeling. Two gel slabs having $0.2 \mathrm{~mm}$ and $1 \mathrm{~mm}$ thickness are modeled. Other dimensions like width of gel and drug spot size are identical.

The effective mass transport occuring due to molecular diffusion is the parameter responsible for inter-spot mixing. It is modeled in gel slabs having different thickness to find of the effect of thickness. All parameters except gel slab thickness (gel concentration, width, and diffusion constants) are kept identical. 
Master's Thesis - Sarvesh Upadhyaya - McMaster University - Mechanical Engineering

\subsubsection{The Problem Definition}

In present case the drug diffusion in vertical direction (i.e. gel thickness, y direction) and horizontal direction (x) are studied. For computational economy the gel slabs are modeled in 2D, by considering $\mathrm{x}$ and $\mathrm{y}$ direction, $\mathrm{z}$ direction is assumed unity in length. Different gel slab dimensions $(10 \mathrm{~mm} \times 0.2 \mathrm{~mm}$ and $10 \times 1 \mathrm{~mm})$ are used to study the effect of slab dimension on the diffusion. Although the actual shape of drug spot is complex, for simplicity, an $1 \mathrm{~mm} \times 0.1 \mathrm{~mm}$ size region is assumed to be the initial drug spot (i.e. spot just after pumping). It is assumed that initially such an amount of BSA is present in the drug spot that after complete ideal diffusion (same concentration everywhere) the BSA concentration in gel will be $10^{-4} \mathrm{M}$. This assumption is made in order to be able to compare diffusion behavior in gel slabs having different volumes. More ever, as compared to initial drug spot concentration, concentrations after diffusion are more important for the intended applications of the device. All the boundaries of the gel slab are treated to have Neumann boundary condition (i.e. no diffusion can take place through them).

\subsubsection{Numerical Simulation in COMSOL Multiphysics}

Chemical engineering 2D transient analysis module of Comsol Multiphysics 3.3 is used for this simulation. Two slabs as shown in Figure 8.2 are modeled. SI units are used for calculations. The gel dimension in $\mathrm{x}, \mathrm{y}$ and $\mathrm{z}$ are $10 \mathrm{~mm}, 0.2 \mathrm{~mm}$ (or $1 \mathrm{~mm}$ ) and unity $(1 \mathrm{~m})$ respectively. Each slab has smaller $1 \mathrm{~mm} \times 0.1 \mathrm{~mm}$ subdomains in their bottom middle part (as shown in Figure 8.2). These subdomains are assumed to be the initial drug spots (just after pumping is over). 
Master's Thesis - Sarvesh Upadhyaya - McMaster University - Mechanical Engineering

The diffusion constant of BSA is taken to be $3 \times 10^{-11} \mathrm{~m}^{2} / \mathrm{s}$ for $2 \%$ agar gel at room temperature [Ackers 1962]. Every boundary is assumed to have Neumann boundary condition. 22030 and 40784 elements were generated after meshing of thinner and thicker gels respectively. A finer mesh is used in drug spot regions to capture diffusion accurately. Further refining of the mess did not result in significant changes. Transient analysis is used for $0-20000$ seconds.

\subsubsection{Comparison of Concentration Distribution}

Figure $8.3 \mathrm{a}$ and Figure $8.3 \mathrm{~b}$ show the concentration distributions at time $\mathrm{t}=20000$ sec for thinner and thicker gels respectively. As expected (due to identical the diffusion properties and the lateral dimensions) a very similar concentration distribution is observed. Figure 8.4 shows the plot of concentration vs. $\mathrm{x}$ axis position at $\mathrm{t}=20000$ for thinner and thicker slabs respectively. Again, it can be observed that concentration profiles are almost identical in both cases.

\subsubsection{Comparison of Mass Transport}

To compare the mass transport effects, the diffusional flux (mass transport per unit time per unit cross sectional area) crossing a line $\mathrm{x}=\mathrm{x}_{1}=0.008$, between time $\mathrm{t}=\mathrm{t}_{1}=0$ to $t=t_{2}=20000$ can be computed by two methods. Either by integrating mass flux that cross this line between $t_{1}=0$ to $t_{2}=20000$, or by integration concentration over the region beyond this line (ie. rectangle diametrically bound by points $\mathrm{x}_{1}=0.008, \mathrm{y}_{1}=0$ and $\left.\mathrm{x}_{2}=0.01, \mathrm{y}_{2}=0.001\right)$. Mathematically: 
Master's Thesis - Sarvesh Upadhyaya - McMaster University - Mechanical Engineering
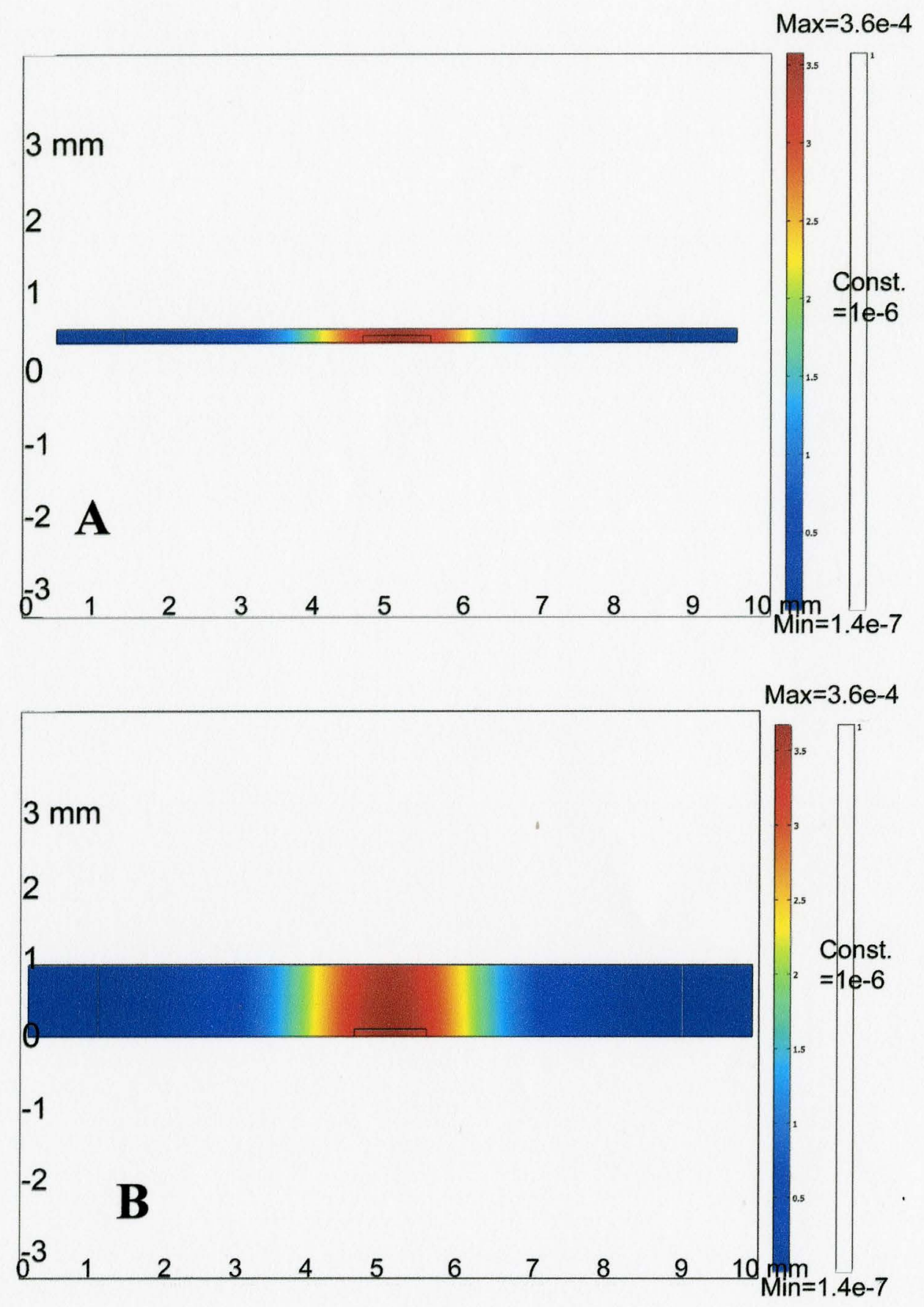

Figure 8.3 Comparison of concentration distribution in two gel slabs having identical drug spot: The distribution is almost identical, $10^{-6} \mathrm{M}$ concentration (shown by white line in colored images) occur at $\sim 4.2 \mathrm{~mm}$ from the center of drug spots. 
Master's Thesis - Sarvesh Upadhyaya - McMaster University - Mechanical Engineering

MassTrasport $=\left(\int_{t_{1}}^{t_{2}} \int_{y_{1}}^{y_{2}}(D f l u x) d y d t\right)_{x=x_{1}}=\left(\int_{x_{1}}^{x_{2}} \int_{y_{1}}^{y_{2}}(c) d y d x\right)_{t_{2}}$

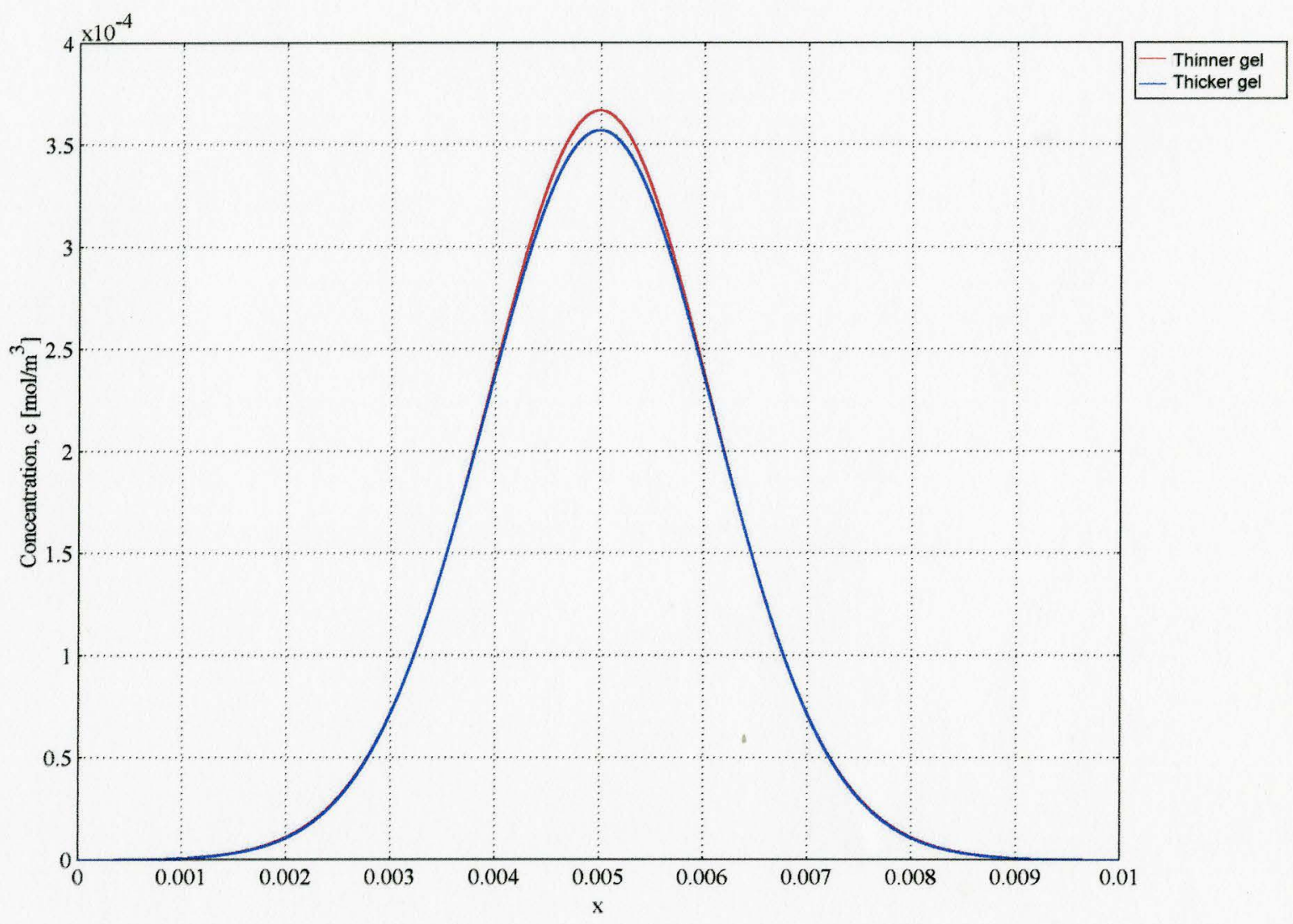

Figure 8.4 Concentration profile comparison of thin and thick gel: Concentrations at bottom of gel (i.e. $y=0$ ) at time $t=20000$ sec. Both the gels have almost identical profiles.

Where 'Dflux' is diffusion flux at any time and point on the line $x^{=} x_{1}$ and ' $c$ ' is concentration. The total amount of drug that has crossed the line $\mathrm{x}=0.8$ is calculated for both the gels. For thinner gel it is $9.3 \times 10^{-13}$ moles, and for thicker gel, it is $5.19 \times 10^{-12}$ moles. The ratio of these amounts is roughly $\sim 5$ (i.e. the ratio between gel slab thickness). 
Master's Thesis - Sarvesh Upadhyaya - McMaster University - Mechanical Engineering

This approximate relation between mass transport and gel thickness is expected as concentration profiles are very similar.

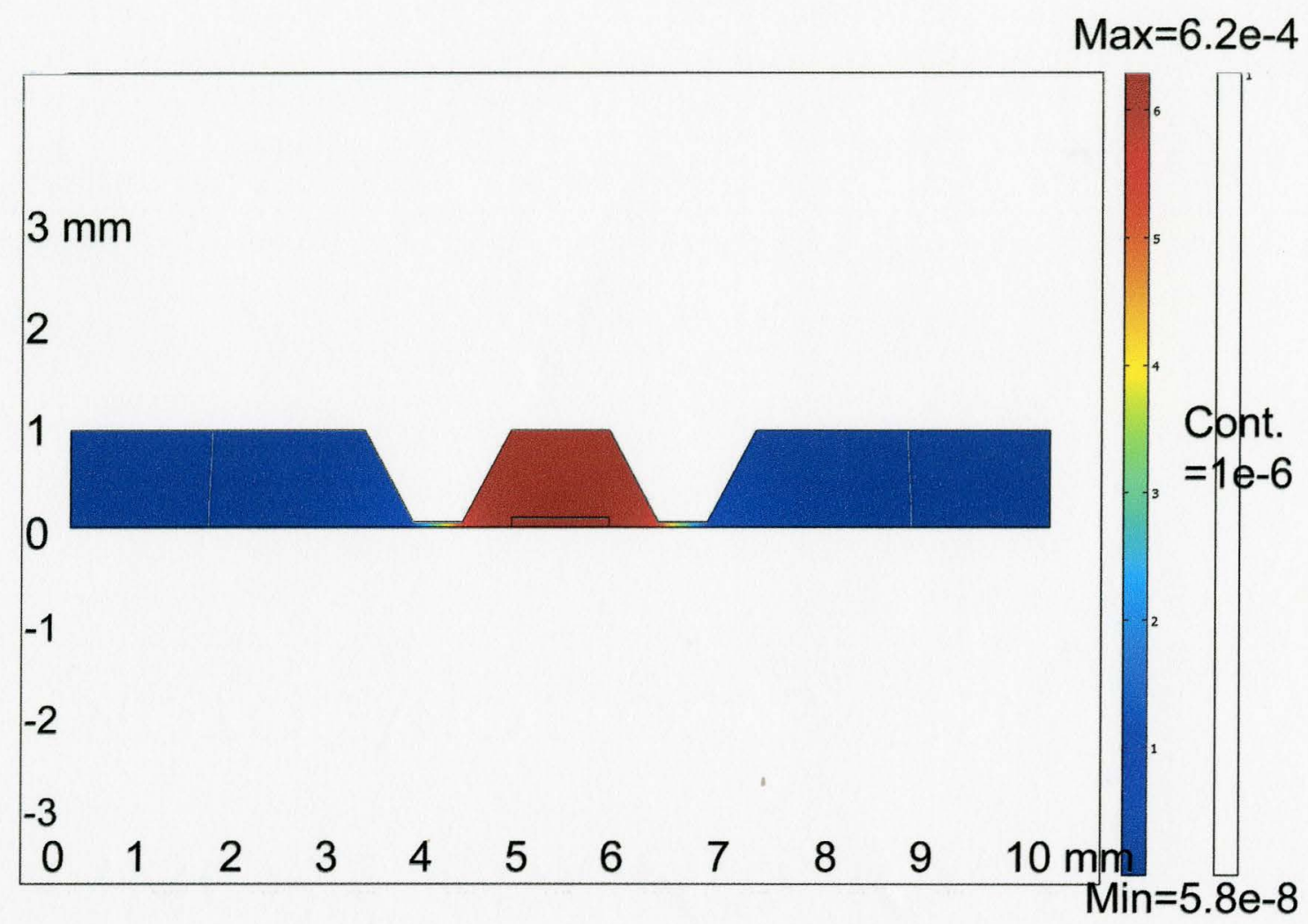

Figure 8.5 Concentration in $1 \mathrm{~mm}$ thick gel slab with diffusion barrier. On comparing with $1 \mathrm{~mm}$ thick gel without barrier (figure 8.3b) the drug is concentrated between the barriers (the thinner regions), and 10-6M concentration line (shown by white line is) is at $\sim 3.7 \mathrm{~mm}$ from the center of drug spot.

Based on this numerical analysis it is concluded that for current setup, the mass transport due to diffusion is approximately proportional to the thickness of the gel. This is used for designing of diffusion barriers in gel slab as described in next section. 
Master's Thesis - Sarvesh Upadhyaya - McMaster University - Mechanical Engineering

\subsection{Diffusion Barrier in Gel Slab}

The net mass transport is dependent upon gel thickness, hence in order to reduce the mass transport between adjacent spots, thinner gel regions are introduced alternatively between spots. By this arrangement each spot would be surrounded by four such barriers from all four sides. To estimate the effects of such barrier on diffusional mass transport a typical example case is numerically studied. 2D numerical transient analysis is done for time $0-20000$ seconds. The number of elements used is 50782 . Figure 8.5 shows the concentration distribution of the gel slab with diffusion barrier. This concentration distribution is compared with a simple $1 \mathrm{~mm}$ thick gel having same dimensions (described in previous section).

\subsubsection{Comparison with simple gel Slab}

On comparing the concentration distribution of gel slabs with and without diffusion barrier (Figure 8.5 and $8.3 \mathrm{~b}$ ) it can be noticed that for former case most of the high concentration area is between the diffusion barriers, while in second case it is smoothly spread. It should be noted that initial concentrations in drug spots for both the gels are such that, if the drug is uniformly spread in the gels, it will result in identical concentrations.

Figure 8.6 shows comparison of concentration distribution for line $y=0$, for $0<\mathrm{x}<0.01$ at time $\mathrm{t}=2000$ for both the cases (i.e. gel slab with barrier, and without barrier). 
Master's Thesis - Sarvesh Upadhyaya - McMaster University - Mechanical Engineering

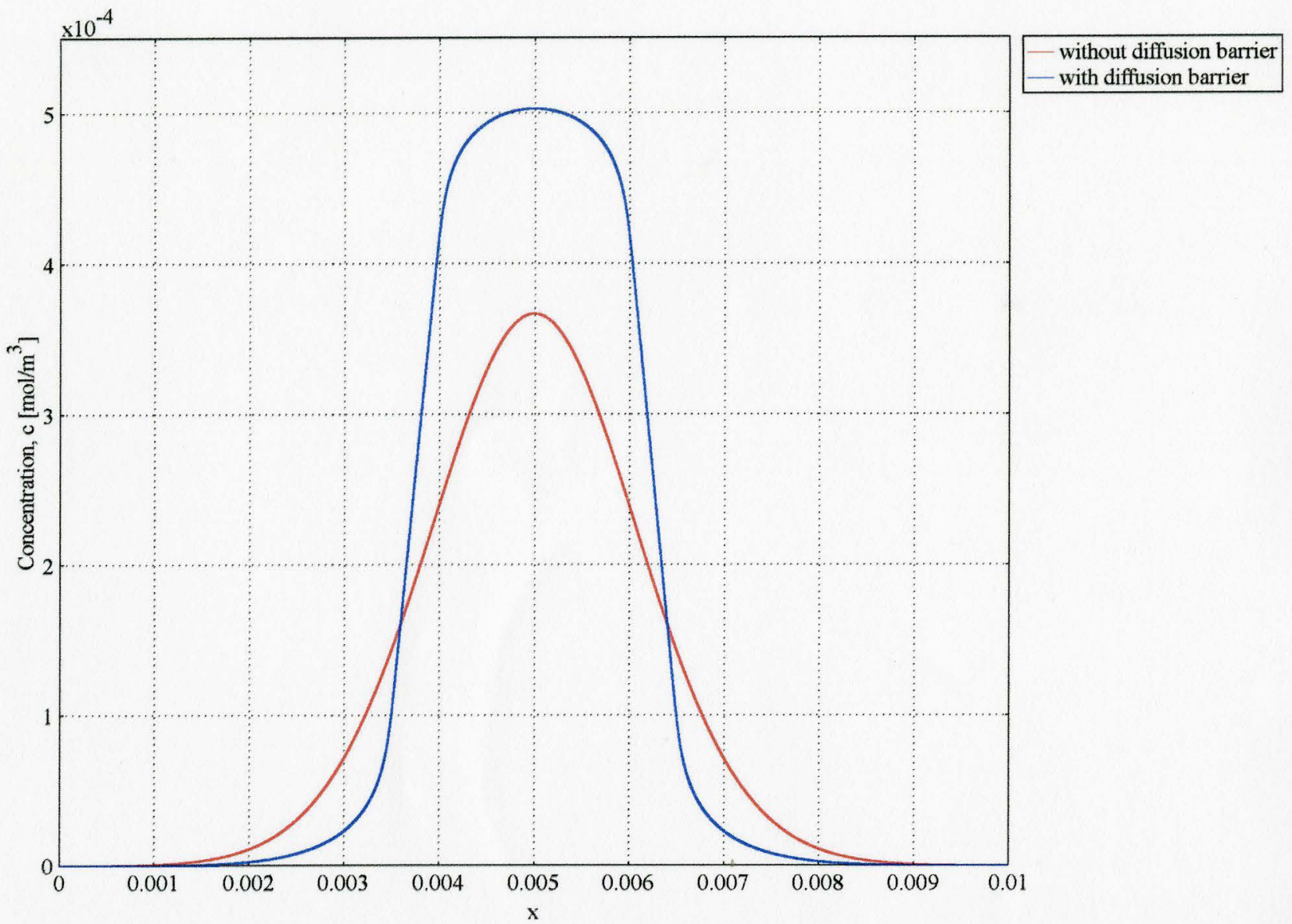

Figure 8.6 Concentration profile comparison of gel with and without diffusion barrier: Concentrations at bottom of gel (i.e. $y=0$ ) at time $t=20000 \mathrm{sec}$. Gel with barrier have high concentration of drug in the spot while most of concentration falls in barriers.

It can be noticed that for gel slab with barrier the concentration distribution between regions $.045<\mathrm{x}<.055$ is flatter as compared to that of without barrier. Concentration gradients are high in between $.055<\mathrm{x}<.065$ (the diffusion barrier) and concentrations in this zone falls rapidly. Similarly Figure 8.7 shows the concentration distribution for line $\mathrm{x}=0.005$ and $0<\mathrm{y}<0.001$ for both the cases. Here too it can be seen 
Master's Thesis - Sarvesh Upadhyaya - McMaster University - Mechanical Engineering

that, concentrations are higher and flatter in case of diffusion barrier (i.e. drug is retained in the spot and more uniformly distributed).

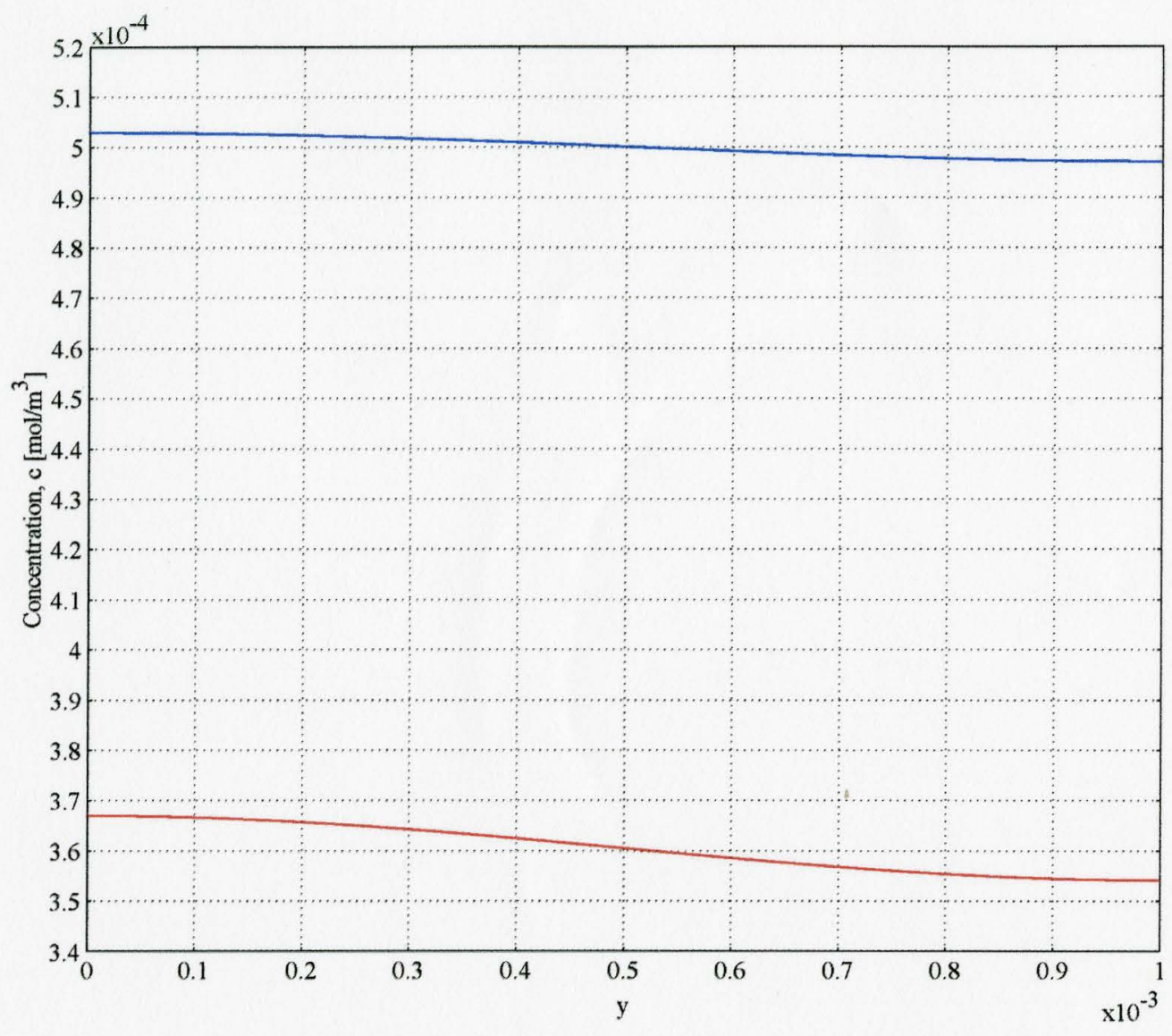

Figure 8.7 Concentration profile comparison of gel with and without diffusion barrier: Concentrations at middle of drug spot (i.e. $x=0.005 \mathrm{~m})$ and $(0<y<.001 \mathrm{~m})$ at time $t$ $=20000 \mathrm{sec}$. Gel with barrier retains the drug in the spot and have more uniform drug distribution (i.e. blue curve is flatter).

By above analysis it is concluded that, diffusion barrier limits the spread of drug in agar gel slab. It also leads towards more uniformly distribution of drug in a single spot. 
Master's Thesis - Sarvesh Upadhyaya - McMaster University - Mechanical Engineering

This can be used for development of a microarray device with higher spot density with lesser chance of interspot mixing. 
Master's Thesis - Sarvesh Upadhyaya - McMaster University - Mechanical Engineering

\section{CHAPTER 9}

\section{CONTRIBUTIONS AND FUTURE WORK}

\subsection{CONTRIBUTIONS}

The objective of the current work was to identify a suitable method to supply drugs in a microarray format for cell culture in a gel medium. Cell based HTS devices based on this method were to be designed, fabricated and tested.

During the course of this work following contributions to the field of microfluidics based HTS have been made.

\subsubsection{A Novel Idea for Cell Based HTS}

In contrast to existing microfluidic HTS devices, a multi-layered noncompartmentalized, cell culture approach has been developed. The bottom layer acted as support structure consisting of a network of microfluidic channels. The middle layer, which is a very thin porous membrane, acted as a group of pumps. The top layer is made from agar gel (a traditional culture material). The main advantages from this approach are that the culture surface area is analogous to cell culture agar plates. This approach is without confinement on cell growth and proliferation that may occur due to compartmentalization of culture area. As drug dosing is done with the help of nanopores, 
Master's Thesis - Sarvesh Upadhyaya - McMaster University - Mechanical Engineering

theoretically, by using a single nanopore this approach also have the ultra precise dosing potential.

\subsubsection{Design Fabrication and Testing of Microfluidic HTS Devices}

A number of devices (single drug spot forming devices, 3 spot forming devices, microarray forming devices) based on above idea, were designed and fabricated. The fabrication process also involved PDMS microcontact printing of nanoporous membranes. The devices were tested on a number of compounds representing typical intended molecules that will be used in the devices (model drug molecules, protein, and DNA).

Drug spot sizes ranging from $200 \mu \mathrm{m}$ to $6 \mathrm{~mm}$ diameter have been created. Drug dose variation with time was studied and doses between 50-3000 $\mu \mathrm{g}$ of model drug (trypan blue) have been successfully pumped into the gel. Drug spot growth rate, both during pumping and post pumping was studied. Model drugs as well as biomolecules (protein \&DNA) were successfully pumped in microarray format and visualized. Microarray density of 156.25 spots $/ \mathrm{cm}^{2}$ has been achieved. At well plate dimensions this density would give 15,000 spots/ plate.

\subsubsection{Diffusion Barrier}

Diffusion of molecules in agar gel was found to be a limiting factor on drug spot density. Numerical study of molecular diffusion in different gel slabs was done. Based on this study a method (i.e. diffusion barrier) for spot density maximization was proposed. The method was based on horizontal diffusion control (without altering 
Master's Thesis - Sarvesh Upadhyaya - McMaster University - Mechanical Engineering

diffusional constants) of drugs so that the spots can be placed closer to one another. The horizontal diffusion was controlled by putting diffusion barriers (thinner gel regions) in the gel slab separating spots. These thin regions provide high resistance to molecule diffusion hence reducing effective mass migration of molecules due to diffusion.

\subsection{FUTURE WORK}

Further investigation is needed in order to fully demonstrate and characterize the device capabilities. The following are recommended for future research:

- To show the device capability of supplying drugs to a lawn of cells grown in the device. Also to show the device capability of HTS format cell experimentation.

- Device characterization, which should include voltage vs. dose, and time vs. dose for varying molecular charges and molecular sizes. This work would help to predict the pumping characteristics of those compounds which have not been previously characterize for nanoporous membrane pumping.

- Fabrication and testing of a device based on diffusion barrier proposed. This work would help in achieving higher drug spot densities (i.e. higher throughput rates). 
Master's Thesis - Sarvesh Upadhyaya - McMaster University - Mechanical Engineering

\section{BIBLIOGRAPHY}

[Ackers 1962] G. K. Ackers and R. L. Steere "Restricted diffusion of macromolecules through agar-gel membranes", Biochimica Et Biophysica Acta, Vol.-59 pp.-137-149, 1962.

[Andersson 2003] H. Andersson, A. van den Berg, "Microfluidic devices for cellomics: a review", Sensors and Actuators B, Vol.-92, pp. 315-325, 2003.

[Balagadde 2005] F.K. Balagadde, L. C. You, C. L. Hansen, F. H. Arnold and S. R. Quake, "Long-term monitoring of bacteria undergoing programmed population control in a microchemostat", Science, Vol.-309, pp.137-140, 2005.

[Bassous 1977] E. Bassous, H.H. Hub and L. Kuhn, "Ink jet printing nozzle arrays etched in silicon", Applied Physics Letters, Vol.-31, pp. 135-137,1977.

[Bean 1972] C. P. Bean, "The physics of porous membranes" In: "Membranes". G. Eisenman, editor. pp 1-54. Marcel Dekker, New York, 1972.

[Beebe 2000] D. Beebe, "Microfabricated fluidic devices for single cell handling and analysis", in "Emerging Tools for Single Cell Analysis: Advances in Optical 
Master's Thesis - Sarvesh Upadhyaya - McMaster University - Mechanical Engineering

Measurement Technologies", ed. G Durack, J Robinson, Wiley Publication, New York, 2000.

[Bohrer 1984] M. P. Bohrer, G. D. Patterson, and P. J. Carroll, "Hindered diffusion of dextran and ficoll in microporous membranes", Macromolecules, Vol.-17, pp.1170-1173, 1984.

[Booth 2004] B. Booth and R. Zemmel "Prospects for productivity", Nature Reviews Drug Discovery, Vol.-3, pp.451-456, 2004.

[Bousse 1996] L. Bousse, "Whole cell biosensors", Sens. Actuators B, Vol.-34 pp. 270$275,1996$.

[Chen 1997] C. S. Chen, M. Mrksich, S. Huang, G. M. Whitesides, D. E. Ingber, "Geometric control of cell life and death", Science, Vol.-276, pp. 1425-1428, 1997.

[Chen 2002] C. H. Chen, J. S. Santiago, "A planar electroosmotic micropump", Journal of Micro Electro Mechanical Systems, Vol.-11, pp. 672-683, 2002.

[Christopoulos 1999] G. Christopoulos, K. J. Perry, M. Morfis, N. Tilakaratne, Y. Gao, N. J. Fraser, M. J. Main, S. M. Foord, and P. M. Sexton, "Multiple amylin receptors arise from receptor activity-modifying protein interaction with the calcitonin receptor gene product", Molecular Pharmacology, Vol.-56, pp. 235-242, 1999. 
Master's Thesis - Sarvesh Upadhyaya - McMaster University - Mechanical Engineering

[Croston 2002] G. E. Croston, "Functional cell-based uHTS in chemical genomic drug discovery", Trends in Biotechnology, Vol.-20, pp.110-115, 2002.

[Delamarche 1997] E. Delamarche, A. Bernard, H. Schmid, B. Michel, H. Biebuyck, "Patterned delivery of immunoglobulins to surfaces using microfluidic networks", Science Vol.-276, pp.779-781, 1997.

[Despont 1997] M. Despont H. Lorenz, N. Fahrni, J. Brugger, P. Renaud, P. Vettiger, "High-aspect-ratio, ultrathick, negative-tone near-UV photoresist for MEMS applications" 10th IEEE Micro Electro Mechanical Systems Conference, pp. 518, 1997.

[Dickson 2004] M. Dickson and J. P. Gagnon, "Key factors in the rising cost of new drug discovery and development", Drug Discovery Nature Review, Vol.-3, pp. 417-429, 2004.

[DiMasi 1991] J. A. DiMasi, R. W. Hansen, H. G. Grabowski, L. Lasagna, "Cost of innovation in the pharmaceutical industry", Journal of Health Economics, Vol.-10, pp.107-142, 1991.

[DiMasi 2003] J. A. DiMasi, R. W. Hansen, H. G. Grabowski, "The price of innovation: new estimates of drug development costs", Journal of Health Economics, Vol.-22, pp.151-185, 2003.

[Dittrich 2005] P. S. Dittrich and A. Manz "Lab-on-a-chip: Microfluidics in drug discovery", Drug Discovery Nature Review, Vol.-5, pp. 210-218, 2005. 
Master's Thesis - Sarvesh Upadhyaya - McMaster University - Mechanical Engineering

[Drews 2000] J. Drews, "Drug discovery: a historical perspective", Science, Vol.-287, pp.1960-1964, 2000.

[Effenhauser 1997] C.S. Effenhauser, G.J.M. Bruin, A. Paulus, "Integrated chip-based capillary electrophoresis", Electrophoresis Vo.-18 , pp.-2203-2213, 1997.

[Ellis 1991] R. E. Ellis,. J. Yuan, and H. R. Horvitz, "Mechanisms and functions of cell death", Annual Review in Cell Biology, Vol.-7, pp.663-698, 1991.

[Ertel 1994] S. I. Ertel, B. D. Ratner, A. Kaul, M. B. Schway, T. A. Horbett, "In vitro study of the intrinsic toxicity of synthetic surfaces to cells", Journal of Biomedical Materials Research, Vol.-28, pp.667-675, 1994.

[Feng 2003] R. Feng and R. J Farris, "Influence of processing conditions on the thermal and mechanical properties of SU8 negative photoresist coatings", Journal of Micromechanics and Microengineering, Vol.-13, pp. 80-88, 2003.

[Fielder 1998] S. Fiedler, S. Shirley, T. Schnelle, G. Fuhr, "Dielectrophoretic sorting of particles and cells in a microsystem", Analytical Chemistry, Vol.-70, pp. 1909-1915, 1998.

[Folch 2000] A. Folch, M. Toner, "Microengineering of cellular interactions". Annual Review of Biomedical Engineering, Vol.- 2, pp.227-256, 2000. 
Master's Thesis - Sarvesh Upadhyaya - McMaster University - Mechanical Engineering

[Freshly 1994] R. I. Freshney, "Culture of animal cells: Manual of basic technique", John Wiley and Sons, New York, 1994.

[Fu 1999] A. Fu, C. Spence, A. Scherer, F. Arnold, and S. Quake, "A microfabricated fluorescence-activated cell sorter", Nature Biotechnology, Vol.-17, pp.1109-1111, 1999.

[Fu 2003] P.F. Fu, S. Glover, R.K. King, C.I. Lee, M.R. Pretzer, M.K. Tomalia, "Polypropylene-polysiloxane block copolymers via hydrosilylation of monovinylidene capped isotactic polypropylene," ACS Polymer Preprint., Vol. 44, pp. 1014-1015, 2003.

[Fujji 2002] T. Fujji, "PDMS-based microfluidic devices for biomedical applications", Microelectronic Engineering Vol.-61,pp. 907-914, 2002.

[Gardener 2004] J. Gardner, S, Heffner, "Early stage drug discovery 2004", Kolarama Information, New York, 2004.

[Giordano 2001] B. C. Giordano, J. Ferrance, S. Swedberg, A. F. R. Huhmer, and J. P. Landers, "Polymerase chain reaction in polymeric microchips: DNA amplification in less than 240 seconds", Analytical Biochemistry, Vol.-291, pp.124-132, 2001.

[Giuliano 1997] K. A. Giuliano, R. L. DeBiasio, R. T. Dunlay, A. Gough, J. M. Volosky, J. Zock, G. N. Pavlakis and D. L. Taylor, "High-Content Screening: A New Approach to Easing Key Bottlenecks in the Drug Discovery Process", Biomolecular Screening Vol.-2, pp. 249-259, 1997. 
Master's Thesis - Sarvesh Upadhyaya - McMaster University - Mechanical Engineering

[Greenwald 1992] I. Greenwald, and G. M. Rublnf, "Making a difference: the role of cell-cell interactions in establishing separate identities for equivalent cells", Cell, Vol.-69, pp.271-281,1992.

[Grevensen 1993] P. Gravesen, J. Branebjerg and O. S. Jensen, "Microfluidics-a review", Journal of Mioromechanics Microengineering, Vol.-3, pp.168-182,1993.

[Guzman 2006] K. A. D. Guzmán, R. N. Karnik, J. S. Newman, A. Majumdar, "Spatially controlled microfluidics using low-voltage electrokinetics", Journal of Micro Electro Mechanical Systems, Vol.-15, pp. 237- 245, 2006.

[Harrison 1993] D. J. Harrison, K. Fluri, K. Seiler, Z. Fan, C. S. Effenhauser and A. Manz, "Micromachining a Miniaturized Capillary Electrophoresis-Based Chemical Analysis System on a Chip" Science, Vol. 261, pp. $895-897,1993$.

[Helgason 2005] C. D. Helgason, C. L. Miller, "Basic cell culture protocols", Humana Press, New Jersey, 2005.

[Hertzberg 2000] R. P. Hertzberg and A. J. Pope, "High-throughput screening: new technology for the 21st century", Current Opinion in Chemical Biology,Vol.-4 pp. 445 $451,2000$.

[Huang 1999] S. Huang, and D.E. Ingber, "The structural and mechanical complexity of cell-growth control" Nature Cell Biol. Vol.-1, pp.131-138, 1999. 
Master's Thesis - Sarvesh Upadhyaya - McMaster University - Mechanical Engineering

[Hughes 2001] I. Hughes, D. Hunter, "Techniques for analysis and purification in high throughput chemistry", Current Opinion in Chemical Biology, Vol.-5, pp. 243-247, 2001.

[Hung 2004] P. J. Hung, P. J. Lee, P. Sabounchi, R. Lin, L. P. Lee, "Continuous perfusion microfluidic cell culture array for high-throughput cell-based assays" Biotechnology and Bioengineering, Vol.-89, pp. 1-8, 2005.

[Huser 2007] J. Huser, "High-Throughput Screening in Drug Discovery" Wiley-VCH Verlag Gmbh, Germany, 2007.

[Inoue 2001] I. Inoue, Y. Wakamoto, H. Moriguchi, K. Okano, K. Yasuda, "On-chip culture system for observation of isolated individual cells", Lab Chip Vol.-1, pp. 50-55, 2001.

[Jeon 2002] N. L. Jeon, H. Baskaran, S. K. W. Dertinger, G. M. Whitesides, L. V. D. Water and M. Toner, "Neutrophil chemotaxis in linear and complex gradients of interleukin-8 formed in a microfabricated device", Nature Biotechnology, Vol.-20, pp. 826-830, 2002.

[Kaul 1985] A. Kual, B. D. Ratner, and M. B. Schway, "Toxicity of polyetherurathane surfaces", Transactions of Society of Biomaterials, Vol.-7, pp.193-198, 1985.

[Kemery 1998] P.J. Kemery, J.K. Steehler, P.W. Bohn, “Electric field mediated transport in nanometer diameter channels", Langmuir, Vol.-14, pp. 2884-2889, 1998. 
Master's Thesis - Sarvesh Upadhyaya - McMaster University - Mechanical Engineering

[Khandurina 2002] J. Khandurina, A. Guttman, "Microchip-based high-throughput screening analysis of combinatorial libraries", Current Opinion in Chemical Biology, Vol.-6, pp.359-366, 2002.

[Kirby 2004] B. J. Kirby, E F. Hasselbrink, "Zeta potential of microfluidic substrates: 1. Theory, experimental techniques, and effects on separations", Electrophoresis, Vol.-25, pp. 187-202, 2004.

[Koutny 1996] L. B. Koutny, D. Schmalzing, T. A. Taylor, and M. Fuchs, "Microchip Electrophoretic Immunoassay for Serum Cortisol", Analytical Chemistry, Vol.-68, pp.18$22,1996$.

[Kumar 1994] A. Kumar, H. A. Biebuyck, and G. M. Whitesides, "Patterning Self Assembled Monolayers: Applications inMaterials Science" Langmuir Vol.-10, pp. 1498$151,1994$.

[Kuo 2001] T.C. Kuo, L.A. Sloan, J.V. Sweedler, P.W. Bohn, "Manipulating molecular transport through nanoporous membranes by control of electrokinetic flow: effect of surface charge density and Debye length", Langmuir, Vol.-17, pp. 6298-6303, 2001.

[Kuo 2003] T. C. Kuo, D. M. Cannon, M. A. Shannon, P. W. Bohn and J. V. Sweedler, "Hybrid 3D nanofluidic /microfluidic devices using molecular gates" Sensors \& Actuators A, Vol.-102, pp. 223-233, 2003. 
Master's Thesis - Sarvesh Upadhyaya - McMaster University - Mechanical Engineering

[Lahaye 1991] M. Lahaye, C. Rochas, "Chemical structure and physico-chemical properties of agar.”, Hydrobiologia, Vol.-126, pp137-148, 1991.

[Lakowicz 2006] J. R. Lakowicz "Principles of Fluorescence Spectroscopy" Springer, New York, 2006.

[Laser 2004] D. J. Laser, J. G. Santiago, A review of micropumps, Journal of Micromechanics and Microengineering, Vol.14, pp.R35- R64, 2004.

[Leclerc 2006] E. Leclerc, B. Davida, L. Griscomb, B. Lepioufleb, T. Fujiic, P. Layrolled and C. Legallaisa, "Study of osteoblastic cells in a microfluidic environment", Biomaterials Vol.-27, pp.586-595, 2006.

[Lee 1995] K. Y. Lee, N. LaBianca, S. A. Rishton, S. Zolgharnain, J. D. Gelorme, J. Shaw, and T. H. P. Chang, "Micromachining applications of a high resolution ultra-thick photoresist", Journal of Vacuum Science \& Technology B: Microelectronics and Nanometer Structures, Vol.-13, pp. 3012-3016, 1995.

[Lee 2005] C. C. Lee, G. Sui, A. Elizarov, C. J. Shu, Y.S. Shin, A. N. Dooley, J. Huang, A. Daridon, P. W, D. Stout, H. C. Kolb, O. N. Witte, N. Satyamurthy, J. R. Heath, M. E. Phelps, S. R. Quake, H. R. Tseng, "Multistep synthesis of a radio labeled imaging probe using integrated microfluidics" Science Vol.310, pp.1793-1796, 2005. 
Master's Thesis - Sarvesh Upadhyaya - McMaster University - Mechanical Engineering

[Li 1997] P. Li, D. Harrison, "Transport, manipulation, and reaction of biological cells on-chip using electrokinetic effects', Analytical Chemistry, Vol.-69, pp.1564-1568, 1997.

[Lipinki 1997] C.A. Lipinski, F. Lombardo, B.W. Dominy, P.J. Feeney, "Experimental and computational approaches to estimate solubility and permeability in drug discovery and development settings", Advanced Drug Delivery Reviews, Vol.-23, pp.3-25, 1997.

[Liptrot 2002] C. Liptrot, "High content screening - from cells to data knowledge", Drug Discovery Today, Vol.-6, pp.832-834, 2002.

[Lorenz 1997] H Lorenz, M Despont, N Fahrni, N LaBianca, P Renaud and P Vettiger, "SU-8: a low-cost negative resist for MEMS" Journal of Micromechancis and Microengineering, Vol-7, pp.121-124, 1997.

[Macpherson 2002] J. V. Macpherson, C. E. Jones, A. L. Barker, and P. R. Unwin, "Electrochemical imaging of diffusion through single nanoscale pores", Analytical Chemistery, Vol.-74, pp.1841-1848, 2002.

[Madigan 2005] M. Madigan M and J. Martinko, "Brock Biology of microorganisms", Prentice Hall, 2005.

[Major 1998] J. Major, "Challenges and opportunities in high throughput screening: Implications for new technologies", Journal of Biomolecular Screening, Vol.-3, pp.13$17,1998$. 
Master's Thesis - Sarvesh Upadhyaya - McMaster University - Mechanical Engineering

[Manz 2004] A. Manz, C. S. Effenhauser, N. Burggraft, D. J.. Harrison, K. Seiler, and K. Flur, "Electroosmotic pumping and electrophoretic separations for miniaturized chemical analysis systems", Journal of Micromechancis and Microengineering, Vol.-4 pp. 257$265,1994$.

[Mata 2005] A. Mata, A. J. Fleischman, S. Roy, "Characterization of Polydimethylsiloxane (PDMS) Properties for Biomedical Micro/Nanosystems", Biomedical Microdevices, Vol.-7, pp.281-293, 2005.

[Mere 1999] L. Mere, T. Bennett, P. Coassin, P. England, B. Hamman, T. Rink, S. Zimmerman, P. Negulescu, "Miniaturized FRET assays and microfluidics: key components for ultra-high-throughput screening”, Drug Discovery Today, Vol.-4, pp.363-369, 1999.

[Miyake 1993] R. Miyake, T.S.J. Lammerink, M. Elwenspoek, J.H.J. Fluitman, “Micro mixer with fast diffusion" Proceedings of 6th IEEE International Workshop in MicroElectromechanical System, pp 248-53, 1993.

[Nguyen 2005] N.T. Nguyena and J. Wu, "Micromixers: A review", Journal of Micromechanics and Microengineering, Vol.-15, pp.R1-R16, 2005. 
Master's Thesis - Sarvesh Upadhyaya - McMaster University - Mechanical Engineering

[Obrien 1978] R. W . Obrien and L. R. White, "Electrophoretic Mobility of a Spherical Colloidal Particle", Journal of Chemical Society, Faraday Transactions. 2, Vol.-74, pp.1607-1626, 1978.

[Oh 2006] K. W. Oh and C. H. Ahn, "Microvalves: A review", Journal of Micromechanics and Microengineering, Vol.-16, pp.R13-R39, 2006.

[Pancrazio 1999] J. Pancrazio, J. Whelan, D. Borkholder, W. Ma, D. Stenger, "Development and application of cell-based biosensors", Annals Biomedical Engingeering, Vol.- 27, pp. 697-711, 1999.

[Park 2003] T.H. Park, M.L. Shuler. "Integration of cell culture and microfabrication technology", Biotechnology Progress, Vol.-19, pp. 243-253, 2003.

[Pihl 2005] J. Pihl, M. Karlsson and D. T.Chiu, "Microfluidics technology in drug discovery", Drug Discovery Today, Vol.-10, pp. 1377-1383, 2005.

[Pihl 2005b] J. Pihl, J. Sinclair, Karlsson and O. Orwar, "Microfluidics for cell based assays", Material Today, Vol.-8, pp. 46-51, 2005.

[Powers 2002] M.J. Powers, K. Domansky, M.R. Kaazempur-Mofrad, A. Kalezi, A. Capitano, A. Upadhyaya, P. Kurzawski, K.E. Wack, D.B. Stolz, R. Kamm, "A microfabricated array bioreactor for perfused 3D liver culture", Biotechnology Bioengineering, Vol.-78, pp. 257-269, 2002. 
Master's Thesis - Sarvesh Upadhyaya - McMaster University - Mechanical Engineering

[Raty 2001] S. Raty, J. A.Davis, D. J. Beebe, S. L. Rodriguez- Zas, M. B. Wheeler, "Culture in microchannels enhances in vitro embryonic development of preimplantation mouse embryos", Theriogenology Vol.55, pp.-241

[Reber 2001] N. Reber, A. Kuchel, R. Spohr, A. Wolf, M. Yoshida, "Transport properties of thermo-responsive ion track membranes", Journal of Membrane Science Vol.-193, pp.-49-58, 2001.

[Rees 1997] D A Rees, E. J. Welsh, "Secondary and tertiary structure of polysaccharides in solutions and gels", Angewandte Chemie International De English, Vol. 16 pp 214244, 1977.

[Rice 1965] C. L. Rice, R, Whitehead, "Electrokinetic flow in a narrow cylindrical capillary", Journal of Physical Chemistry, Vol.-69, pp.4017- 4029, 1965.

[Rostovtseva 1996] T.K. Rostovtseva, C.L. Bashford, G.M. Alder, G.N. Hill, C. McGiffert, P.Y. Apel, G. Lowe, C.A. Pasternak "Diffusion through narrow pores: movement of ions, water and nonelectrolytes through track-etched petp membranes", Journal of Membrane Biology, Vol-151, pp. 29-43, 1996.

[Shaw 1997] J. M. Shaw, J. D. Gelome, N. C. Labianca, W. E. Conley and S. J. Holmes, "Negative photoresists for optical lithography", IBM Journal of Research Development, Vol.- 41 ,pp. 81-95, 1997. 
Master's Thesis - Sarvesh Upadhyaya - McMaster University - Mechanical Engineering

[Sinclair 2002] J. Sinclair, J. Pihl, J. Olofsson, M. Karlsson, K. Jardemark, D. T. Chiu and O. Orwar, "A cell-based bar code reader for high-throughput screening of ion channel-ligand interactions", Analytical Chemistry, Vol.-74 pp. 6133-6138, 2002.

[Sinclair 2003] J. Sinclair, J. Olofsson, J. Pihl, and O. Orwar, "Stabilization of highresistance seals in patch-clamp recordings by laminar flow", Analytical Chemistry Vol.75 pp. 6718-6722, 2003.

[Smits 1990] J. G. Smits, "Piezoelectric micropump with 3 valves working peristaltically" Sensors Actuators A Vol.21, pp. 203-206, 1990.

[Streuli 1991] C. H. Streuli, N. Bailey, and M. J. Bissell, "Control of mammary epithelial differentiation: basement membrane induces tissue-specific gene expression in the absence of cell-cell interaction and morphological polarity", The Journal of Cell Biology, Vol.-115, pp.1383-1395, 1991.

[Sundberg 2000] S. A. Sundberg, "High throughput and ultra-high throughput screening: solution and cell based approaches", Current Opinion in Biotechnology, Vol.-11, pp. 47$53,2000$.

[Takayama 2001] S. Takayama, E. Ostuni, P. LeDue, K. Naruse, D. Ingber, G. M. Whitesides, "Laminar flows: subcellular positioning of small molecules", Nature Vol.411, pp.1016-1016, 2001. 
Master's Thesis - Sarvesh Upadhyaya - McMaster University - Mechanical Engineering

[Tampion 1987] J. Tampion, M. D. Tampion, "Immobilized cells: principles and applications", Cambridge University Press, Cambridge, 1997.

[Taylor 1997] T. B. Taylor, E. S. Winn-Deen, E. Picozza, T. M. Woudenberg and M. Albin, "Optimization of the performance of the polymerase chain reaction in siliconbased microstructures", Nucleic Acids Research, Vol.-25, pp. 3164-3168, 1997.

[Terry 1979] S. C. Terry, J. H. Jerman, and J. B. Angell, "A gas chromatographic air analyzer fabricated on a silicon wafer", IEEE Transactions, Electron Devices Vol-26, pp.1880-1886, 1979.

[Tran 2005] L. Tran, J. Farinas, L. R. Litrus, P. B. Conley, C. Muir, K. Munnelly, D. M. Sedlock, D. Diana and B. Cherbavaz, "Agonist-induced calcium response in single human platelets assayed in a microfluidic device", Analytical Biochemistry, Vol.-341 pp. $361-365,2005$.

[Trimmer 1996] W. Trimmer (Ed.), "Micromechanics and MEMS: Classic and Seminal Papers to 1990", IEEE Press, New York, 1996.

[Unger 2000] M. A. Unger, H. Chou, T. Thorsen, A. Scherer,S. R. Quake, "Monolithic microfabricated valves and pumps by multilayer soft lithography", Science Vol.-288, pp. 113-116, 2000. 
Master's Thesis - Sarvesh Upadhyaya - McMaster University - Mechanical Engineering

[Vo-Dinh 2001] T. Vo-Dinh, B. Cullum, D. Stokes, "Nanosensors and biochips: frontiers in biomolecular diagnostics", Sensors and Actuators B, Vol.-74, pp. 2-11, 2001.

[Walker 2002] G. M. Walker, M. S. Ozers, D. J. Beebe, "Insect cell culture in microfluidic channels", Biomedical Microdevices, Vol.4, pp. 161-166, 2002.

[Wang 1993] N. Wang, J.P. Butler, and D.E. Ingber "Mechanotransduction across the cell surface and through the cytoskeleton" Science Vol.-260, pp.1124-1127, 1993.

[Wautelet 2001] M. Wautelet, "Scaling laws in the macro-, micro- and nanoworlds", European Journal of Physics, Vol.-22, pp.601- 611, 2001.

[Welty 2000] J. R. Welty, C. E. Wicks, R. E. Wilson, G. Rorrer, "Fundamentals of Momentum, Heat, and Mass Transfer", John Wiley and Sons, Inc. New York, 2000.

[Whitesides 2001] G. M.Whitesides, E. Ostuni, S.Takayama, X. Jiang and D. E. Ingber, "Soft Lithography In Biology and Biochemistry", Annual Review Biomedical Engineering, Vol.-3, pp. 335-73, 2001.

[Whitesides 2002] G. M. Whitesides, J. C. Mcdonald "Poly dimethylsiloxane as a material for fabricating microfluidic devices", Accounts of Chemical Research, Vol.-35, pp.491-499, 2002. 
Master's Thesis - Sarvesh Upadhyaya - McMaster University - Mechanical Engineering

[Whitesides 2006] G. M. Whitesides, "The origins and the future of microfluidics", Nature, Vol.-442, pp. 368-373, 2006.

[Wieme 1965] R. J. Wieme, "Agar Gel Electrophoresis", Elsevier Publishing Company, New York, 1965.

[Xia 1997] Y. Xia and G. M. Whitesides, "Extending Microcontact printing as a microlithographic technique", Langmuir, Vol-13, pp.2059-2067, 1997.

[Xia 1998] Y. Xia and G. M. Whitesides, "Soft Lithography", Angewandte Chemie International Edition, Vol.-37, pp. 550-575, 1998.

[Xia 2007] Xuhua Xia, "Protein isoelectric point" in "Bioinformatics and the Cell", Springer USA 2007.

[Yanagisawa 1993] K. Yanagisawa, H. Kuwano and A. Tapo, "An electromagnetically driven microvalve" Proceeding Transducers pp. 102-105, 9.

[Zheng 2004] B. Zheng, J. D. Tice, L.S. Roach, and R. F. A. Ismagilov, "Droplet-based, composite PDMS/glass capillary microfluidic system for evaluating protein crystallization conditions by microbatch and vapor-diffusion methods with on-chip X-ray diffraction", Angewandte Chemie International Edition. Vol.-43, pp. 2508-2511, 2004. 
Master's Thesis - Sarvesh Upadhyaya - McMaster University - Mechanical Engineering

[Ziauddin 2000] J. Ziauddin and D. M. Sabatini, "Microarrays of cells expressing defined cDNAs", Nature, vol.-411, pp.107-110, 2000. 


\section{APPENDIXES}

\section{APPENDIX 1: SU8 MOLD FRABRICATAION}

1. Clean silicon with wafer water and acetone for two minutes.

2. Clean the wafer with HF for one minute.

3. Clean wafer again to remove any HF.

4. Put wafer in plasma oxidation machine. Power rating $\sim 50 \mathrm{~W}$ for 30 seconds.

5. Mount the wafer on spinner.

6. Pour little (1-2ml) amount of SU8 on wafer.

7. Spin the wafer at $500 \mathrm{rpm}$ for 5 seconds then continuous ramping to $2750 \mathrm{rpm}$ in another 5 seconds and then spin at constant speed for 30 seconds.

8. Pre-bake the SU coated at $65^{\circ} \mathrm{C}$ for 15 minutes.

10. Increase the pre-baking temperature to $95^{\circ} \mathrm{C}$ in 5 minutes and bake at constant temperature for $1 \mathrm{hr}$.

11. Mount the wafer on mask aligner.

12. Expose to Hg-365nm wavelength UV light for 60 seconds with $600 \mathrm{~mJ} / \mathrm{cm}^{2}$ energy density. 
Master's Thesis - Sarvesh Upadhyaya - McMaster University - Mechanical Engineering

12. Start post exposure bake at $65^{\circ} \mathrm{C}$ for 5 minutes.

13. Increase post exposure bake temperature to $95^{\circ} \mathrm{C}$ in 5 minutes and maintain constant temperature for 10 minutes.

14. Develop the SU-8 by submersing the wafer in SU-8 developer. Stir the solution every 5 minutes.

15. To check completion of development process, apply isopropyl alcohol on the wafer, a cloudy solution means incomplete development. Let the development continue till completion.

17. Dry the wafer the nitrogen gas. 


\section{APPENDIX 2: PDMS MICROCONTACT PRINTING}

1. Mix 1:10 base to curing agent of PDMS.

2. Pour enough of above liquid PDMS on a SU8 master to make $1 \mathrm{~mm}$ thick sheet.

3. Heat the SU8 at $60^{\circ} \mathrm{C}$ for 2 hours.

4. Carefully peel off the PDMS from SU8 masters using tweezers. This PDMS sheet will be used as stamp.

5. Mix $1 \mathrm{ml}$ of PDMS base with $3 \mathrm{ml}$ of curing agent using syringes.

6. Clean a silicon wafer with distilled water and acetone.

7. Mount the wafer on spinner. Pour little (1-2 ml) liquid PDMS on the wafer.

8. Start spinning by ramping from $0 \mathrm{rpm}$ to $10000 \mathrm{rpm}$ in 1 minute $(\sim 1000 \mathrm{rpm} / 6$ seconds).

9. Continue spinning at $10,000 \mathrm{rpm}$ for 4 minutes.

10. Carefully place the PDMS sheet stamp on the liquid PDMS coated silicon wafer. The face which contains the features would face down.

11. Use tweezers to carefully place PDMS sheet so that wafer touches all over the PDMS sheet. Care should be taken to avoid gap formation between PDMS stamp and silicon wafer.

12. Remove PDMS sheet from the wafer. 
Master's Thesis - Sarvesh Upadhyaya - McMaster University - Mechanical Engineering

13. Put the PDMS sheet on a clean surface and place nanoporous membrane on top of it.

14. Step 7-13 can be repeated for the same membrane to get desired patterning.

15. Cure PDMS sheet along with nanoporous membrane by heating at $60^{\circ} \mathrm{C}$ for 2 hours.

16. Peel off membrane from PDMS to get the membrane with patterning. 


\section{APPENDIX 3: DEVICE ASSMEBLY PROCESS}

1. Take all three individual layers (top PDMS layer, middle membrane, bottom PDMS layer) and clean them using Acetone if needed.

2. Mix $5 \mathrm{ml}$ of PDMS base with $0.5 \mathrm{ml}$ of curing agent using syringes.

3. Clean a silicon wafer with distilled water and acetone.

4. Mount the wafer on spinner. Pour little (1-2 ml) liquid PDMS on the wafer.

5. Start spinning by ramping from $0 \mathrm{rpm}$ to $10000 \mathrm{rpm}$ in 1 minute $(\sim 1000 \mathrm{rpm} / 6$ seconds).

6. Continue spinning at $10,000 \mathrm{rpm}$ for 4 minutes.

7. Put bottom PDMS layer on liquid PDMS coated silicon wafer. Use tweezers to carefully place PDMS sheet so that wafer touches all over the PDMS sheet. Care should be taken to avoid gap formation between PDMS stamp and silicon wafer.

8. Remove bottom PDMS layer from wafer.

9. Repeat steps 4-9 for top PDMS layer.

10. Place all three layers on top of each other, liquid PDMS sticking to top and bottom PDMS sheets act as glue.

11. Heat the assembly at $60^{\circ} \mathrm{C}$ for $2 \mathrm{~h}$. 
Master's Thesis - Sarvesh Upadhyaya - McMaster University - Mechanical Engineering

12. Heat $50 \mathrm{ml}$ of water (or other desired electrolyte e.g. phosphate buffer) up to 100 ${ }^{\circ} \mathrm{C}$.

13. Mix required amount of agar powder in boiling water. Boil for 2 minutes for agar to melt.

14. Take required amount of liquid gel using syringe.

15. Fill the liquid gel in the device and let it cool down. 


\section{APPENDIX 4: VISUALIZATION OF PROTEIN}

The visualization was done using SimplyBlue ${ }^{\mathrm{TM}}$ stain method of Nitrogen Inc. It involved following steps:

1. Wash the gel twice after protein pumping is done by distilled water. The washing is to be done by submerging the gel in distilled water for 1 minute.

2. Keep gel submerged in SafeBlue stain for 1 hour. The volume of stain should be 5 times the volume of gel.

3. Remove the gel and wash it again using distilled water as described in point 1.

4. Keep the gel submerged in lot of distilled water for 2 hours.

5. Repeat steps 3-4 if contrast between stained regions and background is not very clear.

The SimplyBlue ${ }^{\mathrm{TM}}$ stain dye, a proprietary solution of Invitrogen, bonds with protein molecules. A large amount of this dye on boding would give blue color to the area where protein is present. As this dye also bonds with gel (much lesser extent) de-staining steps (step 3 and 4 above) are needed to remove dye bonded with gel. 


\section{APPENDIX 5: VISUALIZATION OF DNA}

Ethidium Bromide (EtBr) is an intercalating agent commonly used to stain DNAs in molecular biology. After bonding to DNA, when exposed to ultraviolet light, EtBr will fluoresce with a red-orange color. Ethidium bromide is feared to be very strong mutagen, and may possibly be a carcinogen so special care should be taken in handling it. The protocol for DNA visualization using EtBr is given below.

1. Make $10 \mathrm{mg} / \mathrm{ml}$ stock solution of $\mathrm{EtBr}$ from form EtBr powder. This solution can directly be ordered from supplier (Sigma Aldrich). Directly ordering stock solution eliminates the need to work with power $\mathrm{EtBr}$ (this would reduce chances of $\mathrm{EtBr}$ exposure as stock solution is much diluter as compared to powder $\mathrm{EtBr}$ ).

2. Mix required amount of agar power in water (or TAE buffer) and boil it so that gel melts and dissolves.

3. Pour liquid gel in a smaller flask using syringe. Using syringe would eliminate small bubble formation.

4. Mix required amount of $\mathrm{EtBr}$ from stock solution to get $0.5 \mu \mathrm{g}$ of $\mathrm{EtBr}$ for per $\mathrm{ml}$ of agar gel.

5. Take EtBr mixed liquid form gel and fill into the device. Stay for some time so that gel solidifies.

6. Run the device and pump DNA. 
Master's Thesis - Sarvesh Upadhyaya - McMaster University - Mechanical Engineering

7. After pumping of DNA, remove the gel from the device and wash it using distilled water.

8. Put the gel sheet containing DNA in a dark chamber having provision for UV light (UV light is hazardous) illumination. Take picture using normal camera. 


\section{APPENDIX 6: MICROCHEM CORP. SU8-100 DATA SHEET}

Taken from manufacturer (MicroChem Corporation USA) data sheet.

\subsection{SU-8 Spinning Speed vs. Film Thickness}

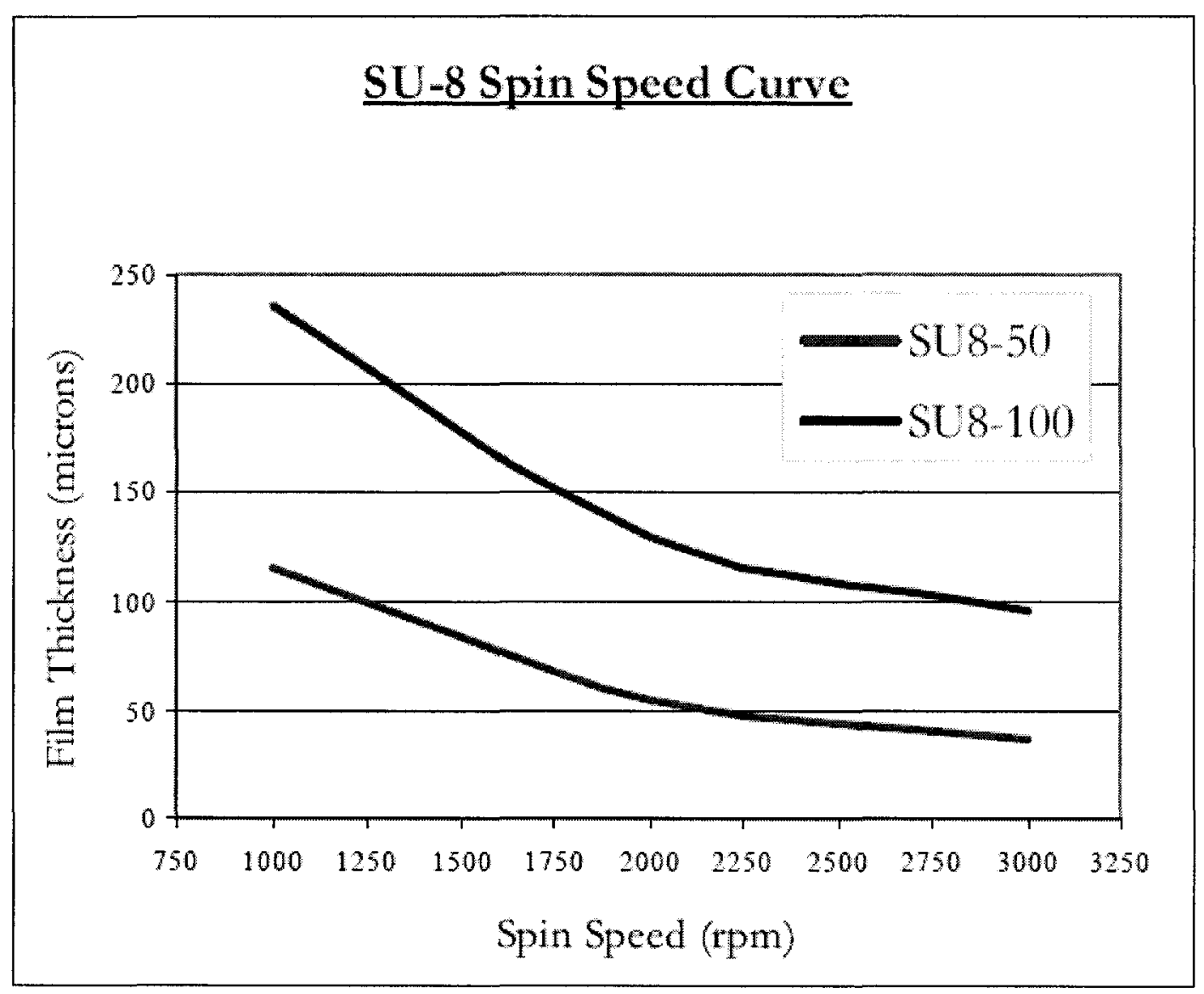


Master's Thesis - Sarvesh Upadhyaya - McMaster University - Mechanical Engineering

\subsection{SU-8 Film Thickness vs. Exposure Energy}

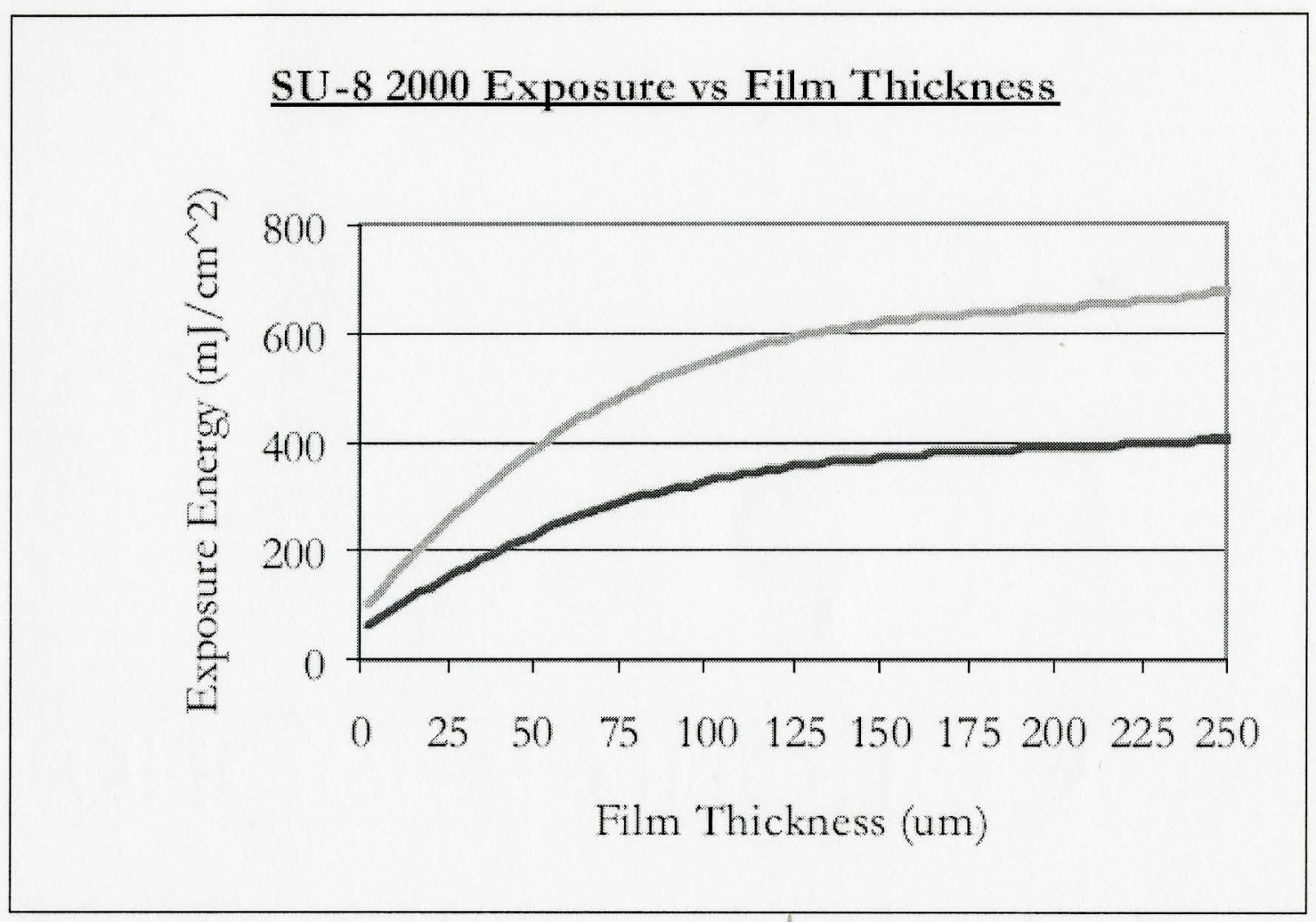




\section{APPENDIX 7: POLYCARBONATE MEMBRANE DATA SHEET}

(Source manufacturer data sheet provided by GE Osmonics).

\subsection{Product Characteristics}

\begin{tabular}{|l|l|}
\hline Sterilization & Gamma Irradiation or Ethylene Oxide (EtO) \\
\hline USP Class VI Testing & Passed \\
\hline Maximum Operating & $140^{\circ} \mathrm{C}\left(284^{\circ} \mathrm{F}\right)$ \\
Temperature & \\
\hline Sealing Compatibility & Ultrasonic, Heat, Radio Frequency and Insert Molding \\
\hline \hline Pore Size Range & 0.01 to $20 \mu \mathrm{m}$ \\
\hline \hline Autoclavable & Yes \\
\hline \hline Hydrophilic & Yes (Hydrophobic PVP-Free is also available) \\
\hline \hline pH & No \\
\hline \hline
\end{tabular}


Master's Thesis - Sarvesh Upadhyaya - McMaster University - Mechanical Engineering

\subsection{Performance Characteristics}

\begin{tabular}{|l|l|l|l|l|}
\hline $\begin{array}{l}\text { Pore } \\
(\boldsymbol{\mu m})\end{array}$ & $\begin{array}{l}\text { Pore } \\
\text { Density } \\
\left(\text { pores } / \mathrm{cm}^{2}\right)\end{array}$ & $\begin{array}{l}\text { Nominal } \\
\left(\mathrm{mg} / \mathrm{cm}^{2}\right)\end{array}$ & $\begin{array}{l}\text { Nominal } \\
\text { Thickness }\end{array}$ & $\begin{array}{l}\text { Min. Bubble } \\
\text { Point } \\
(\mathrm{psi})\end{array}$ \\
\hline $\mathbf{2 0}$ & $4 \times 10^{4}$ & 1.1 & 10 & 0.1 \\
\hline $\mathbf{1 4}$ & $5 \times 10^{4}$ & 0.6 & 6 & 0.2 \\
\hline $\mathbf{1 2}$ & $1 \times 10^{5}$ & 0.9 & 8 & 0.4 \\
\hline $\mathbf{1 0}$ & $1 \times 10^{5}$ & 1.1 & 10 & 0.5 \\
\hline $\mathbf{8}$ & $1 \times 10^{5}$ & 0.8 & 7 & 0.7 \\
\hline $\mathbf{5}$ & $4 \times 10^{5}$ & 1.1 & 10 & 1.2 \\
\hline $\mathbf{2}$ & $2 \times 10^{6}$ & 1.1 & 10 & 3 \\
\hline $\mathbf{0 . 8}$ & $3 \times 10^{7}$ & 0.9 & 9 & \\
\hline & $2 \times 10^{6}$ & 0.9 & 9 & \\
\hline 7 & 1.1 & 11 & 6 \\
\hline & & & & \\
\hline & & & & \\
\hline & & & & \\
\hline & & & & \\
\hline & & & & \\
\hline & & & & \\
\hline
\end{tabular}




\begin{tabular}{|l|l|l|l|l|}
\hline $\mathbf{0 . 6}$ & $3 \times 10^{7}$ & 1.0 & 9 & 9 \\
\hline $\mathbf{0 . 4}$ & $1 \times 10^{8}$ & 1.0 & 10 & 12 \\
\hline $\mathbf{0 . 2}$ & $3 \times 10^{8}$ & 1.1 & 10 & 20 \\
\hline $\mathbf{0 . 1}$ & $4 \times 10^{8}$ & 0.7 & 6 & 30 \\
\hline $\mathbf{0 . 0 8}$ & $4 \times 10^{8}$ & 0.7 & 6 & 38 \\
\hline $\mathbf{0 . 0 5}$ & $6 \times 10^{8}$ & 0.7 & 6 & 50 \\
\hline $\mathbf{0 . 0 3}$ & $6 \times 10^{8}$ & 0.7 & 6 & NA \\
\hline $\mathbf{0 . 0 1}$ & $6 \times 10^{8}$ & 0.7 & 6 & NA \\
\hline
\end{tabular}




\section{APPENDIX 8: BOVINE SERUM ALBUMIN PROPERTIES}

\section{SUPPLIER DATA SHEET}

(Sigma Aldrich)

SIGMA

ALBUMIN, BOVINE

Productintormation

CAS NUMBER: 9048-46-8

SYNONYMS: Bovine Serum Albumin: Bovine Plasma Albumin: BSA

STRUCTURE:

The molecular weight of BSA has frequently been cited as $66.120^{\prime}$ or $66,267^{2}$, but it was revised in 1990 to $66,430^{3}$. All three values are based on amino acid sequence information avallable at the time of publication.

BSA is a single polypeptide chain consisting of about 583 amino acid residues and no carbohydrates. At pH 5-7 it contains 17 intrachain disulfide bridges and 1 sulfhydryl group.

\section{PHYSICAL PROPERTIES}

Appearance: Powder - White to light tan ${ }^{4}$

Solutions - Clear to slightly hazy and amber

pl in Water at 25EC: Endogenous Material ${ }^{3.6}$ - 4.7;4.9;

Falty Acid Depleted ${ }^{3}+5.3$

$\mathrm{pH}$ of $1 \%$ Solution: ${ }^{1.4} 5.2-7$;

Optical Rotation: ${ }^{1.9}[(c)]_{258}-61 \mathrm{E} ;[\alpha]_{264:-63 \mathrm{E}}$

Stokes Radius $\left(r_{3}\right): 3.48 \mathrm{~mm}$

Sedimentation constant ${ }^{1} S_{20} \times 10^{13}$

Diffusion constant ${ }^{\prime}, 0, x^{2}$

Parlial specific volume, $v_{z}$

Intrinsic viscosity, ${ }^{\prime}$ '

Frictional ratio, $f / f_{0}$,

Overall dimensions, $A$

Refractive indexincrement' $(578 \mathrm{~nm}) \times 10^{-3}$

Optical absorbance, 'A j,

Mean residue rotation. ${ }^{\prime}[m]_{233}$

Mean residue ellipticity

Estimated $\alpha$-helix. \%

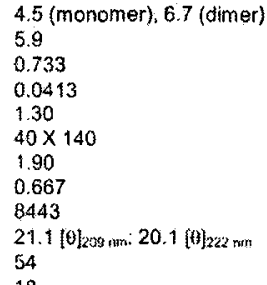

1.90

0.667

8443

$21.1[0]_{2 x s}$ man: $\left.20.1[0]\right]_{222 \mathrm{~nm}}$

54

Estimated $\beta$-form, \%

STABILITY / STORAGE AS SUPPLIED;

If stored at 2-81:C, BSA powders and BSA solutions offered by Sigma are stable for a minimum of 2.5 years. 
Master's Thesis - Sarvesh Upadhyaya - McMaster University - Mechanical Engineering

\section{ALBUMIN, BOVINE}

SOLUBILITY / SOLUTION STABILITY:

Albumins are readily soluble in water and can only be precipitated by high concentrations of neutral salts such as ammonium sulfate. Sigma tests the solubility of powdered BSA in deionized water at

$40 \mathrm{mg} / \mathrm{mL}$ and obtains clear to very slightly hazy, faint yellow solutions. The solution stability of BSA is very good (especially if the solutions are stored as frozen aliquots). In fact, albumins are frequently used as stabilizers for other solubilized proteins (e.g., labile enzymes). However, albumin is readily coagulated by heat. "When heated to $50 \mathrm{EC}$ or above, albumin quite rapidly forms hydrophobic aggregates which do not revert to monomers upon cooling. At somewhat lower temperatures aggregation is also expected to occur, but at relatively slower rates.

\section{METHOD OF PREPARATION:}

A. HISTORY: ${ }^{1.4}$ Albumin is relatively simple to isolate and purify. One of the first methods of isolation involved extensive dialysis of serum against water; this process removed most globulins. A second procedure took advantage of the good solubility of albumin at low to moderate ammonium sulfate concentrations, and effected precipitation by lowering the $\mathrm{pH}$. Electrophoretic isolation was also employed, as was affinity chromatography. None of these methods were applicable to large scale production.

B. INITIAL ISOLATION: Intitial isolation is by Heat Treatment or by Alcohol precipitation. Most commercial preparations are now prepared by Alcohol Precipitation a method developed by $\mathrm{E} . J$. Cohn and his associates in the 1940 's ("Fraction $V$ " yields albumin with a purity of about $96 \%$ ) or by Heat Treatment.

C. FURTHER PURIFICATION: ${ }^{\text {.4 }}$ Additional removal of impurities can be accomplished by crystallization (a procedure which yields $399 \%$ pure albumin), preparative electrophoresis, ion exchange chromatography, affinity chromatography (e.g. ConA-agarose removes glycopfoteins), heat treatment (removes globulins). low pH treatment, charcoal treatment, organic solvent precipitation (i.e., isooctane), and low temperature treatment. ${ }^{13}$ Charcoal treatment and organic solvent precipitation remove fatty acios.'

\section{PRODUCT DESCRIPTION / USAGE: ${ }^{14}$}

Albumins are a group of acidic proteins which occur plentifully in the body fluids and tissues of mammals and in some plant seeds. Unlike globulins, albumins have comparatively low molecular weights, are soluble in water, are easily crystalized, and contain an excess of acidic amino acids. Serum and plasma albumin is carbohydrate-free and comprises $55-62 \%$ of the protein present. 
Master's Thesis - Sarvesh Upadhyaya - McMaster University - Mechanical Engineering

\section{ALBUMIN, BOVINE}

PRODUCT DESCRIPTION / USAGE: (continued)

Albumin binds water, $\mathrm{Ca}^{2+}$. $\mathrm{Na}^{*}$, and $\mathrm{K}^{*}$. Due to a hydrophobic cleft, albumin binds fatty acids, bilirubin, hormones and drugs. The main biological function of albumin is to regulate the colloidal osmotic pressure of blood. Human and bovine albumins contain 16\% nitrogen and are often used as standards in protein calibration studies. Albumin is used to solubilize lipids, and is atso used as a blocking agent in Western blots or ELISA applications. Globulin free albumins are suitable for use in applications where no other proteins should be present (e.g., electrophoresis).

\section{CHOOSING A PRODUCT:}

Please refer to the table below for a complete description of each product. Based on customer imput literature reports and Sigma's own use, the following table lists product numbers which have successfully been used for specific applications. The list is not comprehensive, and product numbers not listed may often be substituted. 
ALBUMIN, BOVINE

\begin{tabular}{|c|c|}
\hline APPLICATION & PRODUCT NUMBER(S) \\
\hline Antibody purification & A2058 \\
\hline Binding and transport studies & $\begin{array}{l}\mathrm{A} 4378, \mathrm{~A} 7030, \mathrm{~A} 0281, \mathrm{~A} 3675 \\
\mathrm{~A} 3902, \mathrm{~A} 6003\end{array}$ \\
\hline Blood banking reagents & $\begin{array}{l}\text { A2153, A4503, A7888, A3294, } \\
\text { A3912, A7906, A7030 }\end{array}$ \\
\hline Culture media (microbial) & $\begin{array}{l}\mathrm{A} 2153, \mathrm{~A} 4503, \mathrm{~A} 3294, \mathrm{~A} 3912 . \\
\mathrm{A} 7906, \mathrm{~A} 9430, \mathrm{~A} 7638, \mathrm{~A} 6003\end{array}$ \\
\hline Cell culture (general) & A8806, A9418 \\
\hline Electrophoresis (M.W. standard) & A7517 \\
\hline ELISA (blocking reagent) & $\begin{array}{l}\text { A2153, A4503, A4378, A7030, } \\
\text { A9430, A3902 }\end{array}$ \\
\hline ELISA (non-specific binding) & A3294 \\
\hline Enzyme systems & $\begin{array}{l}\text { A2153, A4503, A7888, A3294, } \\
\text { A3912, A4378, A7906, A7030, } \\
\text { A9430, A7638, A3675 }\end{array}$ \\
\hline Hapten carrier & $\mathrm{A} 7030, \mathrm{~A} 6003$ \\
\hline Immunocytochemistry & A9647. A7906, A6793 \\
\hline Immunohematology & $\begin{array}{l}\text { A2153, A4503, A7888, A3294, } \\
\text { A3912, A4378, A7906. A7030, } \\
\text { A0281, A6003 }\end{array}$ \\
\hline Mitogenic assays & A2058 \\
\hline Molecular biology & 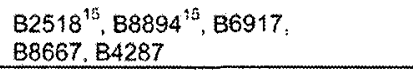 \\
\hline Protein base or filler & $\begin{array}{l}\text { A2153, A4503, A3912, A4378, } \\
\text { A7906, A7030 }\end{array}$ \\
\hline Protein supplement (controls) & $\begin{array}{l}\text { A2153, A4503, A4378, A7906, } \\
\text { A7030, A3675 }\end{array}$ \\
\hline Protein standard (MW., amino acids, nitrogen) & A2153,A4503, A4378, A7030 \\
\hline RIA systems & $\begin{array}{l}\text { A7888, A4378, A7030, A3675, } \\
\text { A3902 }\end{array}$ \\
\hline Serology & $\begin{array}{l}\text { A4503, A3912, A4378, A7906, } \\
\text { A7030, A9430, A3675 }\end{array}$ \\
\hline
\end{tabular}


Master's Thesis - Sarvesh Upadhyaya - McMaster University - Mechanical Engineering

\section{ALBUMIN, BOVINE}

\section{REFERENCES:}

1. The Plasma Proteins: Structure, Function and Genetic Control, 2nd ed., Frank W. Putnam, ed.

Vol. 1. p. 141, 147, Academic Press, New York (1975).

2. Reed, R.G. et al., Biochem. J., 191, 867 (1980)

3. Hirayama. K., BBRC. 173(2). 639 (1990)

4. Sigma data.

5. Dawson, R.M.C. et al., Data for Biochemical Research, 3rd ed, p. 381, Clarendon Press. Oxtord (1993).

6. Malamud, D. and Drysdale, J.W.. Anat. Biochem., 86, 620 (1978),

7. Righetti, P.G. and Caravaggio. T., J Chromatog. 127, 1 (1976)

8. Steinhardt, J. et al., Biochem., 10(22), $630(1971)$.

9. CRC Handbook of Biochemistry: Selected Data for Molecular Biology. H.A. Sober, ed. p. C.56, The Chemical Rubber Company, Cleveland (1968)

10. Axelsson, I., J. Chromatog. 152, 21 (1978).

11. Lewis, Sr., R.J. Hawley's Condensed Chemical Dictionary, 12th ed., p. 30. Van Nostrand Reinhold Co. New York (1993).

12. Cohn, E.J. et al., J. Am. Chem. Soc., 68, 459(1946).

13. Saifer, A. and Goldman, L. J. Lipid Res., 2(3), 268 (1961).

14. Scott, T. and Eagleson, M., Concise Encyciopedia: Biochemistry, 2nd ed., pp. 19-20, Walter de Gruyter. New York (1988).

15. These products are acetylated to inactivate nucleases commonly found in BSA, and are thus not listed in the table of unmodified BSA's on pp. 4-9 of this data sheet. Since tyrosines in the BSA are also derivatized, these preparations are not recommended for use as protein standards. 